\title{
CityNet: A Deep Learning Model for Trip Demand Prediction
}

\author{
By \\ Ahmad Seet \\ A thesis submitted to \\ the Faculty of Graduate Studies and Research \\ in partial fulfilment of \\ the requirements for the degree of \\ Master of Computer Science \\ Ottawa-Carleton Institute for Computer Science \\ School of Computer Science \\ Carleton University \\ Ottawa, Ontario
}

Date of submission March 15, 2021

(C) Copyright

2021, Ahmad Seet 


\section{Abstract}

Trip demand prediction is an integral part of intelligent transportation systems. It is concerned with estimating future trip demand based on past observations. An accurate forecasting model can help with the efficient reallocation of vehicle resources to better meet travel demands, which can benefit many transportation services. The prediction process is a challenging task due to the complex and dynamic spatio-temporal correlations of trip data. Recent advances in deep learning-based methods have inspired researchers to apply them to traffic forecasting tasks. Some of these methods use Convolution Neural Networks to model the spatio-temporal dependencies in trip data by representing trip data as $2 \mathrm{D}$ grids. Other methods utilize the natural graph structure of transportation networks and apply Graph Convolution Networks to model the propagation effects between graph nodes. However, these techniques impose certain spatial constraints on the data that do not represent real-world conditions or do not account for a changing and expanding transportation network structure. To address these limitations, we develop a method, entitled CityNet, that learns the demographic, socio-economic and land-use features of city regions to perform trip demand prediction. Specifically, our model first predicts the number of incoming and outgoing trips to all city regions and then utilizes these values to estimate the trip demand between region pairs. The experimental results show that our model achieves a minimum of $56 \%$ improvement in error rate compared to several time-series forecasting techniques and neural network methods, as well as a $15 \%$ improvement compared to two variants of our proposed model. 


\section{Acknowledgements}

I would like to thank my thesis supervisor, Professor Doron Nussbaum, for his advice, constructive feedback, and guidance through the preparation of this thesis.

I'm grateful to my wife and kids for their unwavering support and encouragement during my graduate studies at Carleton University. And I owe my parents a debt of gratitude for their patience and financial support over the years. 


\section{Contents}

$\begin{array}{ll}\text { Abstract } & \text { ii }\end{array}$

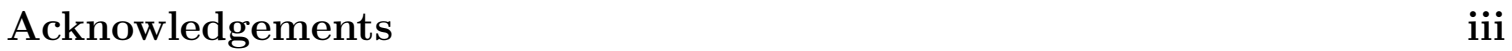

1 Introduction 1

1.1 Motivation . . . . . . . . . . . . . . . . . 1

1.2 Problem Definition . . . . . . . . . . . . . . . 3

1.3 Contribution Summary . . . . . . . . . . . . . . 5

1.4 Thesis Organization . . . . . . . . . . . . . . . 6

2 Literature Review $\quad 7$

2.1 Traditional Methods . . . . . . . . . . . . . . . . 8

2.1.1 Classical statistical models . . . . . . . . . . . . . . 8

2.1.2 Machine Learning Methods . . . . . . . . . . . . . 10

2.1.2.1 Bayesian Networks . . . . . . . . . . . . . 10

2.1.2.2 Support Vector Machines . . . . . . . . . . . 12

2.2 Deep Learning-based Methods . . . . . . . . . . . . . . . . . . 13

2.2 .1 Fully Connected Neural Network _. . . . . . . . . . . 13

2.2 .2 Convolution Neural Network . . . . . . . . . . . . . . 15

2.2.2.1 Research work that applies CNN for traffic forecasting 16

2.2 .3 Recurrent Neural Network . . . . . . . . . . . . . . . . 18

2.2.3.1 Research work that applies RNN for traffic forecasting 19

2.2.4 Graph Convolution Network . . . . . . . . . . . . . . . 21

2.2.4.1 Research work that applies GCN for traffic forecasting 22 
2.2.5 Attention Mechanism . . . . . . . . . . . . . . . 25

2.2.5.1 Research work that applies GAT for traffic forecasting 26

3 Methodology \& Implementation $\quad 29$

3.1 Preliminaries . . . . . . . . . . . . . . . . . . . . . . . . . . . . . 29

3.1 .1 City Representation . . . . . . . . . . . . . . . 30

3.2 Trip Demand Prediction Problem . . . . . . . . . . . . . . 31

3.2 .1 Problem Definition . . . . . . . . . . . . . . . . . 32

3.3 CityNet Model . . . . . . . . . . . . . . . . . . . . . . . . . 33

3.3 .1 Node Submodel . . . . . . . . . . . . . . . . . . . . . . . . . 34

3.3.1.1 Node Feature Preparation . . . . . . . . . . . . 34

3.3.1.2 Node State Computation . . . . . . . . . . . . 37

3.3.1.3 Localized Prediction \& Updating Node Attributes . . 39

$3.3 .2 \quad$ Edge Submodel . . . . . . . . . . . . . . . . . . . . . . . . . 39

3.3.2.1 Edge Feature Preparation . . . . . . . . . . . . 41

3.3.2.2 Trip Demand Prediction . . . . . . . . . . . . . 42

3.3.3 Learning Algorithm . . . . . . . . . . . . . . . . . . . . 45

3.3.3.1 Training the Node Submodel . . . . . . . . . . . 46

3.3.3.2 Training the Edge Submodel . . . . . . . . . . . 48

4 Experimental Results and Analysis $\quad 50$

4.1 Data Description \& Preparation . . . . . . . . . . . . . 50

4.1 .1 City Partitioning . . . . . . . . . . . . . . 52

4.1.1.1 Selection Criteria . . . . . . . . . . . . 52

4.1.1.2 Land-Use Classification . . . . . . . . . . . . 56

4.1.1.3 Region Selection . . . . . . . . . . . . 58

4.1 .2 Trip Records Dataset . . . . . . . . . . . . . . . . 58

4.1 .3 Node Attributes . . . . . . . . . . . . . . . . . . . . . 60

4.1 .4 Edge Attributes . . . . . . . . . . . . . . . . 60

$4.1 .5 \quad$ Global Attributes . . . . . . . . . . . . . . . . . . 61

4.1.6 Preparing The City Observation Set _. . . . . . . . . 61

4.2 Data Analysis . . . . . . . . . . . . . . . . . . . 64 
4.3 Experiments . . . . . . . . . . . . . . . . . . 67

4.3 .1 Comparison Methods . . . . . . . . . . . . . . . . 68

4.3 .2 Preprocessing . . . . . . . . . . . . . . . . 70

4.3 .3 Evaluation Metrics . . . . . . . . . . . . . . . . 71

4.3 .4 Hyperparameters . . . . . . . . . . . . . . . . . . . 72

4.3.5 Experimental Results . . . . . . . . . . . . . . . . . . 73

4.3.5.1 Performance Comparison with Baseline Methods . . 75

4.3.5.2 Performance Comparison with CityNet-Variants . . 77

4.3.5.3 Performance Comparison with Alternative (Untested) Models . . . . . . . . . . . . . . . . . . 79

4.3.5.4 Effect of Different Components on Model Performance 81

4.3.5.5 Effect of $\omega$ Size on the Node Submodel's Performance 82

4.3.5.6 Effect of $\omega$ Size on the Edge Submodel's Performance 84

4.3.5.7 Node Submodel's Performance Results per Land-Use Category . . . . . . . . . . . . . . . . 85

4.3.5.8 Edge Submodel's Performance Results per Land-Use Category . . . . . . . . . . . . . . . 9 90

5 Conclusions $\quad 95$

5.1 Benefits and Limitations . . . . . . . . . . . . . . . . 96

5.2 Future Work . . . . . . . . . . . . . . . . . . 98

$\begin{array}{ll}\text { Bibliography } & 100\end{array}$

A Land-Use Labeling Heuristic $\quad 113$ 


\section{List of Tables}

2.1 Summery of the traffic forecasting techniques discussed in Section 2.1 8

2.2 Summery of the traffic forecasting techniques discussed in Section $2.2 \quad 14$

3.1 General notations and symbols used in this chapter . . . . . . . . . 35

4.1 Time segments used for data collection and processing. . . . . . . . . 51

4.2 Illustration and description of four cartographic boundary files. . . . . 53

4.3 Train and test split by land-use category . . . . . . . . . . . . . 71

4.4 Experimental Results . . . . . . . . . . . . . . . . 74

4.5 Evaluation results for the alternative models as found on their respective papers. . . . . . . . . . . . . . . . . 80

4.6 Experimental model configurations. The convolution operation (conv) takes 2 parameters, kernel size, denoted by $\mathrm{k}$, and a stride dimension, denoted by s. . . . . . . . . . . . . . . . . . . 81

4.7 Evaluation results for the different model configurations. . . . . . . . 82 


\section{List of Figures}

2.1 Example of traffic data as a 2D grid (raster image) used for convolution (partial image taken from [1]) . . . . . . . . . . . . . . 15

2.2 Illustration of a stack of (a) causal convolution, and (b) dilated causal

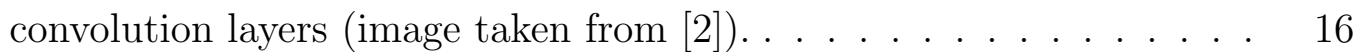

2.3 ST-ResNet architecture, where Conv is Convolution, ResUnit is Residual Unit, and FC is Fully-connected (image taken from [1]) . . . . . . 17

2.4 DMVST-Net architecture, where Conv is Convolution, FC is FullyConnected, Loss is the loss function, and Embed is feature embedding

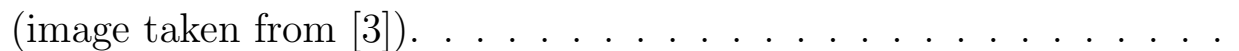

2.5 An illustration of the ST-GCN architecture, where $V^{t}$ is a finite set of vertices corresponding to traffic observations and $W$ denotes the weighted adjacency matrix of the graph (image taken from [4]). . . .

2.6 An illustration of the ST-MetaNet architecture, where $X_{t}$ is a vector corresponding to traffic observations and $\hat{Y}_{t}$ an output vector corresponding to traffic predictions at time $t$, and $H_{R N N}$ and $H_{M e t a-R N N}$ denote the hidden states (image taken from [5]) . . . . . . . . .

2.7 An example of Meta-GAT that calculates the impact to the red node from its neighborhoods along edges (image taken from [5]). . . . . .

3.1 A depiction of our city representation with associated attributes. . . . 
3.2 Illustration of the node feature preparation step. The process extracts the trip demand related to the selected node, illustrated in blue, and assigns them to the corresponding edges. Note, edges with 0 trip value are dotted out to signify that they are ignored during computation. The process then computes the flow $w^{+}$and flow $^{-}$for the selected node, and then constructs the feature vector. All values depicted in the figure are an example. . . . . . . . . . . . . . . . 36

3.3 GRU Cell Architecture . . . . . . . . . . . . . . . . . . . . . 37

3.4 Illustration of the full node submodel neural network, where output_1 is the predicted incoming trip value and output_2 is the predicted outgoing trip value. . . . . . . . . . . . . . . .

3.5 Edge Feature Preparation: The function propagates the node representation vectors to the selected edge, illustrated in blue. It then outputs the feature matrix by concatenating the edge attributes and global attributes to the result of the aggregation. All values depicted in the figure are an example. . . . . . . . . . . . . . . . . 41

3.6 Illustration of CNN operations in our network. The $n_{1}, n_{2}$ and $n_{3}$ are the number of kernels used for each respective layer. . . . . . . . . . 44

3.7 An illustration of all the layers in the CNN model. The output is the predicted trip demand. . . . . . . . . . . . . . . . . .

4.1 Trip volume per hour of the day . . . . . . . . . . . . . . . . 52

4.2 Statistical information regarding city regions and the trips within them. 54

4.3 Number of trip requests per region. The red star indicates the mean

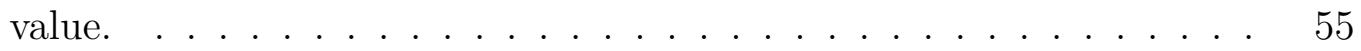

4.4 Percentage of regions with 0 trip requests . . . . . . . . 55

4.5 Illustration of a spatial join operation between Census tracts and ZoLa geometries, where $\oplus$, here, is the spatial join operand. For clarity, we only show one landuse type per polygon. The resulting geometries are Census Tracts with 1-or-more land-use information. . . . . . . . 57

4.6 Illustration of the final region selection in red. . . . . . . . . 58 
4.7 Preparing City Observations Flowchart . . . . . . . . . . . .

4.8 Correlation between total population per region and average trip requests per time interval, and marked by general land-use type. . . . .

4.9 Median household income in relation to average number of trip requests per time interval, and marked by general land-use type. . . . . . . . .

4.10 Average Trip count with respect to the $\%$ of population with bachelor or higher degree, and marked by general land-use type. . . . . . . . . 66

4.11 Box plot illustration of trip count by land-use type. The plots are grouped by trip volume level. The red star indicates the mean value. 66

4.12 Bar charts illustrating the evaluation results. . . . . . . . . . . . 74

4.13 Comparison between the actual trip demand values for four separate regions (labeled by land-use type) and their respective predicted values for XGBoost, WnD, GNN and CityNet-w36 models . . . . . . . . . . 76

4.14 Comparison between MSLE values for XGBoost, WnD, GNN and CityNet- $\omega 36$ models. . . . . . . . . . . . . . . . . . . . 77

4.15 Comparison between the actual trip demand values for four separate regions (labeled by land-use type) and their respective predicted values for CityNet-0, CityNet-MLP and CityNet- $\omega 36$ models . . . . . . . . . 78

4.16 Comparison between MSLE values for CityNet-0, CityNet-MLP, and CityNet- $\omega 36$ models. . . . . . . . . . . . . . . . . . .

4.17 Node submodel validation results during training with respect to varying sequential and cross-section $\omega$ values. . . . . . . . . . . .

4.18 Evaluation metric results for varying sequential and cross-section $\omega$ values. . . . . . . . . . . . . . . . . . .

4.19 Edge submodel validation results during training with respect to varying lengthwise and cross-section $\omega$ values. . . . . . . . . . . . . 84

4.20 Evaluation results for varying sequential and cross-section $\omega$ values. . $\quad 85$

4.21 The average and standard deviation of outgoing trip requests by region land-use category. . . . . . . . . . . . . . . . 86

4.22 Evaluation results per region land-use category. . . . . . . . . . . 86 
4.23 Comparison between the predicted outgoing trip requests and it respective actual values for an Airport region. . . . . . . . . . . . . . 87

4.24 RMSE results for predicted trip demand per individual CommercialLarge region. . . . . . . . . . . . . . . . . .

4.25 Comparison between the predicted total number of trip requests and its respective actual values for region "1225076148348" . . . . . . . . 88

4.26 RMSE rate for outgoing trip request predictions split by land-use type and colored by time interval (Airport regions were removed due to high error values). . . . . . . . . . . . . . . . . .

4.27 Comparison between the predicted outgoing trip requests and its respective actual values for two randomly selected regions. . . . . . . . 90

4.28 Average, standard deviation, and $99^{\text {th }}$ Percentile of trip demand by land-use category. . . . . . . . . . . . . . . . . . . . 91

4.29 Evaluation results by origin region land-use category. . . . . . . . . . 92

4.30 Evaluation results by origin region land-use category and by destination region land-use category. . . . . . . . . . . . . . . . .

4.31 Comparison between the predicted trip demand and its respective actual values for four randomly selected region pairs by land-use type. Note, we use green dots to indicate over and under estimation for specific time intervals. We also note that we use bar charts since we are depicting separate instances of travel not continues time steps. . . . .

4.32 Total number of records used by land-use category during the training of the CityNet model. . . . . . . . . . . . . . . . . . . . 


\section{Chapter 1}

\section{Introduction}

In this thesis, we propose a novel deep learning-based method for trip demand prediction. This chapter provides our motivation to conduct this research in Section 1.1, followed by the specific problem we manage in this work in Section 1.2. In Section 1.3 we summarize our thesis contributions, and lastly in Section 1.4 we outline the thesis structure.

\subsection{Motivation}

Trip prediction, or better known as traffic forecasting in many areas of transportation research, is one of the core components of intelligent transportation systems (ITS). It has been a topic of interest since 1979 [6] and is concerned with estimating traffic values for a short length of time in the future based on current and historical observations. Traffic involves one or multiple travel characteristics, which are mainly 
speed, travel-time, demand, and flow [7]. Estimating traffic values is a challenging problem affected by complex and dynamic spatial correlations, long-term temporal dependency, non-stationarity, and heterogeneity. In addition, traffic on a road network can be influenced by external factors, such as road conditions (road construction, congestion, and vehicle collision), weather conditions, regional events, amongst other factors [8]. The forecasting process also relies on the availability and completeness of historical traffic data collected from various sources, such as traffic sensors (loop detectors) on freeways or motorways, traffic data collected by transportation agencies, and trajectory data collected by global positioning systems (GPS) and cell towers [9].

Many real-world services and applications benefit from short-term trip forecasts. For example, traffic control and management systems can benefit from estimating the incoming and outgoing trip demand by controlling traffic flow and avoiding congested areas. Additionally, the dispatch systems of a taxi, ride-hailing, and ride-sharing companies (such as Uber, DiDi, and Lyft), can also benefit from estimating the incoming and outgoing demand by reallocating idle fleet vehicles in advance to anticipate and service upcoming demand. In doing so, passengers experience reduced wait times, vehicle service drivers experience decreased downtime, and the service providers profit from better utilization of vehicle resources and higher response rates.

Moreover, estimating accurate trip pickup and drop-off locations can greatly help ride-sharing companies provide an optimized service. Enroute vehicles (i.e., with passengers) can be intelligently rerouted to pass through areas with possible trip requests that share a common destination with onboard passengers. An optimized ride-sharing procedure with minimal overhead due to timely detours can highly encourage passengers to share rides. Therefore, an efficient and accurate trip demand forecasting 
system that can account for the complex spatio-temporal dependencies of trip data is significantly in-demand.

\subsection{Problem Definition}

Modeling the spatio-temporal correlations of traffic data has been the objective of countless traffic forecasting studies. In recent years, data-driven approaches have lead the research owing to a large amount of collected traffic information. These methods rely on deep neural network architectures to capture the complex spatial correlations and temporal dependencies in the data, and learn emerging patterns. Some of these studies model the traffic data as two-dimensional grids $([10,3,1,11])$ and use Convolutional Neural Networks to find near and distant spatial dependencies between regions of a city across time. However, a significant drawback of these studies is that they impose strong spatial constraints on the data and a specific degree of granularity that cannot accurately represent many real-world situations.

Other studies $([12,13,14,5,4])$ utilize the natural graph structure of a city's transportation network and use Graph Convolutional Networks to model the propagation effect of traffic flow on neighboring locations by unrolling static graphs over time, with nodes representing traffic at specific locations and edges representing the interaction and relation between traffic at different locations. However, a significant drawback of these studies is that they rely on a key assumption that the adjacency matrix used to represent the graph is strictly static and does not change over time. Yet, transportation networks are strongly affected by external conditions, unpredictable accidents, and unforeseen construction work that can render a node in the input graph 
inaccessible, which indicates that transportation networks are time-variant and thus cannot be reliably modeled by a static adjacency matrix. Additionally, for many developing and expanding cities, where road segments are regularly introduced into the transportation network, a static adjacency matrix cannot efficiently account for these inclusions. Nonetheless, dynamic representations of the transportation network have been proposed by [15] and [16]. However, these models still rely on a form of adjacency matrix that is not readily available and limited by the number of nodes it can represent.

To overcome the aforementioned limitations, we propose a deep learning framework, titled CityNet, for trip demand forecasting that is independent of fixed locations but can capture underlining relationships between city regions. The main intuition is to learn the impact of demographic and socio-economic information (non-spatial features) pertaining to city regions on trip demand and understand human movement patterns by classifying city regions with land-use categories. For example, people travel from residential to commercial areas in the morning for work and vice versa in the afternoon, which indicates the spatial and temporal relations of human travel behavior.

Our framework represents a city as a dynamic graph structure to alleviate spatial constraints, where nodes represent city regions and edges signify traffic flow between them. The approach relies on a two-step process:

1. We model the temporal dependency of historical trip observations to forecast the number of incoming and outgoing trip requests for each city region (node). We achieve this by using Gated Recurrent Units (GRUs). 
2. We preform trip demand predictions using a convolutional neural network (CNN). However, in contrast to other methods in the literature that rely on raster images or adjacency matrices, our method captures the correlations between the features of origin and destination regions (i.e., the nodes of an edge) through a novel approach that exploits contextual data to learn latent relationships.

\subsection{Contribution Summary}

Our contributions in this work are four fold:

- To our knowledge we are the first to introduce the use of demographic and socio-economic data with land-use categories as features of city regions to train a machine learning model to perform city-wide trip demand forecasting.

- A novel framework that first predicts incoming and outgoing trip requests per city region, and then exploits these predictions to enhance the forecasting accuracy of trip demand between origin and destination region pairs.

- Introduce a method to assemble a matrix based on the feature representations of origin and destination regions to extract spatial correlations from the data using convolutional operations with specific filter dimensions on non-image data and effectively generate trip demand.

- Evaluate our proposed framework on the New York City taxi dataset. The results show that the CityNet model can outperform several state-of-the-art methods, including two variations of the proposed framework. 


\subsection{Thesis Organization}

We organize this thesis as follows: Chapter 2 presents some of the forecasting methods used in the literature and summarizes the relevant research. In Chapter 3 we define the research problem and detail our proposed CityNet model, including the learning pipeline used to train it. In Chapter 4 we present the socio-economic and land-use features used as input, the trip record dataset employed for training and testing, and we discuss the results of our experiments and findings. Lastly, in Chapter 5 we give a summary of the research work presented here, point out open problems, and suggest future research. 


\section{Chapter 2}

\section{Literature Review}

A large and diverse body of research, reviewed in the following surveys and references within $[8,17,9,7]$, proposed to solve traffic prediction problems using various forecasting techniques and complex hybrid models in a data-driven approach. According to [7], these methods can be organized into two broad categories. 1) traditional methods that involve classical statistical and machine learning models, and 2) deep learning-based methods built on neural network architectures. In this chapter, we discuss the research that focused on traffic demand and flow forecasting, which are the two traffic problems addressed by our work. We organize the chapter as follows. In Section 2.1 we discuss the literature that applies traditional forecasting methods for traffic prediction, followed by the literature that utilizes deep learning-based methods in Section 2.2. 


\subsection{Traditional Methods}

Classical statistical models and machine learning methods were the main forecasting techniques used by researchers in traffic forecasting literature until the advent of deep learning-based methods [7]. In Table 2.1 we show a summery of the discussed techniques in this section.

Table 2.1: Summery of the traffic forecasting techniques discussed in Section 2.1

\begin{tabular}{|c|c|c|c|}
\hline Method Name & Author & Year & Short Description \\
\hline ARIMA & $\begin{array}{l}\text { S. Shekhar and B. } \\
\text { Williams [18] }\end{array}$ & 2008 & $\begin{array}{l}\text { Proposed adaptive parameter adjustment using filtering } \\
\text { techniques }\end{array}$ \\
\hline ARIMA-GARCH & Chen et al. [19] & 2011 & Captures the volatility of traffic data using GARCH \\
\hline ARIMA & Abadi et al. [20] & 2015 & $\begin{array}{l}\text { Addressed limited traffic data using a link-to-link propaga- } \\
\text { tion method }\end{array}$ \\
\hline Bayesian network & Zhang et al. [21] & 2004 & $\begin{array}{l}\text { Conceptualized the methodology of using a Bayesian net- } \\
\text { work for traffic forecasting }\end{array}$ \\
\hline $\begin{array}{l}\text { Spatio-temporal } \\
\text { Bayesian network }\end{array}$ & Sun et al. [22] & 2005 & Applies a selective variable process to reduce computation \\
\hline $\begin{array}{l}\text { Gaussian } \\
\text { Bayesian } \\
\text { work }\end{array}$ & $\begin{array}{l}\text { Castillo et al. } \\
{[23]}\end{array}$ & 2008 & $\begin{array}{l}\text { Uses the Origin-Destination sets as parents in the Bayesian } \\
\text { network and the traffic flow as their nodes }\end{array}$ \\
\hline SVM & Zhang et al. [24] & 2011 & Combines seasonal ARIMA and SVM \\
\hline SVR & Lippi et al. [25] & 2013 & Combines SVR with a seasonal kernel \\
\hline
\end{tabular}

\subsubsection{Classical statistical models}

Classical approaches were lead by the Box-Jenkins family of models [25], which apply an Autoregressive Integrated Moving Average (ARIMA) for prediction. The Autoregressive part indicates that the target variable is regressed on its lagged values, while the Moving Average indicates that the forecast error is a linear combination of past errors. The Integrated component is a differencing factor between the target and past observations, making the data stationary. The ARIMA model and it's variants are one of the most consolidated approaches to solving trip demand prediction problem 
as a time-series forecasting task [7].

Many past studies $([6,20,19,26,18,27,28])$ have shown the applicability of the ARIMA models with various traffic data complexities. S. Shekhar and B. Williams [18] proposed an adaptive approach for model parameters adjustment without the need for re-estimation through the use of filtering techniques (e.g., Kalman filter, least mean squares), as well as account for the possibility of missing data records during the model fitting process. Other researchers, such as Abadi et al. [20], address the issue of limited traffic data by using the ARIMA model with a link-to-link dividing ratio methodology as an approach to propagate predicted traffic from specific links to adjacent links.

Moreover, Chen et al. [19] discuss how an ARIMA model combined with a Generalized Autoregressive Conditional Heteroscedasticity (ARIMA-GARCH) process, which models the current variance in the data based on past observations and past variances, can capture the volatility of traffic data. However, the authors state that due to the complexity of the model a standard ARIMA is sufficient for individual traffic sensor (loop detector) forecasting. Further, Lippi et al. [25] show, through experimental results on several traffic stations, that seasonal ARIMA with a Kalman filter achieves superior results to that of support vector regression models. The authors highlight that seasonality is a key feature of trip data.

Nevertheless, ARIMA and it's variants are designed for small datasets, and are univariate methods with high computational cost for multivariate approaches. They are a single-point estimators incapable of modelling city-wide trip data, and impose strong stationary on the data, which is not the case for many dynamic, high traffic, 
cities. These short-comings make ARIMA and it's variants unsuitable for practical real-time situations.

\subsubsection{Machine Learning Methods}

Machine learning algorithms were used by researchers to handle the complexity and time dependence of trip data. These algorithms can accommodate multivariate data and capture complex non-linear relationships. However, the performance of these methods are still limited because they require hand-crafted features, rather than learning directly from the raw data.

\subsubsection{Bayesian Networks}

Zhang et al. [21] conceptualized the methodology of using a Bayesian network to model and forecast traffic flow. A Bayesian network is a probabilistic graphical model coined by J. Pearl [29]. It employs a directed acyclic graph to predict the likelihood of a single or several factors in producing an event. The proposed method in [21] relies on the Bayesian network's ability to take into account the influence of neighbouring nodes on the current node, where nodes of the graph represent road links (traffic flow) in a transportation network. The authors mention that the traffic on a link is related to the traffic of neighbours and itself in past time intervals, and they consider the time factor as Bayesian network models. This approach uses the Gaussian Mixture Model (GMM), which is a probabilistic model that assumes all points in a dataset are generated from a mixture of Gaussian distributions, to approximate the joint probability distribution of all nodes on one constructed Bayesian network. The results 
reported in the paper show improved error rates over autoregressive models.

Moreover, Sun et al. [22] proposed a more efficient approach for traffic flow prediction. They utilize a Bayesian network model that incorporates spatial and temporal information in the transportation network with a variable ranking procedure. The authors mention that the selective variable process eliminates redundant variables (traffic flows), which reduces computational cost. The method employs the Pearson Correlation Coefficient [30], a measure of linear correlation between two datasets, as the specific ranking criterion, followed by the best-first search strategy to select the most relevant variables from the ranking result as final nodes of a Bayesian network. The experimental results show that the proposed method can outperform Random Walk and Markov Chain models with varying error rates over different road segments.

Furthermore, an alternative approach proposed by Castillo et al. [23] estimates traffic flow between two adjacent points in the transportation network, as well as the Origin-Destination (OD) set using a Gaussian Bayesian Network. The method uses the OD sets as parents in the Bayesian network and the traffic flow as their nodes, and rely on the networks ability to provide the joint density of all unobserved variables (traffic flows) for predictions. However, the Bayesian network suffers from similar drawbacks as the ARIMA model, and require large computational costs for considering several past time intervals. It also depends heavily on the network design, which is a crucial limitation. 


\subsubsection{Support Vector Machines}

Support Vector Machines (SVM) [31] are supervised machine learning methods that aim to find a hyperplane that maximizes the margin between each class data points in an N-dimensional space. New data is then classified by falling on either side of the hyperplane. Support Vector Regression (SVR) [32] builds on SVM by minimizing the coefficients subject to a constrain function.

Zhang et al. [24] shows that combining a seasonal ARIMA model with a support vector machine (SVM) provides superior results than the individual models. The authors mention that the hybrid model can address the periodicity, nonlinearity, and complexity of short-term traffic forecasting by leveraging the strengths of the individual models. The input variables to the SVM are determined by means of the SARIMA modeling principle, and the SVM parameters are further optimized using particle swarm optimization.

Moreover, Lippi et al. [25] proposed two Support Vector Regression (SVR) models that benefit from the seasonality of trip demand time-series data. The approach utilizes a 1) Radial Basis Function (RBF) kernel or a 2) linear kernel function, with a seasonal kernel, which represents the temporal distance between two records. By exploiting the trip records timestamps, the seasonal kernel facilitates the detection of similar behaviors with past time intervals in the transportation network. The experimental results in the paper show that the seasonal ARIMA model provides lower error rates than the proposed SVR models. However, both seasonal SVR models show a good compromise between accuracy and computational time when compared to the high computational time of the seasonal ARIMA model. 


\subsection{Deep Learning-based Methods}

Vlahogianni et al. [9] and Yin et al. [7] have conducted intensive reviews of the existing literature on short-term traffic prediction, and observed that most researchers are diverging from traditional statistical methods to Deep Learning-based (DL) approaches. DL methods have shown great success in understanding the complex nonlinearity and capturing the spatio-temporal correlation of time-series traffic data, including the exogenous factors that affect them. DL models are nested hierarchical models that incorporate various architectures of neural networks (NN). In this section we detail the NN structures used for traffic flow forecasting according to how they model the spatio-temporal correlation, and further discuss the relevant literature. In Table 2.2 we show a summery of the discussed techniques in this section.

\subsubsection{Fully Connected Neural Network}

A Fully Connected Neural Network (FCN) is a network such that every neuron in a layer is connected to every neuron in the next layer. A classical example of this type of network is a Multilayered Perceptron (MLP). Various FCN architectures have been proposed to solve the traffic forecasting problem. N. Polson and V. Sokolov [34] proposed a DL architecture that captures the nonlinear spatio-temporal effects of traffic data through the use of a linear layer that is fitted using lasso regression, combined with a sequence of hyperbolic tangent, tanh, activated layers. Their results show that deep (i.e., multilayered) model can achieve significant improvements over a shallow linear model. Moreover, Lv et al. [33] proposed a novel DL approach to discover the latent traffic feature representations, by stacking autoencoder layers 
Table 2.2: Summery of the traffic forecasting techniques discussed in Section 2.2

\begin{tabular}{|c|c|c|c|}
\hline Method Name & Author & Year & Short Description \\
\hline FCN & Lv et al. [33] & 2015 & Stacked autoencoders \\
\hline FCN & $\begin{array}{l}\text { N. Polson and V. } \\
\text { Sokolov [34] }\end{array}$ & 2017 & $\begin{array}{l}\text { Showed that multilayered models are superior to linear } \\
\text { models }\end{array}$ \\
\hline DeepST & Zhang et al. [11] & 2016 & $\begin{array}{l}\text { Applied CNNs to capture the spatial dependency in the } \\
\text { data }\end{array}$ \\
\hline ST-ResNet & Zhang et al. [1] & 2017 & $\begin{array}{l}\text { Improves on DeepST by incorporating residual layers in the } \\
\text { CNN architecture }\end{array}$ \\
\hline ST-3DNet & Guo et al. [10] & 2019 & Uses 3D convolution for time-series traffic forecasting \\
\hline $\mathrm{RNN}$ & $\begin{array}{l}\text { D. Zhang and M. } \\
\text { Kabuka }\end{array}$ & 2017 & Utilizing weather information for traffic prediction \\
\hline RPA-LSTM & Niu et al. [35] & 2019 & A dynamic city partitioning and feature merging method \\
\hline STRCN & Jin et al. [36] & 2018 & $\begin{array}{l}\text { Removes the residual units from the ST-ResNet model, and } \\
\text { adds LSTM layers after the CNN }\end{array}$ \\
\hline DMVST-Net & Yao et al. [3] & 2018 & $\begin{array}{l}\text { Combines a CNN, LSTM, and a feature embedding layer } \\
\text { into a framework }\end{array}$ \\
\hline $\begin{array}{l}\text { DCRNN } \\
\text { GRU })\end{array}$ & Li et al. $[14]$ & 2018 & $\begin{array}{l}\text { Replaces the matrix multiplication operation in RNNs with } \\
\text { a diffusion convolution operation }\end{array}$ \\
\hline ST-GCN & Yu et al. [4] & 2018 & $\begin{array}{l}\text { Relies entirely on convolutional structures to capture the } \\
\text { spatio-temporal correlations in the data }\end{array}$ \\
\hline TGC-LSTM & Cui et al. [37] & 2020 & $\begin{array}{l}\text { Incorporates a Traffic Graph Convolution operation as part } \\
\text { of the LSTM cell }\end{array}$ \\
\hline ST-MGCN & Geng et al. [13] & 2019 & $\begin{array}{l}\text { Uses contextual gating mechanism to produce region de- } \\
\text { scriptions }\end{array}$ \\
\hline DGCNN & Diao et al. [16] & 2019 & $\begin{array}{l}\text { Introduced a Laplacian matrix estimator to estimate the } \\
\text { adjacency matrix of the graph }\end{array}$ \\
\hline DST-GCNN & Chen et al. [15] & 2020 & $\begin{array}{l}\text { Utilizes an adjacency matrix derived from travel time be- } \\
\text { tween locations }\end{array}$ \\
\hline STDN & Yao et al. [38] & 2018 & $\begin{array}{l}\text { Employs a flow gating mechanism to capture the dynamic } \\
\text { spatial similarities in traffic data }\end{array}$ \\
\hline ST-MetaNet & Pan et al. [5] & 2019 & $\begin{array}{l}\text { Uses an encoder-decoder framework with an RNN, meta } \\
\text { learning, and GAT layers. }\end{array}$ \\
\hline GSTNet & Fang et al. [12] & 2019 & $\begin{array}{l}\text { Applies dilated causal CNN to extract short-term neigh- } \\
\text { boring and long-term periodic dependencies from historical } \\
\text { data }\end{array}$ \\
\hline
\end{tabular}

followed by a predictor layer. Their results show that the proposed DL model can achieve lower error rates than that of SVM, Random Walk, and the Radial Basis Function NN models. 


\subsubsection{Convolution Neural Network}

The Convolution Neural Network (CNN), developed by LeCun et al. [39], has shown significant strides in image classification $([40,41,42])$ and object detection $([43,44$, 45]), due to its ability to extract spatial patterns such as relations between image pixels. In traffic flow forecasting literature, several studies $([1,3,10,11,46])$ found great success in converting a city's traffic data into a two-dimensional grid (raster data, illustrated in Figure 2.1), where each cell represents a region of the transportation network with traffic information. Each constructed 2D grid represents a single time step in the time-series data. This approach facilitates the use of CNNs to capture the spatial dependence of the traffic data on the transportation network. As an example, by using a $3 \times 3$ kernel every region is surrounded by eight neighbours to draw information from, for each convolution operation. With every subsequent convolution layer in the network, the model can capture spatial dependency of near, and with enough layers, far interregional correlations.

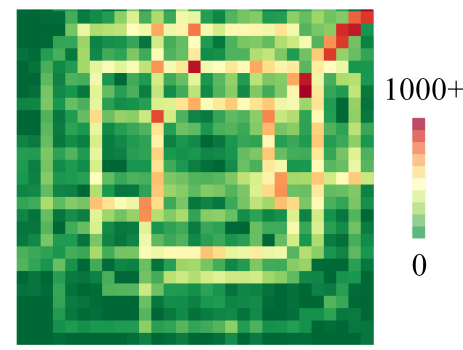

Figure 2.1: Example of traffic data as a 2D grid (raster image) used for convolution (partial image taken from [1]).

Moreover, convolution operations have also been applied for temporal dependency modeling of traffic data in the form of causal convolution $([16,4])$ and dilated causal 
convolution ([12]). Causal convolution is a normal one-dimensional convolution applied on time axis, illustrated in Figure 2.2a. On the other hand, dilated causal convolution, developed by van den Oord et al. [2] on audio signals for text-to-speech modeling, is a form of one-dimensional convolution where the kernel is applied over an area larger than its length by skipping input values with a certain step (dilation rate). Figure $2.2 \mathrm{~b}$ illustrates the technique. The dilation allows the model to capture long-term temporal dependencies more effectively with every stacked layer.

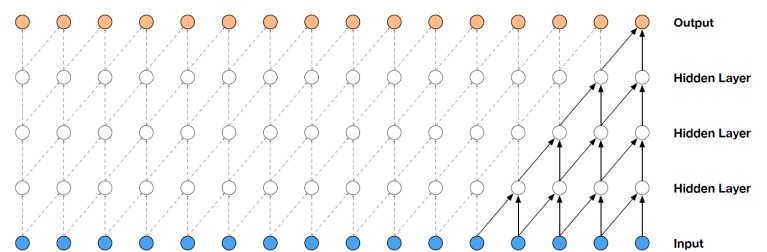

(a)

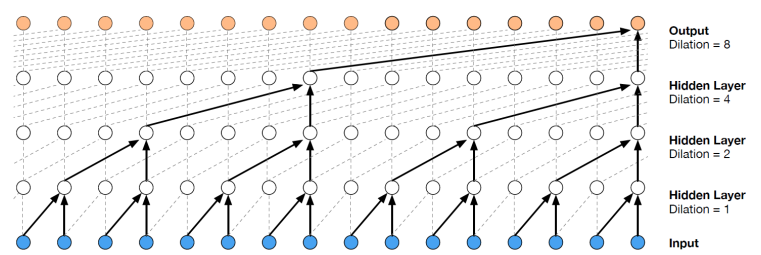

(b)

Figure 2.2: Illustration of a stack of (a) causal convolution, and (b) dilated causal convolution layers (image taken from [2]).

\subsubsection{Research work that applies CNN for traffic forecasting}

Zhang et al. [11] in 2016 proposed a deep spatio-temporal (DeepST) model for traffic forecasting. Their architecture first distinguishes the temporal dependent instance as closeness, period and seasonal trend. Then they apply CNNs to capture the spatial dependency of each instance. Lastly, they apply fusion operations on the latent representations to perform traffic predictions. In 2017, Zhang et al. [1] proposed a spatio-temporal residual network (ST-ResNet) that improves on their previous architecture by employing residual neural networks to better model the temporal closeness, 
period and seasonal trends. Figure 2.3 illustrates the ST-ResNet architecture. The results of the research show that ST-ResNet can outperform ARIMA, SARIMA, VAR, and DeepST models by a significant margin, more so by increasing the number of residual units in the framework.

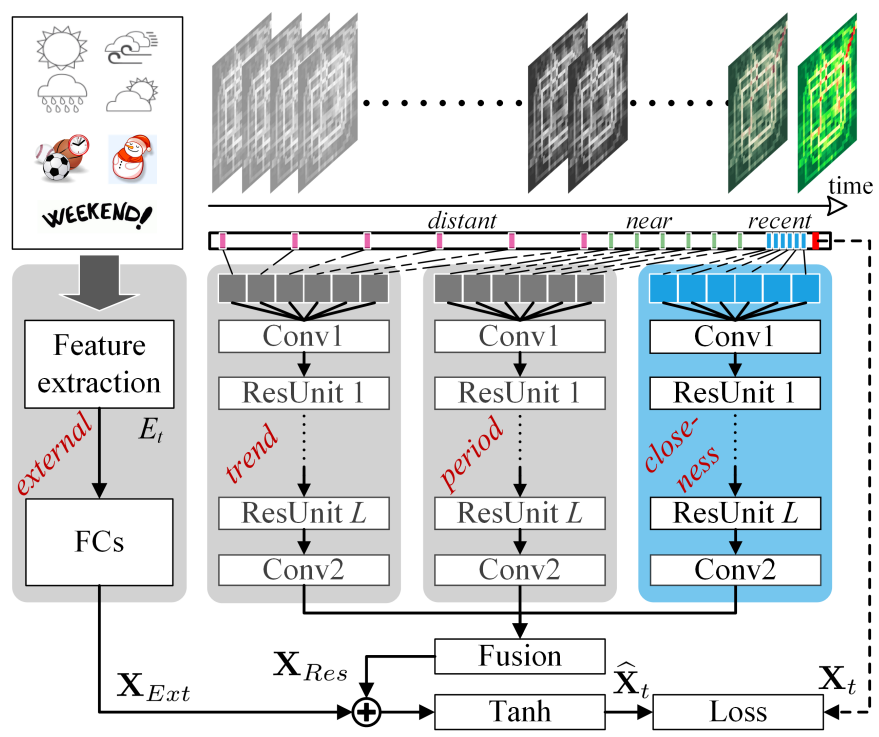

Figure 2.3: ST-ResNet architecture, where Conv is Convolution, ResUnit is Residual Unit, and FC is Fully-connected (image taken from [1]).

Further, inspired by the success of 3D CNN on video analysis, Guo et al. [10] proposed the ST-3DNet model that utilizes 3D convolution for time-series traffic forecasting. At each time step, the traffic information can be interpreted as a video frame. The convolution operation applies a 3D kernel (filter) on several frames of traffic data, which yields a 3D feature map that preserves temporal information. This process effectively captures the temporal dependency of traffic motion with every subsequent convolution layer. Similar to ST-ResNet, the ST-3DNet models the temporal closeness and weekly period of traffic data and applies fusion operations to merge the latent representations to perform predictions. The paper shows that ST-3DNet 
can achieve improved results over ST-ResNet, more so when considering exogenous factors.

Nonetheless, a significant drawback of the spatio-temporal CNN models is that they imposes strong spatial constraints on the data, which is not ideal for an expanding and changing city transportation network.

\subsubsection{Recurrent Neural Network}

Recurrent Neural Networks (RNN) are a form of neural networks where the connection between the neurons form a directed graph along the temporal axis. RNNs utilize a hidden state to carry past information across the network, and allow previous outputs to be used as inputs. This structure makes RNNs highly efficient in learning sequential data, including handwriting recognition and generation $([47,48])$, natural language processing $([49,50,51,52])$, audio and video analysis $([53,54])$, and protein structure prediction ([55]) among others. They have also been utilized to model the temporal dependency that is present in time-series traffic data $([36,56,35,3,57])$. Two renowned RNN variants that have shown great success in recent years are Long Short-Term Memory (LSTM) and Gated Recurrent Unit (GRU).

LSTM and GRU are gated mechanisms of the RNN architecture developed by S. Hochreiter \& J. Schmidhuber [58] and Cho et al. [52], respectively. They learn correlations within the data by controlling the flow of information inside the unit with gating functions. 


\subsubsection{Research work that applies RNN for traffic forecasting}

D. Zhang and M. Kabuka [57] proposed a vanilla RNN architecture with GRU layers to perform traffic flow predictions. In their research, the authors indicate the importance of weather conditions in influencing traffic flow. In addition to traffic data, for each time step, the input feature vector includes weather type, precipitation, average wind speed, and average temperature. The results show that predicting traffic flow by exploiting weather information produces improved prediction accuracy over no weather information.

Niu et al. [35], proposed a Region Partition Assisted Long Short-Term Memory (RPA-LSTM) model. The novelty in the model is a dynamic partitioning method that first divides a target city into blocks by considering the characteristics of each block. The method then combines blocks with similar characteristics. The final partitioning map is then processed by an LSTM network. Their approach highlights the importance of human mobility and travel patterns between distinct regions of a city. In the paper, the authors report improved error rates of their proposed partitioning technique over Equal Area and Road Network-based partition methods, even when used in conjunction with an ARIMA model.

Moreover, in several traffic flow forecasting studies researchers have combined various neural network architectures into a single complex structure to exploit each model's strengths and mitigate its shortcomings. For example, Jin et al. [36] introduced an alteration to the ST-ResNet model for crowed flow prediction, named Spatio-Temporal Recurrent Convolutional Networks (ST-RCN). The proposed architecture removes the residual units from the ST-ResNet model, and adds LSTM layers 
after the CNN. The authors note that the residual units are not necessary to capture the spatial dependencies of regions that are far away from the targeted region. The reported results show a significant improvement of ST-RCN over ARIMA, LSTM, and ST-ResNet.

Further, the Deep Multi-View Spatial-Temporal Network (DMVST-Net), proposed by Yao et al. [3] for taxi demand prediction, combines a CNN, LSTM, and a feature embedding layer into a framework with three conceptual views (Figure 2.4): 1) The spatial view utilizes a CNN to extract spatial correlations within traffic raster

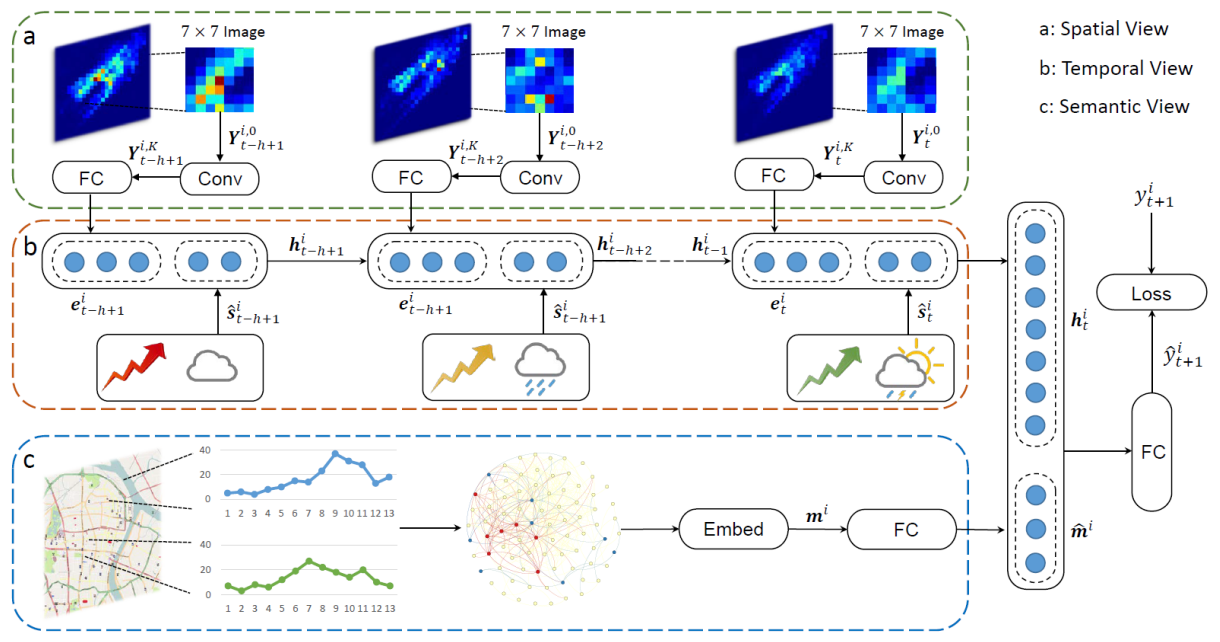

Figure 2.4: DMVST-Net architecture, where Conv is Convolution, FC is FullyConnected, Loss is the loss function, and Embed is feature embedding (image taken from [3]).

data for each time step. 2) The temporal view sequentially processes the latent representations derived from the spatial view, in conjunction with weather information, using an LSTM network. 3) The semantic view leverages an embedding layer to extract similarities between the regions of a city represented as a graph. The city-wide prediction is then acquired by applying a non-linear function on the concatenation of 
the temporal view and the semantic view feature representations. In the paper the authors report a significant margin of improvement in prediction error rates using their framework over ARIMA, least square regression, ridge regression, ST-ResNet among others methods.

\subsubsection{Graph Convolution Network}

The Graph Convolution Network (GCN) is a neural network that applies convolution operations on graph structured data by algebraically processing its adjacency matrix, degree matrix, and feature matrix to understand the impact of neighboring nodes on the selected node. The GCN is capable of modeling the spatial dependency of non-Euclidean data structures, and has been successfully utilized to model the transportation network for traffic forecasting $([4,14,15,16,37])$. There are three forms of GCN:

- Spectral method, developed by Bruna et al. [59], applies convolution on graph data from the spectral domain by using graph Fourier transform on a Laplacian matrix. Due to the computational complexity of this method two approximation strategies have been proposed, ChebNet [60], which introduced a filter as Chebyshev polynomials of the diagonal matrix of eigenvalues, and first order of ChebNet (1stChebNet) [61], which is a first-order approximation of ChebNet.

- Spatial method, developed by Niepert et al. [62], constructs locally connected neighborhoods from the input graph. These neighborhoods are mapped from a graph representation into a vector space representation and serve as the receptive fields of a convolutional layer, allowing the model to learn. 
- Diffusion method, developed by Atwood, J. and Towsley D. [63], applies a mapping from nodes to the output of a diffusion process. The parameters of a diffusion convolution operation are tied according to a diffusion search depth rather than their position in a grid. The authors mention that ChebNet can be considered as a special case of diffusion convolution.

\subsubsection{Research work that applies GCN for traffic forecasting}

Li et al., in their paper [14] proposed a novel Diffusion Convolution Recurrent Neural Network (DCRNN), which replaces the matrix multiplication operation in RNNs with a diffusion convolution operation. The study utilizes an encoder-decoder model for traffic forecasting, with several diffusion convolution recurrent layers. The experimental results show that the proposed architecture can outperform ARIMA, vector auto-regression, SVR, a feed-forward NN, an LSTM model, and, most prominently, ChebNet.

Yu et al. [4], proposed a Spatio-Temporal Graph Convolutional Network (STGCN) that relies entirely on convolutional structures to capture the spatio-temporal correlations. The ST-GCN architecture, illustrated in Figure 2.5, consists of two ST-convolution (ST-Conv) blocks followed by an output layer. Each block contains two temporal gated convolution layers separated by a spectral graph convolution layer (ChebNet) in the middle. The gated convolution layer is structured as a causal convolution operation, a form of 1D convolution applied on temporal data, connected to a gated linear unit with residual connections. The significance conveyed in this research is that the ST-GCN model can achieve lower error rates than GCGRU, which is a GRU variant of the DCRNN model mentioned in section 2.2.4, and it can 
be trained 14 times faster due to the temporal gated convolution layer.

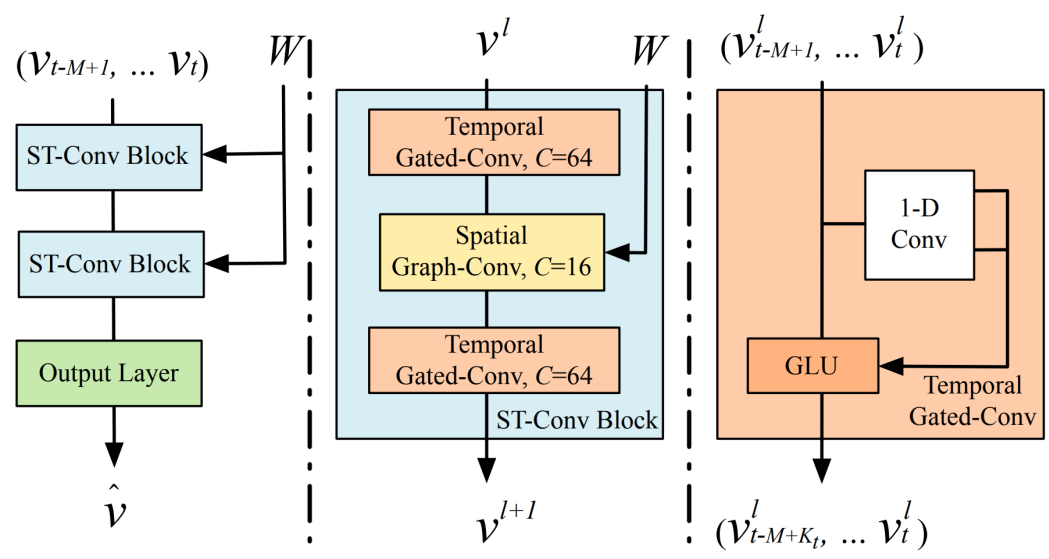

Figure 2.5: An illustration of the ST-GCN architecture, where $V^{t}$ is a finite set of vertices corresponding to traffic observations and $W$ denotes the weighted adjacency matrix of the graph (image taken from [4]).

Moreover, Cui et al. [37] proposed a Traffic Graph Convolutional LSTM (TGCLSTM) architecture, where the TGC operation is incorporated as part of the LSTM cell. TGC, which is a form of spatial GCN, introduces a novel method to compute the convolution output feature vector. It is computed by applying the element-wise product on a weighted adjacency matrix, $k$ - hop neighbourhood adjacency matrix, where $k$ adjacent nodes are considered, and a "free-flow reachable" matrix, which represents the possibility of reaching a node within a fixed number of time steps by 1 , and 0 otherwise. The experimental results compare the TGC-LSTM methods most notably with spectral GCN+LSTM, localized spectral GCN+LSTM, and an adjusted version of DCRNN where the diffusion convolution is replaced with spectral graph convolution. The results show that TGC-LSTM can significantly outperform localized spectral GCN+LSTM and the adjusted version of DCRNN. However, it marginally improves the error rates over the spectral GCN+LSTM method. 
Geng et al. [13], proposed a Spatio-Temporal Multi-Graph Convolution Network (ST-MGCN) for ride-hailing demand forecasting. Their approach divides a target city into a grid, and then extracts three spatial components as graphs: neighbourhood, functional similarity, and transportation connectivity. To model the temporal correlations in the graphs a contextual gated $R N N$ is proposed. The contextual gating mechanism produces region descriptions by concatenating the historical data of a region with data from neighbouring regions, which is extracted by a spectral graph convolution operation. After the contextual gating, a single RNN layer is applied across all regions to aggregate the descriptions. Then, a MGCN is used to model and aggregate different spatial correlations among the regions of the graph. Lastly, an FCN is used to generate the demand predictions from the aggregated feature vectors. The results of the study show that the proposed ST-MGCN model benefits greatly from the extracted components each slightly improving the error rates. In addition, the ST-MGCN can outperform ST-ResNet, DMVST-Net, and ST-GCN by a considerable margin.

Furthermore, most GCN models rely on a static form of the adjacency matrix to carry the convolution operation. Diao et al. [16] presented a dynamic form of the spectral GCN, named Dynamic Spatio-Temporal Graph Convolution Neural Network (DGCNN). The novelty is the introduction of a Laplacian matrix estimator. The estimator contains two components: a tensor decomposition layer (TDL) and a dynamic Laplacian matrix learning unit. The TDL extracts global and local component of the traffic data, and is pre-trained to initialize its parameters before training the entire framework. The extracted global and local components are used by the Laplacian matrix learning unit to perform Laplacian matrix estimations. The reported results 
in the study indicate that DGCNN can produce marginally lower error rates than GCGRU, and ST-GCN.

In addition, a dynamic form of the spatial GCN was proposed by Chen et al. [15]. Their model, named Dynamic Spatio-Temporal Graph-based CNN (DST-GCNN), utilizes an adjacency matrix derived from travel time between locations. The DSTGCNN consists of two prediction steps: 1) graph prediction, which predicts a future adjacency matrix based on historical adjacency matrices using several convolution layers, and 2) flow prediction, which uses the predicted adjacency matrix with spatial GCN to predict future traffic flow for the entire graph. This two-step process captures the dynamic changes in graph structured data. The experimental results indicate that DST-GCNN can slightly outperform DCRNN and ST-GCN.

\subsubsection{Attention Mechanism}

The traffic flow on a road segment in a transportation network is impacted by neighboring (or intersecting) roads in various degrees. These impacts are dynamic and vary over time. To model these properties and adaptively capture the interregional correlation in the transportation network, researchers have used attention mechanisms, as well as graph attention networks (GAT) $([12,64,5,38])$. GAT was developed by Veličković et al. [65]. The attention method applies a linear transformation as a weighted sum of the input feature vectors, which results in an attention map. The weight coefficients (attention coefficients) determine the importance of the neighboring features. Additionally, multi-head attention can also be computed to improve the learning process by aggregating multiple attention maps for each input vector. 


\subsubsection{Research work that applies GAT for traffic forecasting}

Yao et al. [38] introduced a novel Spatio-Temporal Dynamic Network (STDN) for traffic flow prediction. The model employs a flow gating mechanism that utilizes CNNs to capture the dynamic spatial similarities in traffic flow and traffic volume between regions. Then, for each time step, an LSTM is applied to capture the temporal dependency of the traffic representation, followed by a periodically shifted attention mechanism that captures the long-term periodicity from the LSTM feature vectors (i.e, the relevant features). The experimental results show that STDN can achieve lower error rates than DeepSD, ST-ResNet, and DMVST-Net.

Moreover, Pan et al. [5] applied an encoder-decoder framework with a network structure based on RNN, meta learning, and GAT. The architecture, entitled STMetaNet and illustrated in Figure 2.6, is the same for encoder (blue parts) and decoder (green parts). It includes four components: 1) An RNN that is used to

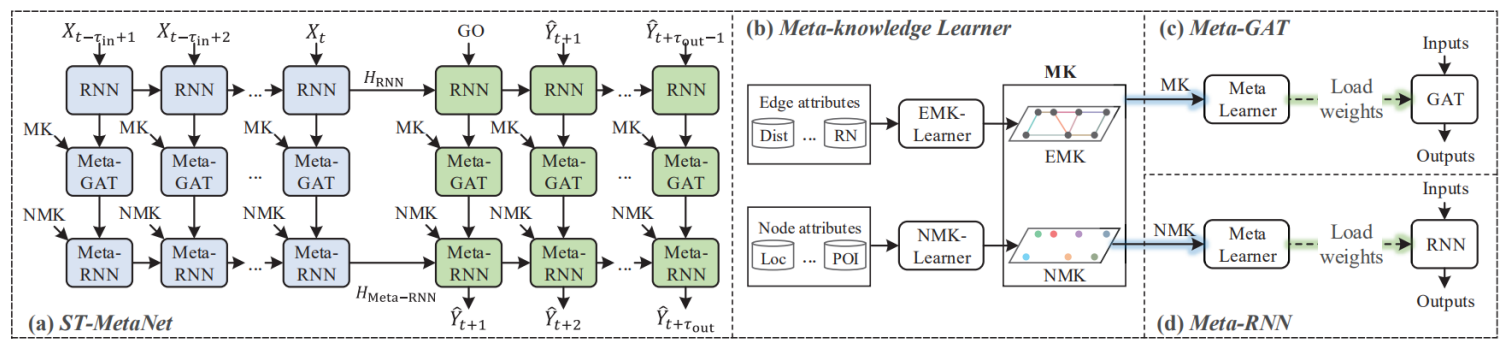

Figure 2.6: An illustration of the ST-MetaNet architecture, where $X_{t}$ is a vector corresponding to traffic observations and $\hat{Y}_{t}$ an output vector corresponding to traffic predictions at time $t$, and $H_{R N N}$ and $H_{M e t a-R N N}$ denote the hidden states (image taken from [5]).

learn the temporal correlations of the historical traffic data. 2) Two fully connected NNs (FCN) called node meta knowledge learner (NMK) and edge meta knowledge learner (EMK), to respectively learn the node attributes and edge attributes. 3) A 
Meta-GAT, which employs a FCN to learn and initialize the weights of GAT based NMK and EMK. The Meta-GAT learns the spatial correlations of the graph. For each node, Meta-GAT mainly performs two steps: attention score calculating over each edge, and hidden state aggregation (Figure 2.7, shows an example operation of Meta-GAT). 4) A Meta-RNN, which employs a FCN to learn and initialize the weights of RNN for each location based on NMK. The Meta-RNN captures the dynamic temporal correlation of the associated locations. In the paper, the authors report that

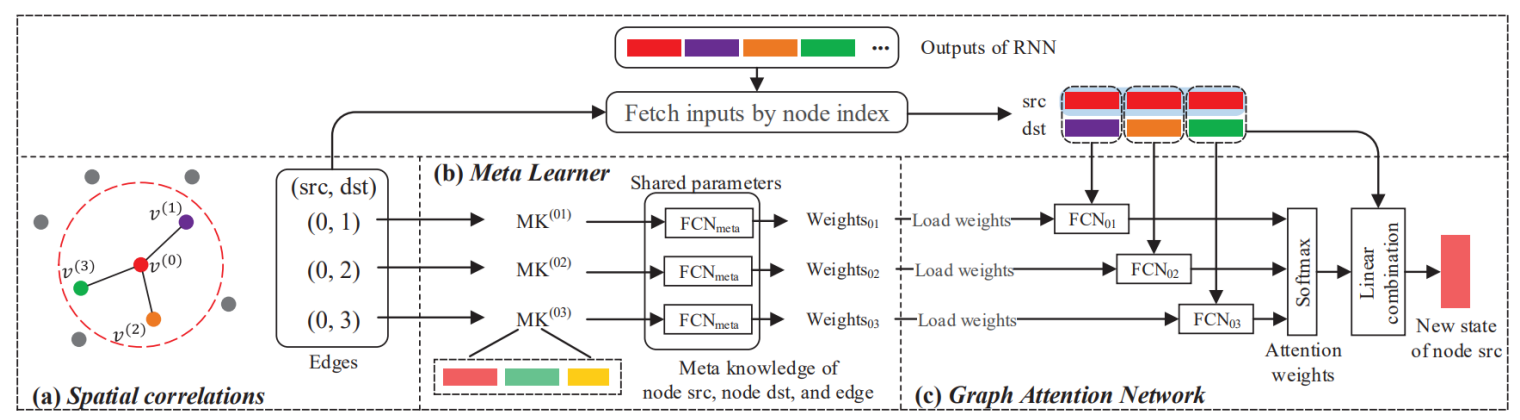

Figure 2.7: An example of Meta-GAT that calculates the impact to the red node from its neighborhoods along edges (image taken from [5]).

the experimental results of ST-MetaNet outperform a generic GAT-seq2seq model, ST-ResNet and DCRNN. Most notably ST-MetaNet requires 34\% less training parameters in its network than ST-ResNet and DCRNN.

Moreover, Fang et al. [12], introduced a Global Spatio-Temporal Network (GSTNet) for traffic flow prediction. The model utilizes a dilated causal CNN to extract short-term neighboring and long-term periodic dependencies from historical data for all nodes. The output vectors, for all nodes in the transportation network, are then processed by a localized GCN to find local spatial dependencies. Subsequently, a global spatial correlation mechanism is applied to capture non-localized spatial correlations between the nodes. The resulting output features, for all nodes, are further 
processed by an attention mechanism on the temporal axis by using a Frobenius inner product of two matrices. The function measures the relevance of the output features between the historical and current time steps, which are finally mapped into traffic states on the network. The experimental results of the study indicate that GSTNet can outperform ChebNet, GCGRU, ST-GCN by a significant margin. Notably, the study also indicates that by removing either the temporal or spatial modules the performance of the model suffers significantly. 


\section{Chapter 3}

\section{Methodology \& Implementation}

In this chapter, we present the methodological framework and implementation steps needed to construct our proposed CityNet Model. In Section 3.1 we introduce our preliminary definitions and notations used throughout the thesis, followed by a formal definition of the trip demand prediction problem in Section 3.2. Finally, we present our solution to the trip demand problem in Section 3.3, where we detail the different parts of our developed model, ensued by the learning pipeline employed to train it.

\subsection{Preliminaries}

We begin by presenting the graph notation. We denote a fully connected directed graph structure as $G=(V, E)$, where $V(|V|=n)$ is the set of nodes $\left\{v_{1}, \ldots, v_{n}\right\}$, and $E\left(|E|=m=n^{2}\right)$ is the set of edges $\left\{e_{1}, \ldots, e_{m}\right\}$. Each edge $e \in E$ is an ordered pair of nodes denoted as $e=(v, u)$, where $v, u \in V$ and is directed from $v$ to $u$. For 
a node $v$, we denote the set of incoming edges to the node as $I N(v)$, and the set of outgoing edges from the node as $O U T(v)$.

In the following section we define our city representation, which we impose on the graph structure.

\subsubsection{City Representation}

The set of nodes, $V$, represents non-overlapping regions of a city that are connected via directed edges, and the set of edges, $E$, represents the flow of traffic between the regions. The graph is fully-connected since there can be trips between every pair of regions. In addition, the graph also includes self-loops, i.e., $e=(v, v)$. We associate the nodes and edges of the graph with specific attributes, as well as global attributes that affect both at each time interval, as shown in Figure 3.1.1.

Node Attributes We associate each node with the attributes of the region: demographic, social, and economic features. Additionally, we use land-use $e^{1}$ data to categorize the nodes with specific types. The categories help capture different relations across the nodes, such as traffic flowing from housing to business districts. We denote the node attributes as a vector $A_{v} \in \mathbb{R}^{\alpha^{V}}$, where $\alpha^{V}$ is the number of attributes.

Edge Attributes Edges are associated with the route distance (on a road network) between their adjacent nodes, as well as the time varying trip demand values that

\footnotetext{
${ }^{1}$ Land-Use information pertains to the zoning regulations and designation of properties in a city, such as building dimensions and land classification.
} 
are precomputed from raw ride-hailing trip records (detailed in the Section 3.2). We denote the edge attributes as a vector $A_{e} \in \mathbb{R}^{\alpha^{E}}$, where $\alpha^{E}$ is the number of attributes.

Global Attributes The global attributes are time-dependent high-level features that influence both the nodes and edges. The set of attributes contains meteorological information, as well as information related to the current date and time, such as day of the week. These features facilitate more intricate patterns to occur. For a given time interval $t$, we denote the global attributes as a vector $\mu^{t} \in \mathbb{R}^{\alpha^{\mu}}$, where $\alpha^{\mu}$ is the number of attributes.

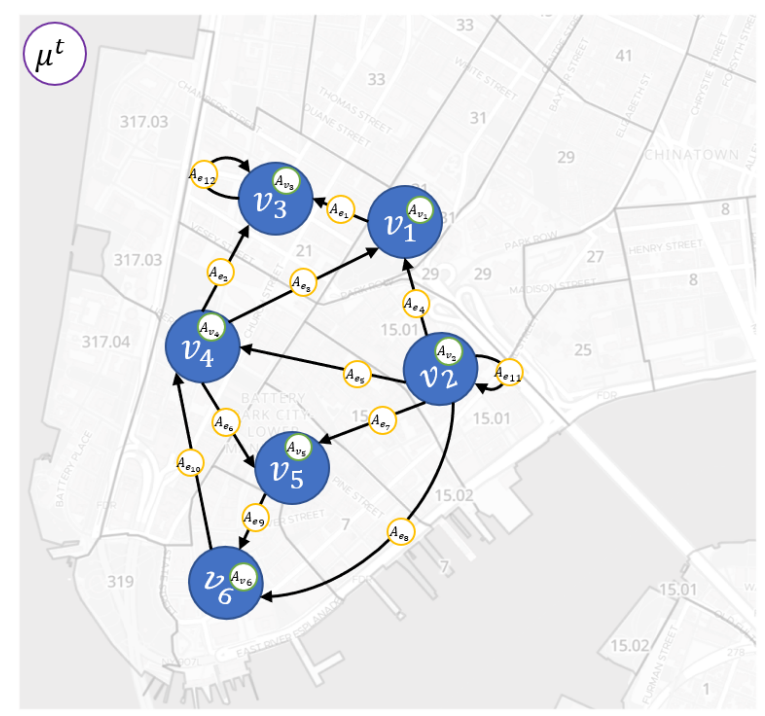

Figure 3.1: A depiction of our city representation with associated attributes.

\subsection{Trip Demand Prediction Problem}

In the context of our city representation, a trip request is expressed by three components: a pickup region, a drop-off region, and a time interval at which the trip occurs. 
We divide a day into a fixed number of time intervals. We denote the total number of time intervals for the entire dataset (e.g., one year of data records) by T. Further, we define trip demand as the number of trip requests that occur between two regions (i.e., on an edge) per time interval. We denote the trip demand on edge $e$ at time interval $t$ by $q_{e}^{t}$. Then, we let $q^{t}$ be the set of all trip demand, $\left\{q_{0}^{t}, \ldots, q_{|E|-1}^{t}\right\}$, for the graph at a given time interval $t$.

\subsubsection{Problem Definition}

We formulate the trip demand prediction problem as a time-series trip forecasting task. Trip forecasting infers future trip demand based on past observations of actual trip records and global data. We define the time-dependent city observation as $s^{t}$, where $t$ is a time interval and $s^{t}=\left(q^{t}, \mu^{t}\right)$.

The forecasting problem aims at learning a function $\mathcal{F}(\cdot)$ that can infer from a sequence of historical city observations future trip demand. The formulation of $\mathcal{F}(\cdot)$ is computed as

$$
\hat{q}^{t+1}=\mathcal{F}\left(\left\{s^{t-\omega+1}, \ldots, s^{t}\right\}, G(V, E)\right),
$$

where $\omega$ is a look-back window size hyperparameter of the model, also referred to as length of sequence or time steps in the literature. The $\hat{q}^{t+1}$ is the predicted trip demand, opposed to $q^{t+1}$, which is the ground truth. In addition, another objective of the forecast problem is to generalize the prediction to alternative city graph structures by adjusting the weight parameters of the supervised learning algorithms used within the function $\mathcal{F}(\cdot)$. 


\subsection{CityNet Model}

In this section, we present our proposed City Graph Neural Network (henceforth CityNet) Model for accomplishing the trip forecasting task. Computation in the network is divided into two submodels node focused and edge focused. The node focused submodel learns the dynamic temporal dependence of ride-hailing trip data, including its complex relationship on demographic, socio-economic, and landuse factors (detailed in Section 3.3.1). The edge focused submodel adaptively models the correlation between the origin and destination regions in order to predict trip demand, which is followed with trip request generation (detailed in Section 3.3.2). Algorithm 3.1 summarizes the process of both submodels. Finally, in Section 3.3.3 we detail the learning procedure used to train the entire CityNet Model.

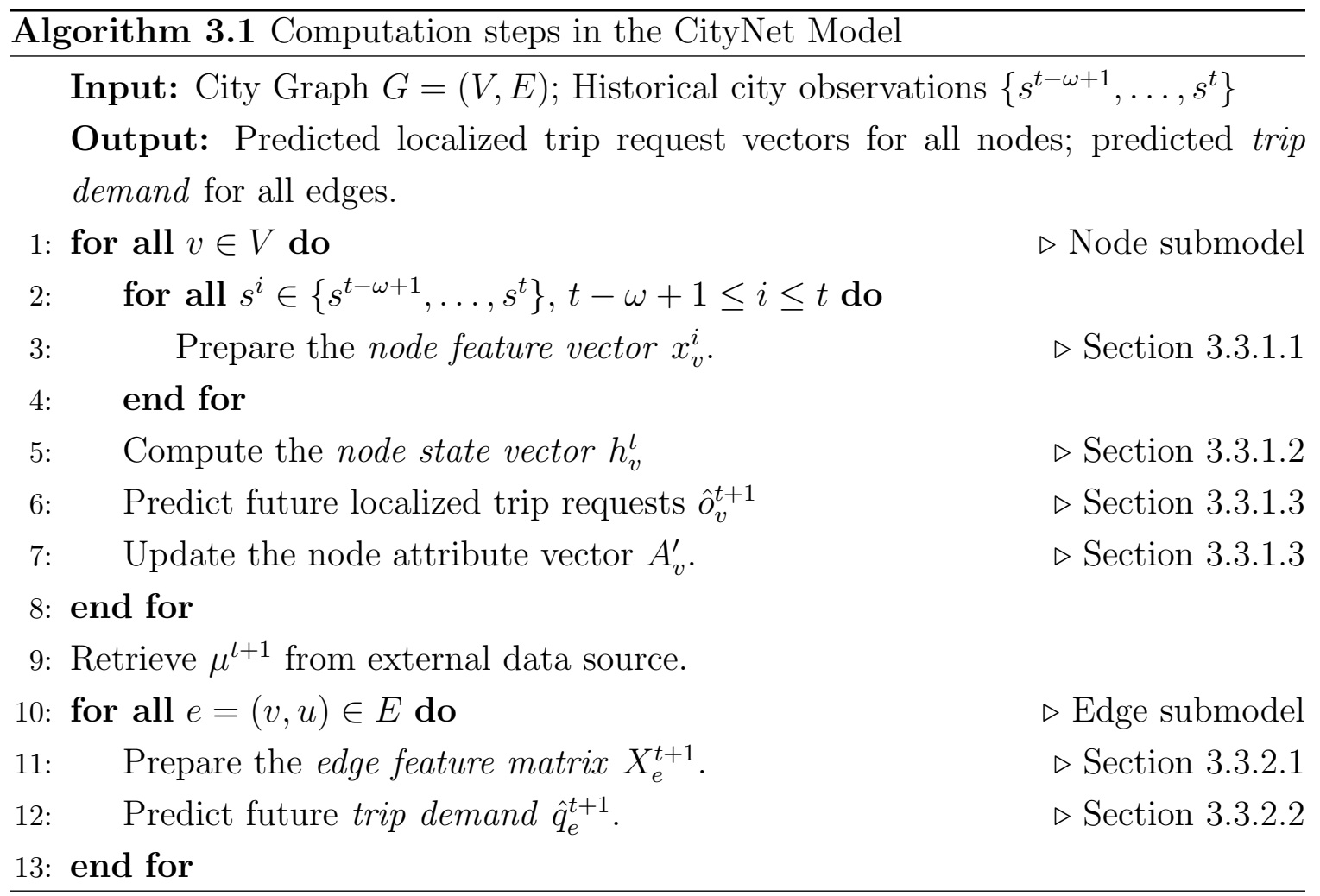


Before continuing further into different subsections, we summarize the notations used throughout this chapter. The notations and their detailed description can be found in table 3.1.

\subsubsection{Node Submodel}

The purpose of the node submodel is to update the node attribute vectors with upcoming localized trip request values. For every node $v \in V$, we execute three main computation steps to update the node attributes. First, we perform node feature preparation to construct a feature vector (Section 3.3.1.1). Then, we process the features with a machine learning algorithm to generate a node state vector (Section 3.3.1.2). Lastly, we predict localized trip request values and append them to the node attributes (Section 3.3.1.3). The updated attributes are later use for trip demand prediction on the edges.

\subsubsection{Node Feature Preparation}

At time interval $t$, the model receives a sequence of historical city observations in chronological order, $\left\{s^{t-\omega+1}, \ldots, s^{t}\right\}$. For each city observation $s^{i} \in\left\{s^{t-\omega+1}, \ldots, s^{t}\right\}$, where $t-\omega+1 \leq i \leq t$, we execute the node feature preparation function, NodeFeaturePrep. For a node $v$, the function takes $s^{i}$ and the node attribute vector $A_{v}$ as input and outputs a node feature vector. We begin the process by extracting the trip demand related to the node. An example of trip extraction is illustrated on the left side of Figure 3.2. We then aggregate the trip demand from all adjacent edges of the node. The aggregation is conducted separately for incoming and outgoing edges. 
Table 3.1: General notations and symbols used in this chapter

\begin{tabular}{l|l}
\hline Notation & Description \\
\hline$G$ & City graph structure, where $G=(V, E)$ \\
$V$ & Set of nodes in $G$ of cardinality $n$ \\
$E$ & Set of edges in $G$ of cardinality $n^{2}$ \\
$I N(v)$ & Set of edges incoming to node $v$ \\
$O U T(v)$ & Set of edges outgoing from node $v$ \\
$A_{v}$ & Node attribute vector of size $\alpha^{V}$ \\
$A_{e}$ & Edge attribute vector of size $\alpha^{E}$ \\
$T$ & Number of time intervals \\
$\mu^{t}$ & Global attribute vector at time interval $t$ of size $\alpha^{\mu}$ \\
$q^{t}, \hat{q}^{t}$ & Actual and predicted trip demand set at time interval $t$ \\
$q_{e}^{t}, \hat{q}_{e}^{t}$ & Actual and predicted trip demand on edge $e$ at time interval $t$ \\
$s^{t}$ & where $q_{e}^{t} \in q^{t}$ \\
$\omega$ & City observation at time interval $t$, where $s^{t}=\left(q^{t}, \mu^{t}\right)$ \\
$x_{v}^{t}$ & Look-back window size hyperparameter \\
$X_{e}^{t}$ & Feature vector for node $v$ at time interval $t$ \\
$h_{v}^{t}$ & Feature matrix for edge $e$ at time interval $t$ \\
$m_{v}^{t}$ & State vector for node $v$ at time interval $t$ \\
$W_{f}, U_{f}$ & Node representation vector for node $v$ at time interval $t$ \\
$b_{f}$ & Weight matrices used in a function $f$ (trainable parameters) \\
$\sigma$ & Bias vector used in a function $f$ (trainable parameter) \\
$\tanh$ & Logistic sigmoid function \\
$\otimes$ & Hyperbolic tangent function \\
$\oplus$ & Element-wise product operator \\
$*$ & Concatenation operator \\
$\theta$ & Convolution product operator \\
$\phi$ & Set of all trainable parameters in RNN \\
\hline & Set of all trainable parameters in CNN \\
\hline
\end{tabular}




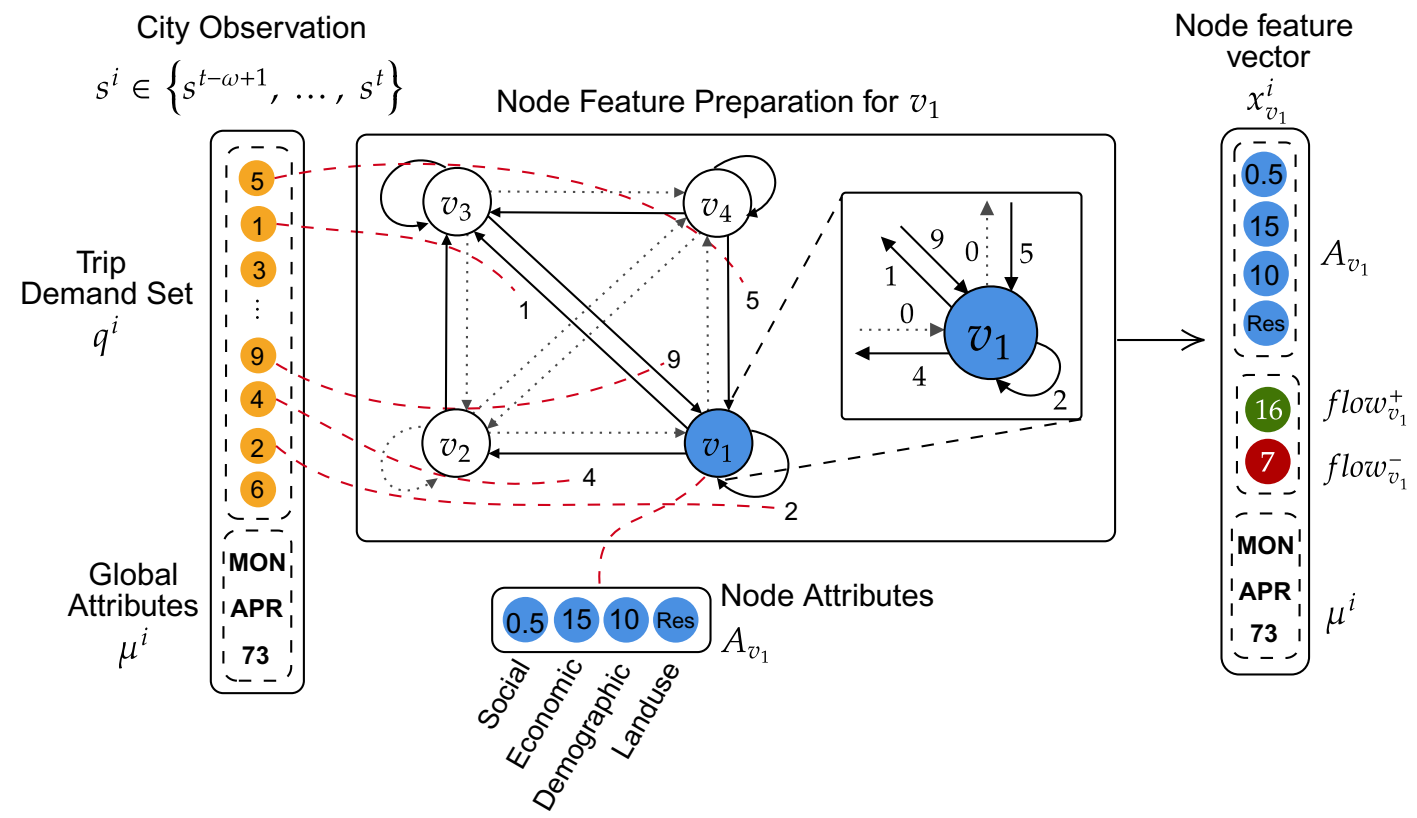

Figure 3.2: Illustration of the node feature preparation step. The process extracts the trip demand related to the selected node, illustrated in blue, and assigns them to the corresponding edges. Note, edges with 0 trip value are dotted out to signify that they are ignored during computation. The process then computes the flow ${ }^{+}$ and flow $^{-}$for the selected node, and then constructs the feature vector. All values depicted in the figure are an example.

Specifically, the following summations are applied:

$$
f l o w_{v}^{-}\left(q^{i}\right)=\sum q_{e}^{i}, \forall e \in O U T(v)
$$

where flow $w_{v}^{-}$is the number of outgoing trip requests that designate node $v$ as the origin of a trip, and

$$
\operatorname{flow}_{v}^{+}\left(q^{i}\right)=\sum q_{e}^{i}, \forall e \in I N(v)
$$

where flow $_{v}^{+}$is the number of incoming trip requests that designate node $v$ as the destination of a trip. Both incoming and outgoing trip values are considered as 
localized representations of trip demand for a region.

We then construct a feature vector by appending the node attributes to the global attributes, in addition to the incoming and outgoing trip request values, as illustrated on the right side of Figure 3.2. The feature vector for node $v$ is denoted as

$$
x_{v}^{i}=\left[A_{v}, \mu^{i}, \operatorname{flow}_{v}^{-}\left(q^{i}\right), \operatorname{flow}_{v}^{+}\left(q^{i}\right)\right] .
$$

\subsubsection{Node State Computation}

In this step, we generates a node state vector by modelling the multivariate and sequential dependency present in the node feature vectors. For our work, we use the GRU architecture due to its relative performance gains and computational speed over other recurrent models, as found by $[66,67]$.

Figure 3.3 illustrates the architecture of the GRU during an internal time step $j$, where the reset gate is denoted by $r^{j}$ and update gate by $z^{j}$.
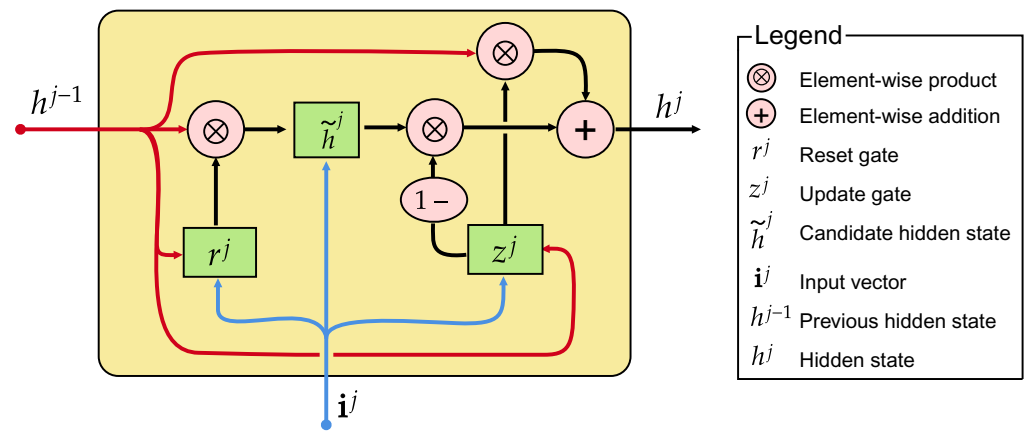

Figure 3.3: GRU Cell Architecture 
The reset and update gates are computed as follows:

$$
\begin{aligned}
& r^{j}=\sigma\left(W_{r} \mathbf{i}^{j}+U_{r} h^{j-1}+b_{r}\right), \\
& z^{j}=\sigma\left(W_{z} \mathbf{i}^{j}+U_{z} h^{j-1}+b_{z}\right),
\end{aligned}
$$

where $\sigma$ is the sigmoid function and $\mathbf{i}^{j}$ is the input vector. $W_{r}$ and $U_{r}$, as well as $W_{z}$ and $U_{z}$ are trainable weight matrices, while $b_{r}$ and $b_{z}$ are trainable bias vectors specific to the reset and update gates, respectively. The hidden state $h^{j}$ is then computed by

$$
h^{j}=z^{j} \otimes h^{j-1}+\left(1-z^{j}\right) \otimes \tilde{h^{j}},
$$

where $\tilde{h^{j}}$ is the candidate hidden state, $\otimes$ is the element-wise product (Hadamard product) operator. The initial hidden state vector, $h^{\text {initial }}$, is initialized using a glorotuniform distribution [68]. Finally, $\tilde{h^{j}}$ is formulated as

$$
\tilde{h^{j}}=\tanh \left(W_{h} \mathbf{i}^{j}+U_{h}\left(r^{j} \otimes h^{j-1}\right)+b_{h}\right),
$$

where tanh is the hyperbolic tangent function. $W_{h}$ and $U_{h}$ are trainable weight matrices, and $b_{h}$ is a trainable bias vectors. The process of updating all trainable parameters is mentioned in Section 3.3.3. Note, we use the same implementation and activation functions, tanh and $\sigma$, as detailed by by Cho et al. in [52].

We iteratively apply equations $3.5,3.6$ and 3.7 on all $x_{v}^{i} \in\left\{x_{v}^{t-\omega+1}, \ldots, x_{v}^{t}\right\}$ to update the hidden state vector of the GRU, where the last hidden state, denoted by $h_{v}^{t}$, is the node state vector. 


\subsubsection{Localized Prediction \& Updating Node Attributes}

From the node state vector we infer upcoming localized (i.e., incoming and outgoing) trip request values, denoted by $\hat{o}_{v}^{t+1}$, by performing linear regression. We use a single dense neural layer, where the output vector contains two scalars and is computed by:

$$
\hat{o}_{v}^{t+1}=\left\{f \hat{l o w} w_{v}^{+}, f \hat{l o w} w_{v}^{-}\right\}=W_{o} h_{v}^{t}+b_{o} .
$$

The $f \hat{l o w} w_{v}^{+}$and $f \hat{l o w} w_{v}^{-}$values signify the predicted incoming and outgoing trip demand, respectively, for node $v$ for the upcoming time interval. $W_{o}$ is a trainable weight matrix and $b_{o}$ is a trainable bias vector. We point out that the ground truth localized trip requests set is given by $o^{t+1}, \forall v \in V$. We illustrate the complete GRU network in Figure 3.4. Note, the parameter values used in the figure were empirically found to give the best performance for our dataset, which we discuss further in Chapter 4. The final part of the node submodel is updating the node attributes. We append the node attribute vector with the predicted localized trip requests vector as follows:

$$
A_{v}^{\prime}=\left[A_{v}, \hat{o}_{v}^{t+1}\right],
$$

where $A_{v}^{\prime}$ is the updated node attribute vector.

\subsubsection{Edge Submodel}

Before we execute the edge submodel computation, we obtain the global attribute

data for the upcoming time interval, $\mu^{t+1}$, by calling an external data source with the desired time interval. Note that simple global data, such as the date and time 


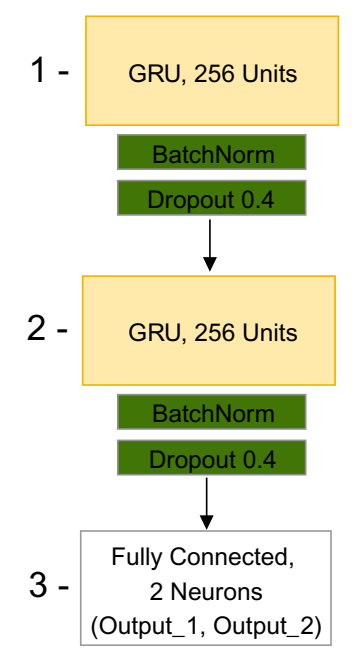

Figure 3.4: Illustration of the full node submodel neural network, where output_1 is the predicted incoming trip value and output_2 is the predicted outgoing trip value.

information, can be precomputed and readily accessed. However, complex global data, such as meteorological information, is provided by querying an external system like a weather forecasting station.

Further, for every edge $e \in E$, we use a Convolutional Neural Network (CNN) to infer trip demand. To use CNNs for pattern recognition the input data needs to be organized such that the network can capture embedded information between the data elements. It is intuitive for "image" data to be arranged as a two-dimensional matrix (with three channels for RGB colors), where the convolution operation can extract feature maps between neighboring pixels. In this work, we propose a novel approach to apply CNNs for trip demand prediction. We leverage the convolution operation to find patterns between the attributes of a pair of nodes. In other words, for a given edge, we stack the pairwise node attribute vectors to form a two-dimensional matrix (with a single channel). We describe the process further in the following section. 


\subsubsection{Edge Feature Preparation}

We perform edge feature preparation by taking $\mu^{t+1}$ and the edge attribute vector $A_{e}$ as input, as well as the updated node attribute vectors for the pair of nodes adjacent to the edge. We output a two-dimensional edge feature matrix, denoted by $X_{e}^{t+1}$. The process begins by aggregating both node attribute vectors to the edge as illustrated in Figure 3.5. Specifically, for an edge $e=(v, u)$, the aggregation procedure results in the following initial matrix:

$$
\bar{X}_{e}^{t+1}=\left[\begin{array}{c}
A_{v}^{\prime} \\
A_{u}^{\prime}
\end{array}\right] .
$$

We then append the edge attributes and global attributes to the initial matrix,

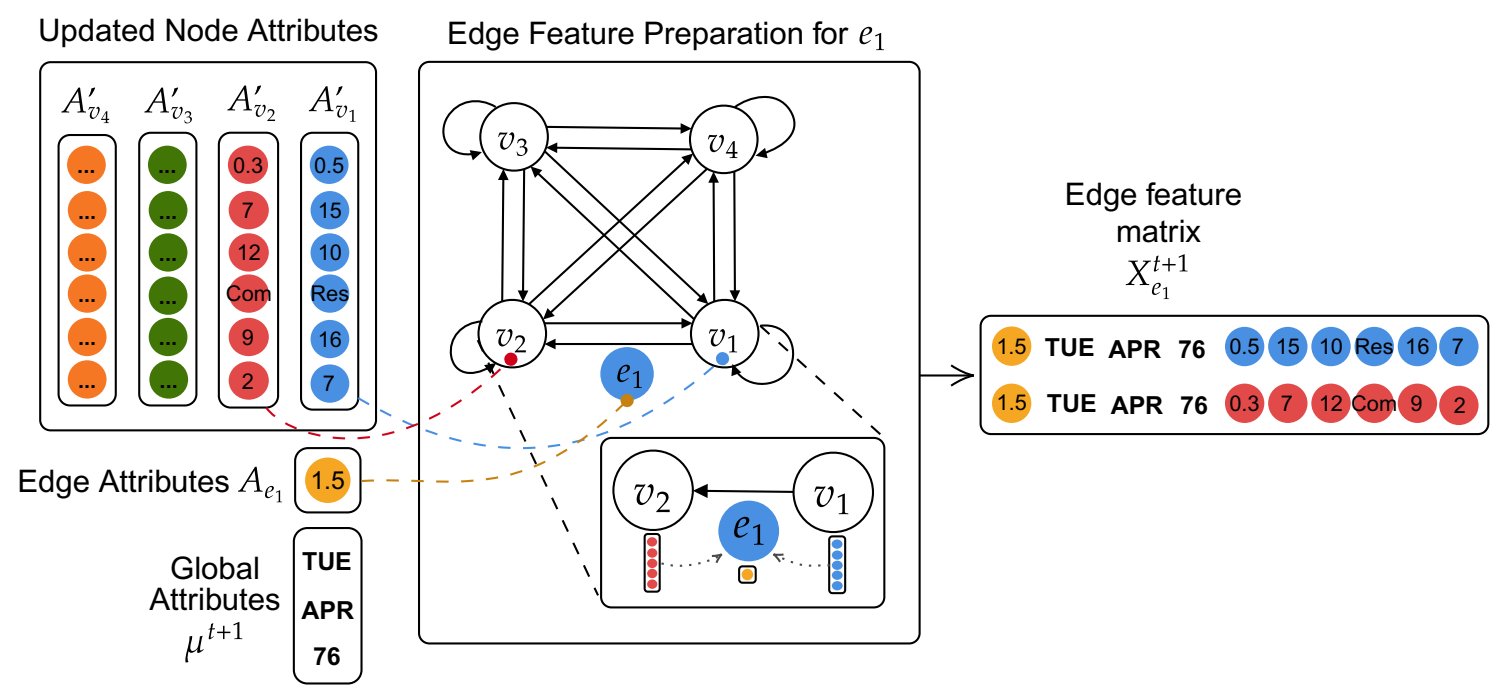

Figure 3.5: Edge Feature Preparation: The function propagates the node representation vectors to the selected edge, illustrated in blue. It then outputs the feature matrix by concatenating the edge attributes and global attributes to the result of the aggregation. All values depicted in the figure are an example. 
resulting in the edge feature matrix:

$$
X_{e}^{t+1}=\left[\begin{array}{cc}
A_{e} & \mu^{t+1} \\
A_{e} & \mu^{t+1}
\end{array}\right] \oplus\left[\begin{array}{l}
A_{v}^{\prime} \\
A_{u}^{\prime}
\end{array}\right]=\left[\begin{array}{ccc}
A_{e} & \mu^{t+1} & A_{v}^{\prime} \\
A_{e} & \mu^{t+1} & A_{u}^{\prime}
\end{array}\right]
$$

where $\oplus$ denotes the concatenation operator. For clarity we note that the edge attributes and global attributes are repeated once for each row. Additionally, we refer to the first row as the origin row and the second row as the destination row. The main purpose of engineering the edge feature matrix in this form is to allow the convolutional operations to extract patterns between the origin row and destination row, imposing an implicit relationship similar to that of pixels in an image. However, in our experiments, we found no spatial significance in the order of the attributes beyond aligning them to coincide.

\subsubsection{Trip Demand Prediction}

The final step of the CityNet model is to forecast trip demand on the edges of the graph. As mentioned previously, we implement this step using the CNN architecture. The convolutional operation is computed by:

$$
\operatorname{conv}=\operatorname{ReLU}\left(W_{c} * \mathbf{I}+b_{c}\right),
$$

where $\mathbf{I}$ is an input matrix, $W_{c}$ is a trainable kernel matrix, $b_{c}$ is a trainable bias vector, and $*$ denotes the convolutional product operator. The $R e L U$ is the Rectified Linear Unit activation function, which is used for all layers in the network. Our network design relies on four fundamental operations, illustrated in Figure 3.6 and 
annotated as follows:

1. Several convolution layers with kernel size $1 \times 3$ : The network can learn detailed feature maps from the origin and destination rows separately. This allows for important details to carry over while suppressing others leading to complex pattern recognition on higher convolutional layers.

2. Single convolution layer with kernel size $2 \times 3$ : This is the main operation which allow the network to extract patterns between the origin and destination rows. This operation will reduces the two-dimensional edge feature matrix to a single dimension, which represents the correlation between the rows.

3. Additional convolution layers with kernel size $1 \times 3$ : This operation yields highly expressive feature maps that can be used for predication.

4. Average pooling layer with kernel size $1 \times 2$ : This performs down-sampling by a factor of 2 , where the output is the average value of each patch. This results in condensed feature maps. We conjecture that our results are invariant to the arrangement of columns in the input matrix due to the average pooling operation.

Based on empirical evaluation of the kernels' size, we found no significant improvement in error rate by increasing the dimension width to 4 or 5 in all respective convolution operations. We also did not see any notable improvement by decreasing the dimension width to 2, only increased computation time. Furthermore, throughout the CNN we maintain a stride of 1 with no padding for all convolution and pooling layers. 


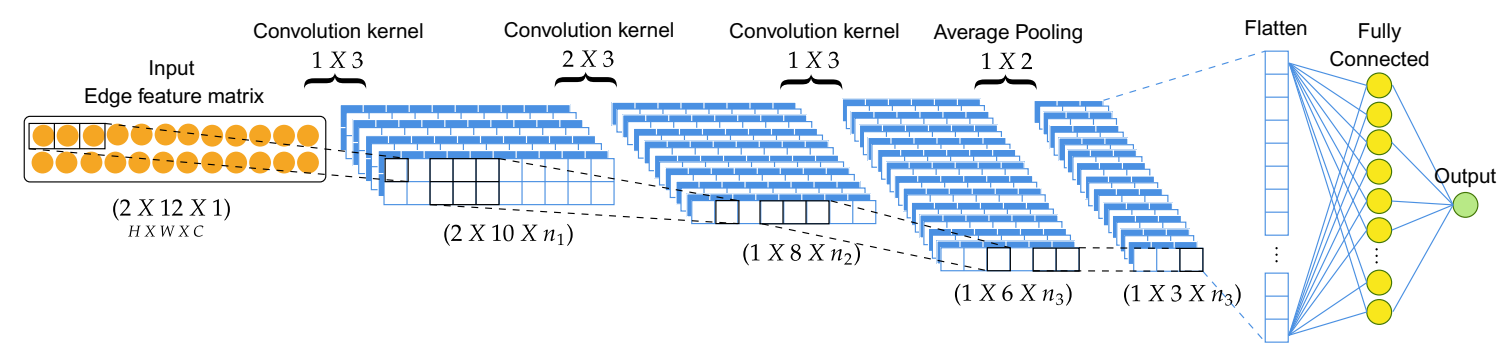

Figure 3.6: Illustration of CNN operations in our network. The $n_{1}, n_{2}$ and $n_{3}$ are the number of kernels used for each respective layer.

Demand Prediction The last layers of the CNN predicts trip demand by learning non-linear combinations on the feature maps resultant from the output of the convolutional layers. We implement this step using several fully-connected (FC) neural layers, where the final output layer performs linear regression on the high-level features. The output, $\hat{q}_{e}^{t+1}$, is a single scaler value that represents the inferred number of trips on the edge $e$ at time $t+1$. The FC layers and the last output layer are formulated as:

$$
\begin{array}{r}
F C=\operatorname{Re} L U\left(W_{f c} \mathbf{i}+b_{f c}\right), \\
\hat{q}_{e}^{t+1}=W_{q} \mathbf{i}+b_{q},
\end{array}
$$

where $\mathbf{i}$ is an input vector, $W_{f c}$ and $W_{q}$ are trainable weight matrices, and $b_{f c}$ and $b_{q}$ are trainable bias vectors. Based on empirical evaluation of several CNN models (detailed in Chapter 4), we found that the layer setup in Figure 3.7, which adheres to the previously mentioned fundamental operations, resulted in the highest performance.

Trip Request Generation We use the predicted trip demand values on the edges to generate theoretical trip requests that can be utilized by ride-hailing, ride-sharing and taxi dispatch systems for demand anticipation. We set the pickup/drop-off location of the trips using a uniform distribution over a region's main road segments 


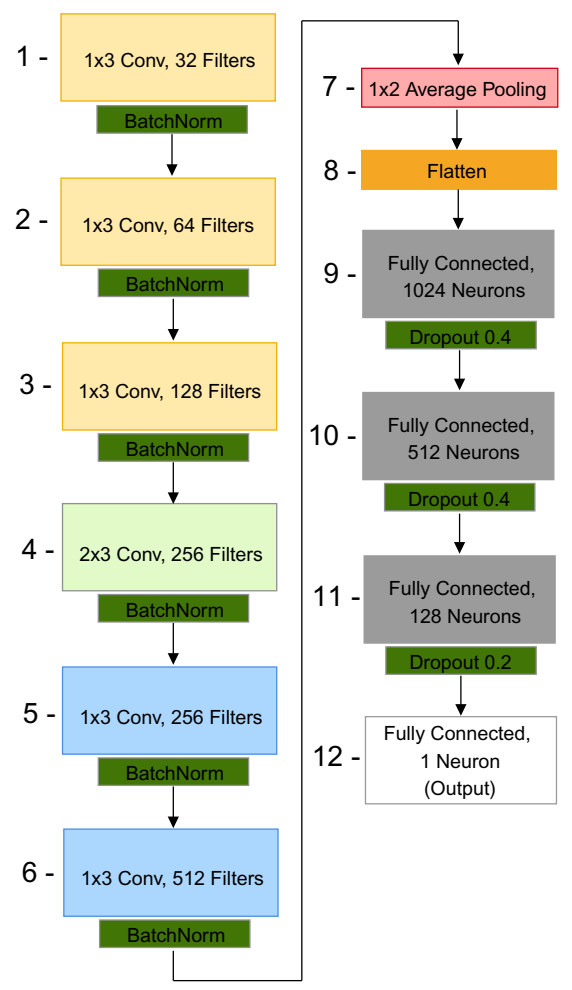

Figure 3.7: An illustration of all the layers in the CNN model. The output is the predicted trip demand.

(not including highways). We also use a uniform distribution of pickup timestamps within each time interval.

\subsubsection{Learning Algorithm}

Here we detail the learning pipeline used to train the machine learning algorithms used in the node submodel and edge submodel segments of the CityNet model. We train the neural networks using a supervised learning approach. The goal of the training process is to minimize a loss function between the predicted and target output of the model by adjusting the network's trainable parameter until a desirable state is 
achieved.

Before we begin training the neural networks we setup the training dataset. First, we define $S$ as the set of all city observations $\left\{s^{0}, \ldots, s^{T}\right\}$. Then, we build the training data graphs by iterating over all time interval in our dataset $t: 0 \rightarrow T$. For each $t$, we append a tuple to the training set, which consists of a sequential subset of city observations, denoted by $S^{t}\left(\left|S^{t}\right|=\omega\right)$, as well as the target labels for the node and edge submodels. We summarize the steps of constructing the training set in algorithm 3.2.

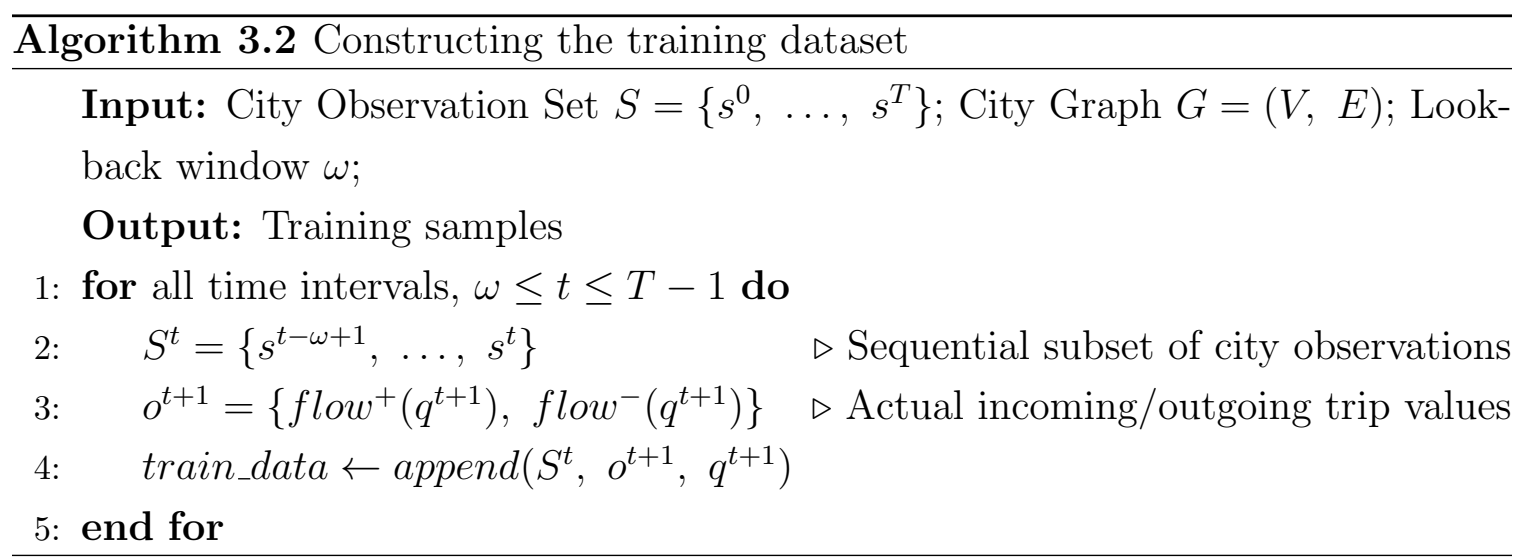

\subsubsection{Training the Node Submodel}

We begin by training the node submodel. Specifically, we jointly train the GRUs and dense neural layer using the following loss function:

$$
\mathcal{L}(\theta)=\lambda * \sqrt{\frac{1}{n} \sum_{i=1}^{n}\left(\hat{y}_{i}-y_{i}\right)^{2}}+\frac{1}{n} \sum_{i=1}^{n}\left(\log _{e}\left(\hat{y}_{i}+1\right)-\log _{e}\left(y_{i}+1\right)\right)^{2},
$$

where $\theta$ represents the set $\left\{W_{r}, U_{r}, b_{r}, W_{z}, U_{z}, b_{z}, W_{h}, U_{h}, b_{h}, W_{o}, b_{o}\right\}$ of trainable parameters. $\lambda$ is a hyperparameter in $[0,1), n$ here is the number of samples, $\hat{y}_{i}$ is the 
predicted value of the $i^{t h}$ sample and $y_{i}$ is the corresponding true value. We note that the loss function $\mathcal{L}$ consists of two parts, Mean Squared Logarithmic Error (MSLE) loss, plus a fraction of Root Mean Squared Error (RMSE) loss. Empirically, we found that using a two part loss function allowed us to utilize the properties of both metrics. First, a compelling property of the squared logarithmic error is that it penalizes underestimation more than overestimation (for the same truth value). This property is desirable since we expect our model to overestimate its predictions to meet the minimum number of trip demand and buffer against demand surge. Second,

we empirically found that adding a fraction of RMSE gives us the best fit results to the ground truth values.

Further, we perform gradient descent to minimize the loss functions with respect to $\theta$. We carry out the optimization process using Stochastic Gradient Decent (SGD) with momentum. A single update of the parameters is formulated as:

$$
\begin{aligned}
\mathbf{u}^{j} & =\gamma \mathbf{u}^{j-1}+\eta \frac{\partial \mathcal{L}(\theta)}{\partial \theta}, \\
\theta^{\prime} & =\theta-\mathbf{u}^{j}
\end{aligned}
$$

where $\mathbf{u}^{j}$ is an update vector, $j$ is an internal time step, $\eta$ is the learning rate hyperparameter, $\gamma$ is the momentum hyperparameter, and $\theta^{\prime}$ is the adjusted values for the trainable parameters. Algorithm 3.3 outlines the training pipeline for the node submodel. We cease the training process after a fixed number of iterations, or no improvement is recorded on the error loss. 


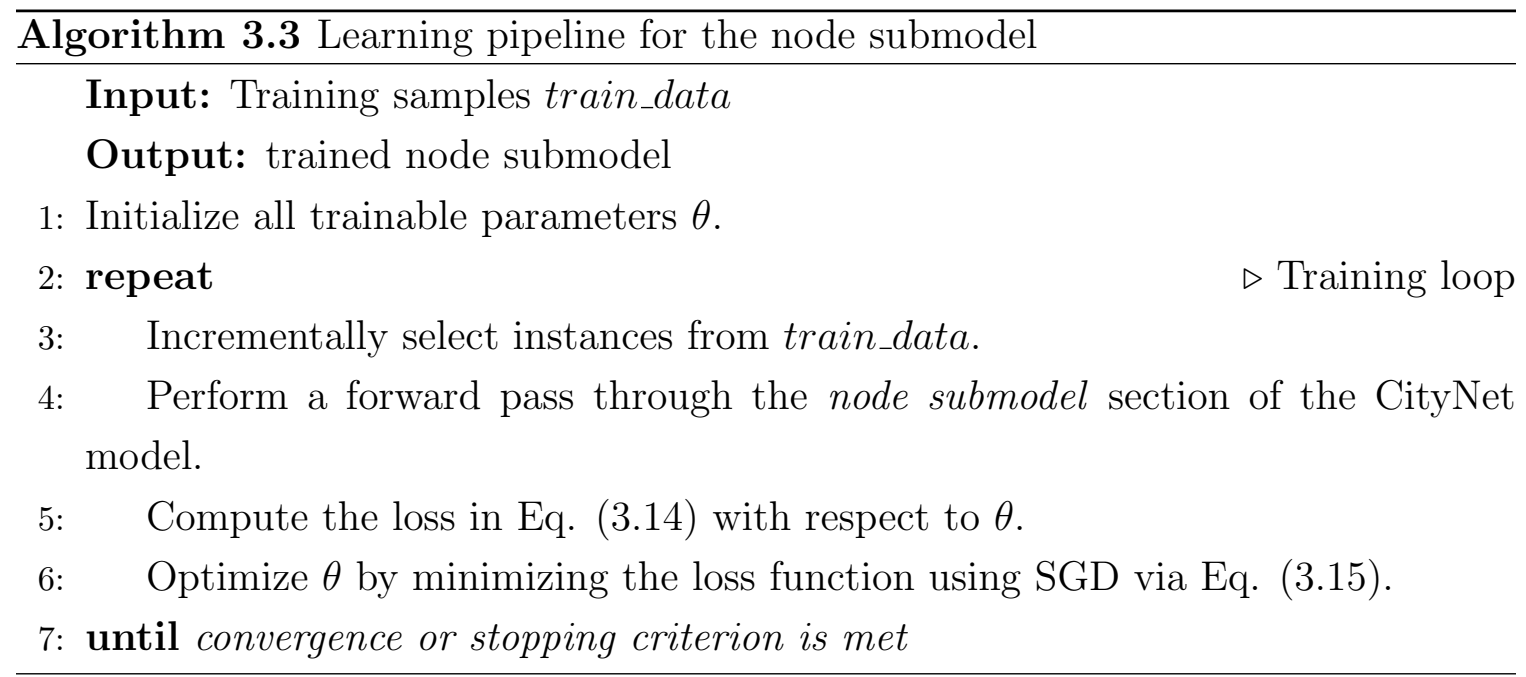

\subsubsection{Training the Edge Submodel}

Through empirical evaluation, we found that training the node submodel to completion yields more stable performance for the edge submodel when compared to jointly training the two submodels. We compare and discuss the results in Chapter 4 .

We train the CNN using the same loss function as equation 3.14. However, we use $\phi$ to represent all trainable parameters, $\left\{W_{c}, b_{c}, W_{f c}, b_{f c}, W_{q}, b_{q}\right\}$, in the network. We also carry out the optimization process using SGD with momentum, similar to the node submodel. A single update of the parameters is formulated as:

$$
\begin{aligned}
\mathbf{p}^{j} & =\gamma \mathbf{p}^{j-1}+\eta \frac{\partial \mathcal{L}(\phi)}{\partial \phi}, \\
\phi^{\prime} & =\phi-\mathbf{p}^{j}
\end{aligned}
$$

where $\mathbf{p}^{j}$ is an update vector, and $\phi^{\prime}$ is the adjusted values for the trainable parameters. Algorithm 3.4 outlines the training pipeline for the edge submodel. We also terminate the training process for the edge submodel after a fixed number of 
iterations, or no improvement is recorded on the error loss.

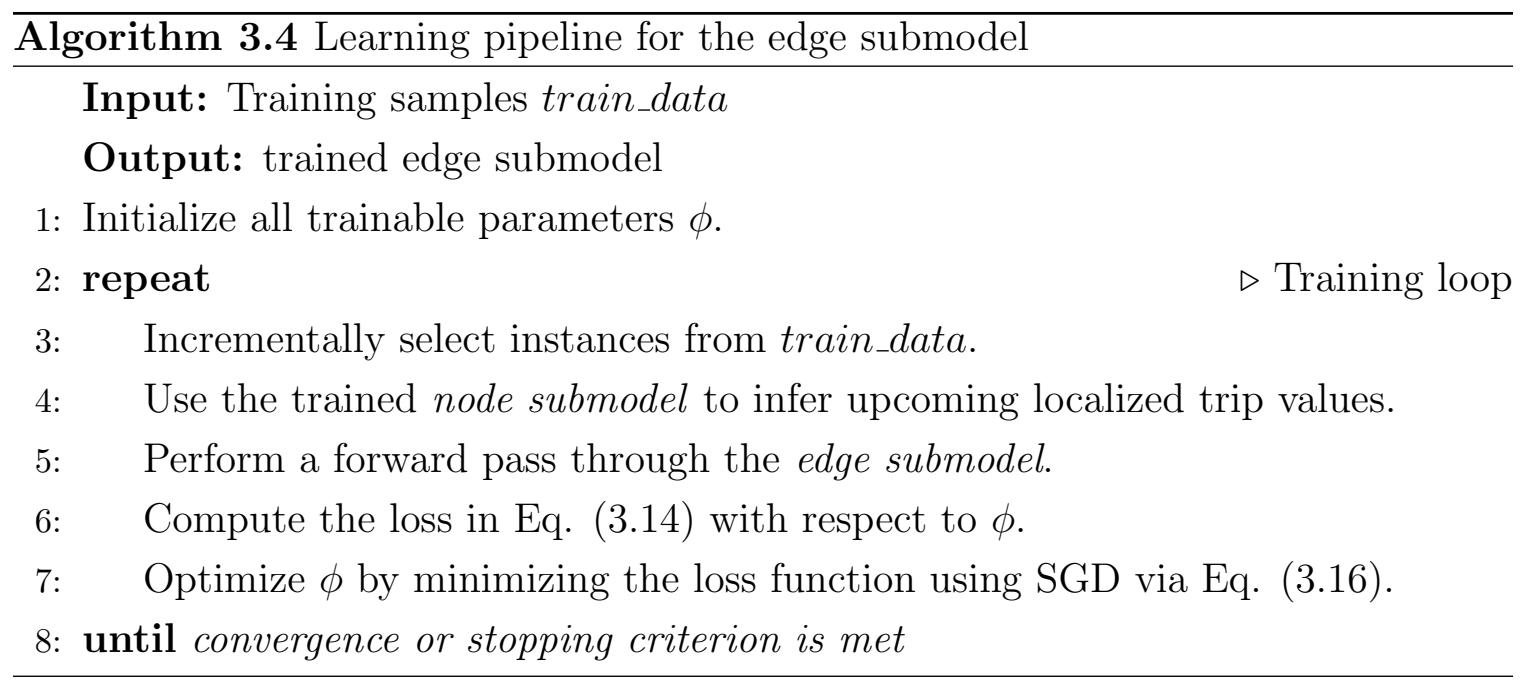




\section{Chapter 4}

\section{Experimental Results and Analysis}

For our experiments we chose to represent and utilize the information provided by the city of New York and its agencies. In this chapter, we first introduce the various datasets used in our experiments (Section 4.1), followed by an analysis of the socioeconomic factors that effect trip flow (Section 4.2). We lastly detail the experiment results obtained from various benchmark methods, and different CityNet variants and hyperparameters (Section 4.3).

\subsection{Data Description \& Preparation}

We tabulated observations of trip records and weather data following a predefined set of time segments. We divided a day into 9 intervals (detailed in Table 4.1) based on travel patterns perceived from analyzing trip volume per hour of the day (illustrated 
in Figure 4.1). We selected these time intervals to highlight daily on-peak and offpeak traffic hours. Furthermore, we extracted and used data records from January December 2014 for our experiments, as a proof of concept. We note that due to time and dataset limitations (detailed in Section 4.1.2) we could not include data records beyond the year 2014. Additionally, since we employ data for 365 days, the total number of time intervals, $\mathbf{T}$, is 3285 .

Table 4.1: Time segments used for data collection and processing.

\begin{tabular}{|cc|}
\hline Time Segments & Description \\
\hline \hline $0: 00-4: 59$ & Night \\
\hline $5: 00-6: 59$ & Early Morning \\
\hline $7: 00-8: 59$ & Morning Rush \\
\hline $9: 00-11: 59$ & Morning \\
\hline $12: 00-12: 59$ & Lunch \\
\hline $13: 00-15: 59$ & Afternoon \\
\hline $16: 00-17: 59$ & Afternoon Rush \\
\hline $18: 00-21: 59$ & Evening \\
\hline $22: 00-23: 59$ & Midnight \\
\hline
\end{tabular}

In the following subsections, we detail the city partitioning method, followed by describing the trip records dataset and the datasets used to construct the node, edge, and global attributes. Finally, we detail the process employed to produce city observations for training our CityNet model. We note that all datasets mentioned in this section can be freely retrieved from the NYC Open Data website ${ }^{1}$, and all geographic shapefiles ${ }^{2}$ can be retrieved from the NYC Department of City Planning website ${ }^{3}$.

\footnotetext{
${ }^{1}$ https://data.cityofnewyork.us

${ }^{2}$ Shapefile format is a vector data format that contains the locations, shapes, and features of geographical data used by GIS applications for map building.

${ }^{3}$ https://www1.nyc.gov/site/planning/data-maps/open-data.page
} 


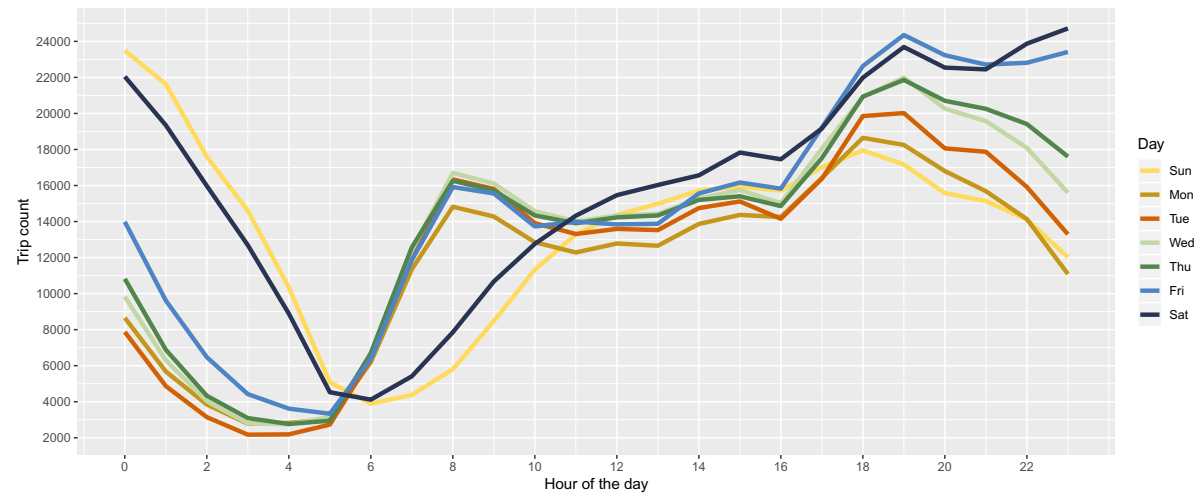

Figure 4.1: Trip volume per hour of the day

\subsubsection{City Partitioning}

For this work, we used cartographic ${ }^{4}$ boundary files - shapefiles - as the bases for constructing the nodes of the city graph. The main requirement was to establish geometries for which census ${ }^{5}$ information and land-use data is naturally available. We considered the following boundary methods: 1) Neighbourhood Tabulation Areas (NTAs), 2) Census Tracts, 3) Census Blocks, and 4) Zoning and Land Use (ZoLa) districts. All methods are illustrated and detailed in table 4.2.

\subsubsection{Selection Criteria}

We evaluated our selected boundary files based on the following metrics: 1) geometric area, 2) number of trips per region per day, 3) Number of no-trip regions and 4) Distance to nearest centroid (measured as the distance between the original position of a trip and the nearest region centroid). We then used the following criterion to

\footnotetext{
${ }^{4}$ Naturally occurring boundaries such as roads, structures, mountains, or bodies of water.

${ }^{5}$ Census is the systematic approach in tabulating information regarding the members of a population.
} 
Table 4.2: Illustration and description of four cartographic boundary files.

\begin{tabular}{|c|c|}
\hline \% & $\begin{array}{l}\text { Neighbourhood Tabulation Areas (NTAs) are } \\
\text { large areas based on city convention for neighbor- } \\
\text { hoods. They divide the city into } 195 \text { regions with } \\
\text { an average population of } 41 \mathrm{k} \text {. Census information } \\
\text { is not specifically tabulated for neighborhood ar- } \\
\text { eas, but can be acquired by aggregating informa- } \\
\text { tion from smaller subdivisions. }\end{array}$ \\
\hline & $\begin{array}{l}\text { Census Tracts are small geographic areas defined } \\
\text { by the Census Bureau to collect and tabulate cen- } \\
\text { sus data. They divide the city into } 2166 \text { tracts } \\
\text { with an average population of } 4000 \text {. Census tracts } \\
\text { are contained within NTAs. }\end{array}$ \\
\hline & $\begin{array}{l}\text { Census Blocks are a subdivision of Census Tracts } \\
\text { and the smallest units by which census information } \\
\text { is collected. There are over 30k blocks within the } \\
\text { city of New York with an average population of } 57 .\end{array}$ \\
\hline 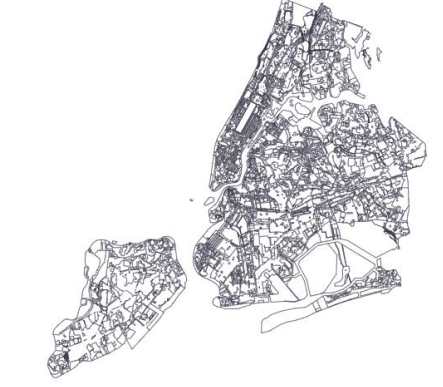 & $\begin{array}{l}\text { Zoning and Land Use (ZoLa) are a categoriza- } \\
\text { tion of plots of land based on designation and con- } \\
\text { textual purpose. They divide NYC into three gen- } \\
\text { eral zoning types (with varying sizes) residential } \\
\text { (R1-R10), commercial (C1-C8), and manufactur- } \\
\text { ing (M1-M3), in addition to } 64 \text { special purpose } \\
\text { zoning types. The total number of regions is } 5206 \text {. }\end{array}$ \\
\hline
\end{tabular}


elect a method with the best possible results to run our experiments on:

- Minimize loss of granularity: Large regions would obscure original trip locations. We observe from Figure 4.2a that NTA regions have an excessive area size, which results in trip requests placed far from their intended locations. This point is further affirmed by Figure 4.2b, where, for NTAs, the average distance of a trip to the nearest centroid location is greater than $2 \mathrm{~km}$. On the other hand, this analysis shows that census blocks have the lowest loss of granularity due to their relatively small area size, which is a desirable quality.

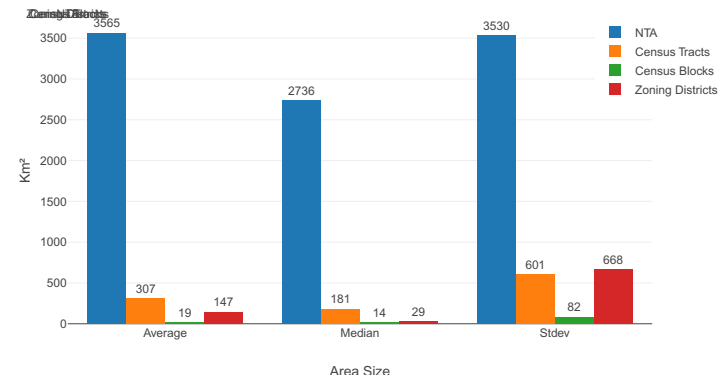

(a) Region area size.

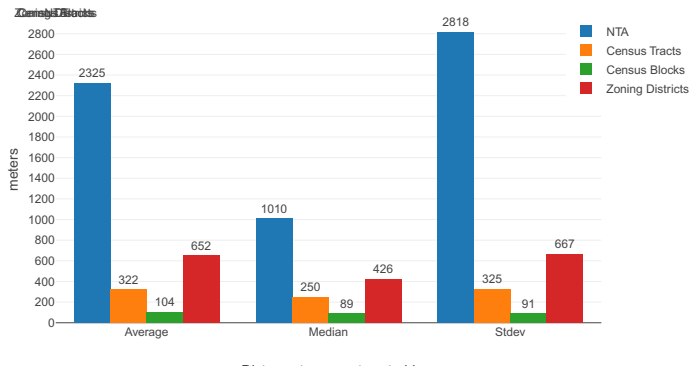

(b) Trip distance to nearest region center.

Figure 4.2: Statistical information regarding city regions and the trips within them.

- Balanced number of trips per region: Regions with a relatively large number of trips, compared to their neighbors (except Airport regions), would result in biased predictions to that region type. Figure 4.3 shows that NTAs exhibit a relatively high average number of trips per region, while census blocks and ZoLa districts display the extreme opposite. The figure also shows that NTAs and census tracts have a suitable median value of trips, 26 and 4 respectively. However, census blocks and ZoLa districts are zero, which is undesirable. 


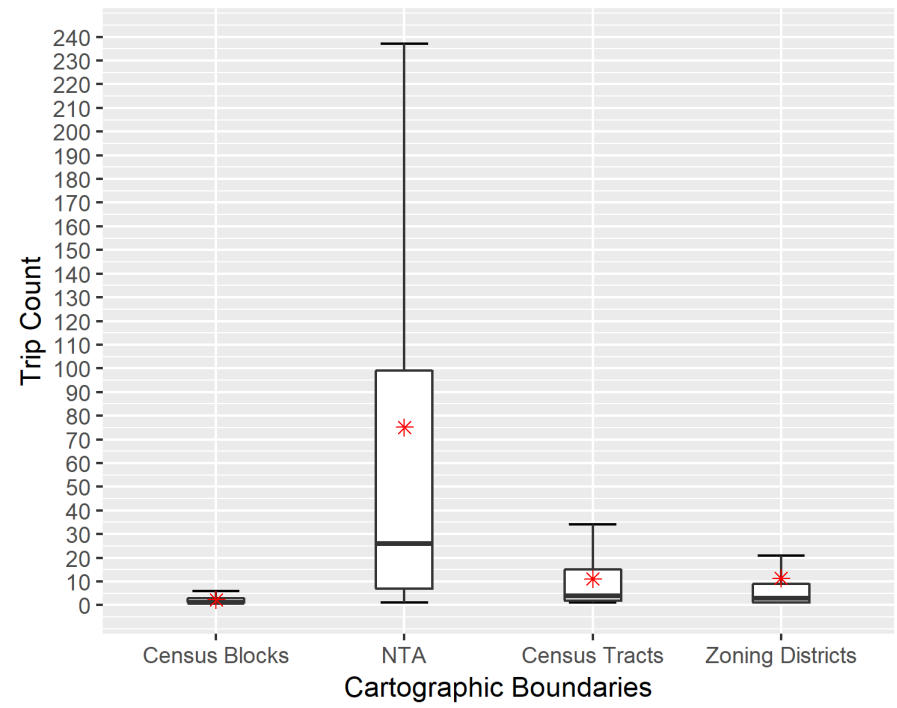

Figure 4.3: Number of trip requests per region. The red star indicates the mean value.

- Minimal number of regions with zero trip requests: Regions with a predominantly inactive trip demand do not provide any insight into the forecasting process. Figure 4.4 shows that $70 \%$ of census blocks and ZoLa districts are inactive regions, which is further illustrated by the median number of trips in Figure 4.3. However, NTAs and census tracts have $20 \%$ of regions with zero trip requests making them an ideal choice.

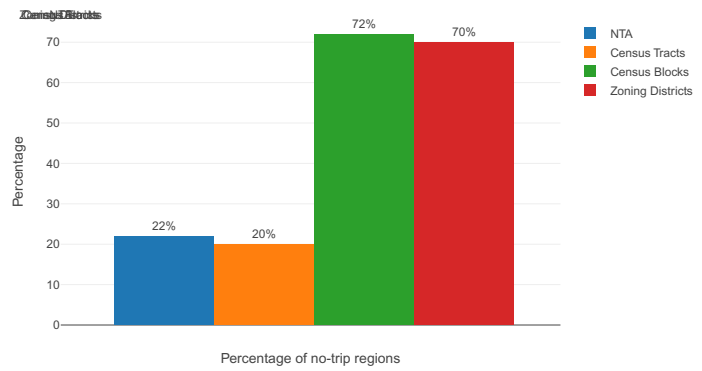

Figure 4.4: Percentage of regions with 0 trip requests 
From our empirical evaluation, we found that census tracts offer the best compromise given our criteria with a small number of regions with zero trip requests, a sufficient number of trips per region, a relatively small area size, and a moderately short trip distance to the nearest centroid. Therefore, we selected Census Tracts as the partitioning method to set up the nodes of the city graph. With the exclusion of Ellis Island, Governors Island, and Staten Island (due to their inaccessibility via direct road network from Manhattan) we obtain 2054 regions before additional processing.

\subsubsection{Land-Use Classification}

Due to census tracts not containing land-use information, we incorporated ZoLa data fields within census tracts. To perform this step, we execute a spatial join operation ${ }^{6}$ (left outer join) between the census tract and ZoLa data fields. As a result, we obtain census tract geometries with 1-or-more ZoLa data fields associated with them. Figure 4.5 depicts the join process. We then use a heuristic (detailed in Appendix A) to label each census tract with a single land-use type based on the associated ZoLa data fields. Regions are then classified based on their respective census tract land-use type in one of the following classes:

- Residential-Small: Low density housing sustaining 1-4 families. They include detached and semi-detached homes, and small apartment houses.

- Residential-Medium: Multi-family housing that include low to mid range apartment buildings. These zones contain small commercial units like convenience stores and restaurants that are part of the facility.

\footnotetext{
${ }^{6}$ Join data fields from one layer to another based on spatial relations (e.g. intersect, contain, overlap) between their geometries.
} 

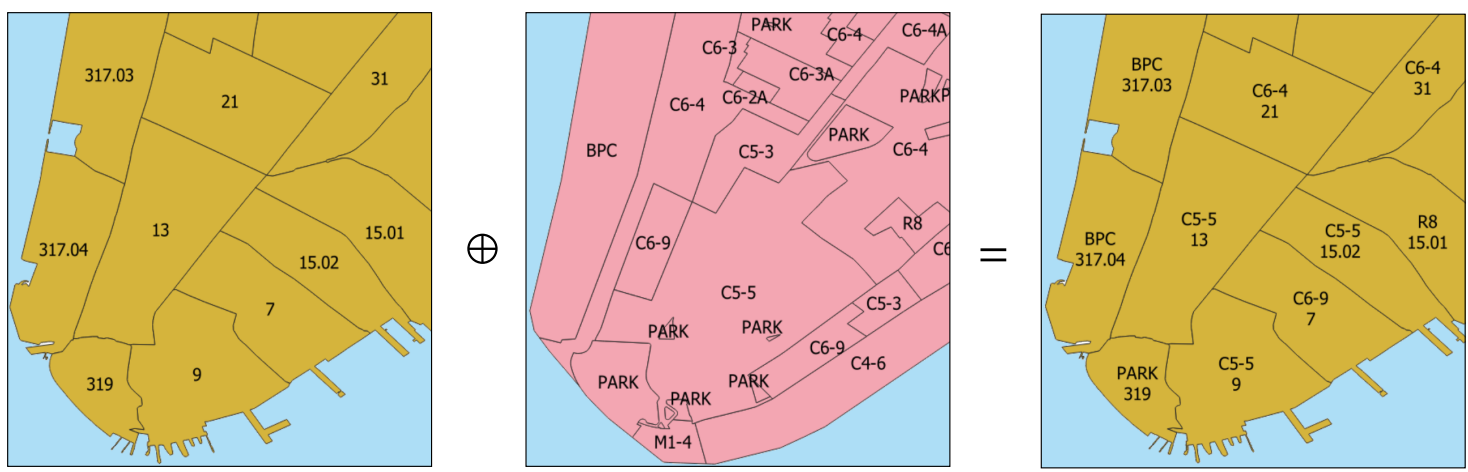

Figure 4.5: Illustration of a spatial join operation between Census tracts and ZoLa geometries, where $\oplus$, here, is the spatial join operand. For clarity, we only show one landuse type per polygon. The resulting geometries are Census Tracts with 1-or-more land-use information.

- Residential-Large: Eight-story or more apartment buildings that also include towers and income-restricted housing units.

- Commercial-Medium: Regional commercial centers covering department stores, theaters, office buildings, and amusement parks.

- Commercial-Large: High-bulk commercial districts encapsulating large hotels, corporate headquarters, and major department store.

- Mixed: These regions contain an equal distribution of small to medium residential and commercial types.

- Airport: Covers the entirety of the airport facility.

- Other: Contains manufacturing areas, parks, playgrounds, and cemeteries. 


\subsubsection{Region Selection}

We sought to select a smaller subset from the 2054 regions to use as nodes for the city graph. We remove all regions classified as Other (61 regions), regions with an annual median number of incoming or outgoing trips less than 2 (1208 regions), and residential regions where the population is zero (3 regions). Empirically, we found that the removed regions do not represent normal traveling patterns around the city. The final region count is 782 , shown in Figure 4.6 in red.

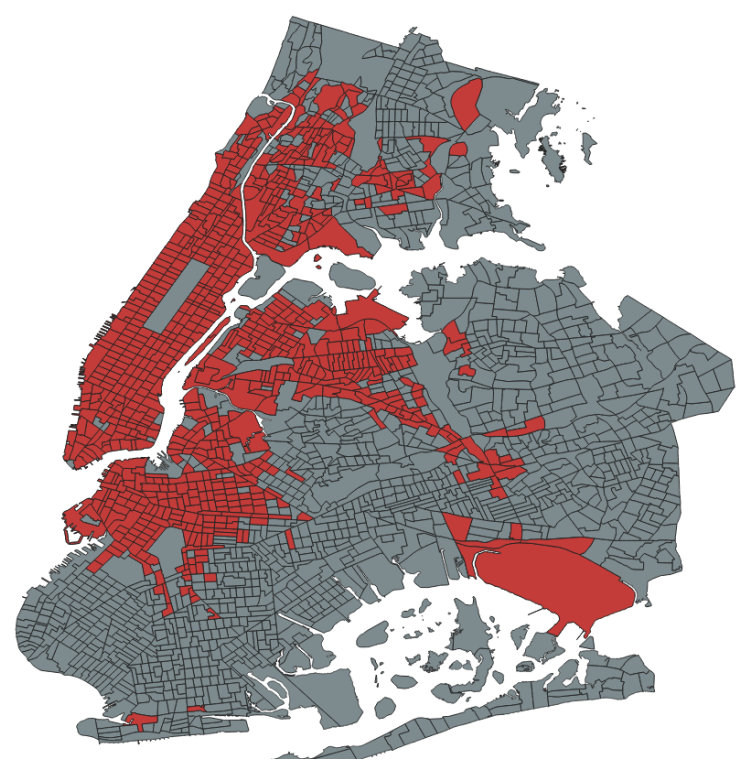

Figure 4.6: Illustration of the final region selection in red.

\subsubsection{Trip Records Dataset}

We utilize the taxi trip data repository provided by the NYC Taxi and Limousine Commission (TLC), which openly provides fulfilled taxi trip records starting from 2009 for all green and yellow taxi-cab vehicles that operate within the city. The 
records are aggregated and reported per month, which contains approximately 14 million taxi records (accumulated annually to $\sim 160$ million records). Each record captures a comprehensive set of information regarding fulfilled trips including, but not limited to, trip pickup/dropoff datetime, pickup/dropoff location in GPS coordinates, as well as trip distance, and fare amount. From each record we extracted the following data fields:

- Pickup latitude/longitude: real number in decimal degrees that represents the geographical location at which the passenger(s) entered the transportation vehicle.

- Dropoff latitude/longitude: real number in decimal degrees that represents the geographical location at which the passenger(s) exited the transportation vehicle

- Pickup timestamp: the date and time instance at which the passenger(s) entered the transportation vehicle.

Before any processing is done on the trip records, we perform data cleaning. We remove all trip records with missing data fields, GPS coordinates that fall beyond the boundaries of the city or at a distance of 100 meters from the nearest road network, as well as records with discrepancies between distance and time traveled. We note that we could not use data from the years 2009 - 2013 as they were missing trip records for long periods of time rendering the dataset incomplete for the year. We also could not use data from July 2016 onward since they do not contain GPS coordinates, which are critical for our current approach. Additionally, we planned to include data records for the year 2015. However, the cleaning process took a significant amount of time and a decision was made to go with year 2014 data alone. 


\subsubsection{Node Attributes}

Each node is associated with four attributes:

- Total Population (Demographic): Number of individuals residing in the region.

- Higher Education (Social): Percentage of the population, 25 years and over, with a bachelor's degree or higher.

- Median Income (Economic): Annual household income within the region.

- Land-use (Categorical): A single land-use type obtained from the labeling heuristic that describes the general purpose of the region.

The attributes are based on information from the 2010 Decennial Census ${ }^{7}$ dataset and the ZoLa data fields. Since each census tract represents a node in our graph, the extraction and assignment of census information is one-to-one. However, as mentioned previously, we use a heuristic to assign a single land-use type from the ZoLa data fields to each node.

\subsubsection{Edge Attributes}

We associate every region pair with the distance on the road network between them. The distance is calculated based on Dijkstra's shortest path algorithm using the regions' centroids. If a region's centroid is inaccessible via a direct road, we realign the centroid to the nearest road segment that minimizes the resulting distance.

\footnotetext{
${ }^{7}$ Decennial census data is collected once every decade. The dataset contains demographic, social, and economic information specific to each census tract. Data from 2010 represents the most recent information.
} 


\subsubsection{Global Attributes}

The global attribute set consists of two types of information weather data and datetime descriptors.

Weather Dataset We retrieve daily weather information from the National Oceanic and Atmospheric Administration website ${ }^{8}$ for the year 2014. The data is collected by a web crawler that parses the information for each year. From each daily reading we extract the following information:

- Average Temperature: Temperature in Fahrenheit degrees.

- Precipitation: Amount of rain fall, snow fall, and ice fall measured in inches.

- Snow Depth: Accumulated snow at ground level measured in inches.

Datetime Information We capture varying granularity of datetime information related to each trip, which is interpreted from the timestamp of the trip record. We record: Time interval, Day of the week, and Month descriptors.

\subsubsection{Preparing The City Observation Set}

To train, test or otherwise run the CityNet model, we use a set of historical city observations $S=\left\{s^{0}, \ldots, s^{T}\right\}$, where $s^{t}=\left(q^{t}, \mu^{t}\right)$ for $t: 0 \rightarrow T$. The $q^{t}$ is the set of trip demand, and $\mu^{t}$ is the set of global attributes. Figure 4.7 illustrates the

\footnotetext{
${ }^{8}$ https://w2.weather.gov/climate/xmacis.php?wfo=okx
} 
flowchart for preparing city observations from trip records and weather information. We annotate the flowchart steps as follows:

- Step 1: Import all trip records and weather information within a fixed time window (in our case, for the year 2014).

- Step 2: Sort the data fields by time, then aggregate by date and subsequently by time interval (this results in 3285 aggregated groups of trip records per year).

- Step 3: For each group of trip records, build a list of trip requests. First overlay the trip pickup and dropoff coordinates on the census tracts. Then, conduct a point-in-polygon operation to find the pickup and dropoff nodes for each trip.

- Step 4: Build a list, $q^{t}$ where $t$ is the current time interval, by aggregating the trip requests for all node pairs. Then, collect global attributes, $\mu^{t}$, for the current time interval.

- Step 5: Finally, we store $\left(q^{t}, \mu^{t}\right)$ to memory and repeat the steps from step 3 until all data groups are processed into memory. 


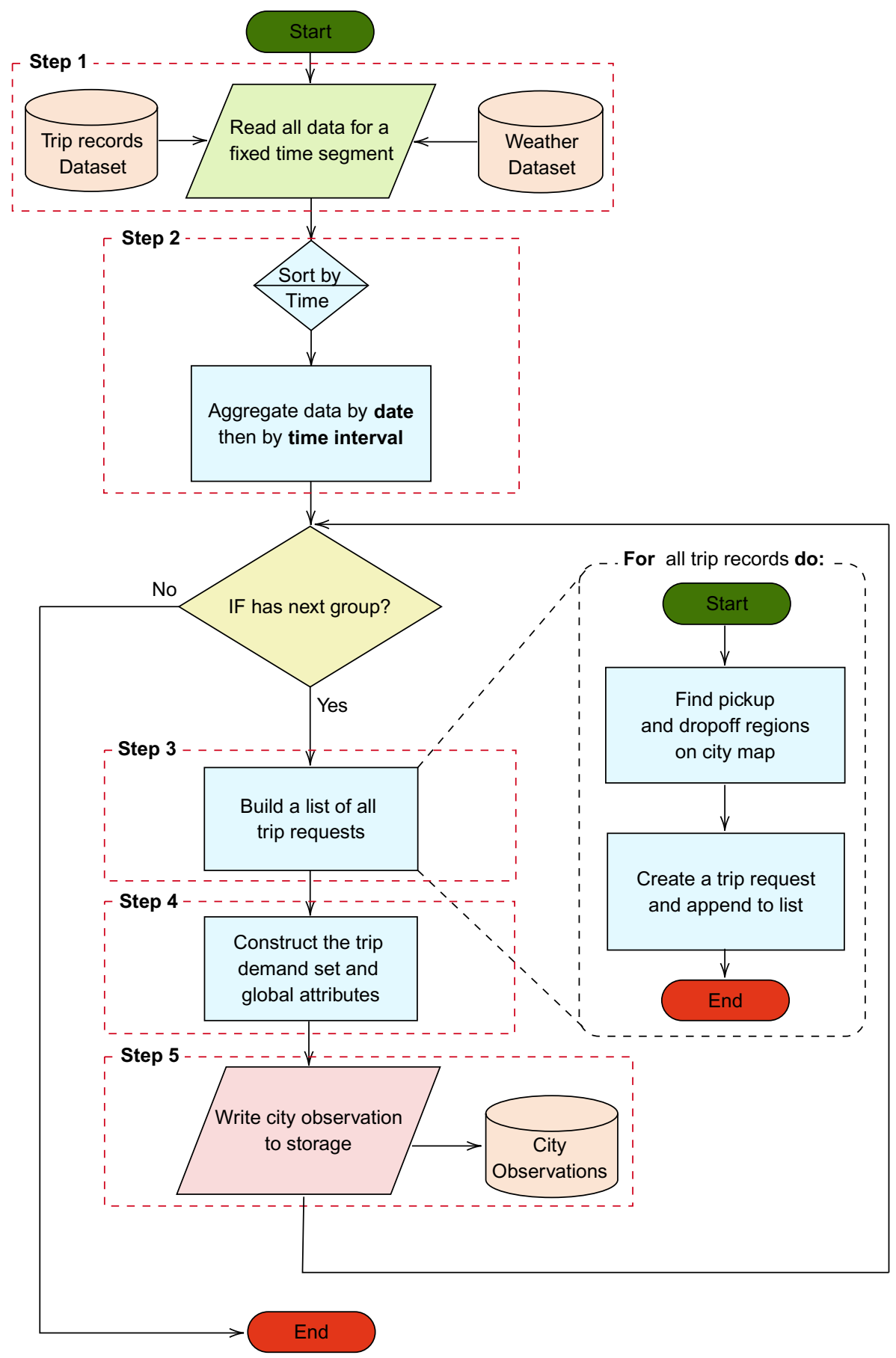

Figure 4.7: Preparing City Observations Flowchart 


\subsection{Data Analysis}

In this section, we analyze the effect of our selected socio-economic and land-use factors, pertaining to regions of NYC, on trip request volume. Note, the contribution of Residential-Medium, Residential-Small, and Commercial-Medium regions is relatively negligible in the charts. Therefore, for clarity, we group residential regions together and commercial regions together. All charts in this section were plotted per region per time interval.

Total Population Figure 4.8 shows that regions with a population of less than 500 exhibiting a high number of trip requests, which are predominantly commercial and airport regions. Additionally, the chart shows that trip requests for residential regions increase proportionally as population numbers increase. The same can be observed for mixed regions from the 1500 - 8999 range. However, trip volume varies for the commercial region, which indicates that they are not greatly affected by population numbers.

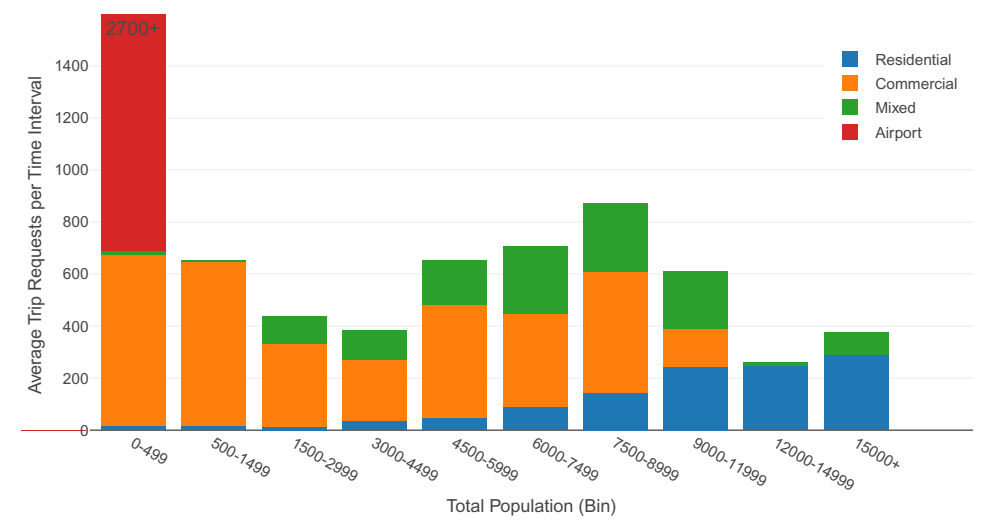

Figure 4.8: Correlation between total population per region and average trip requests per time interval, and marked by general land-use type. 
Median Income Figure 4.9 depicts the effect of household income on trip request levels. The increase in income indicates an exponential increase in trip requests, with the exception of zero income regions, which consists of commercial and airport regions. The bar chart shows a significant split in the number of trip requests between regions with an income greater than 100,000 and those with less, indicating that wealthier regions travel more.

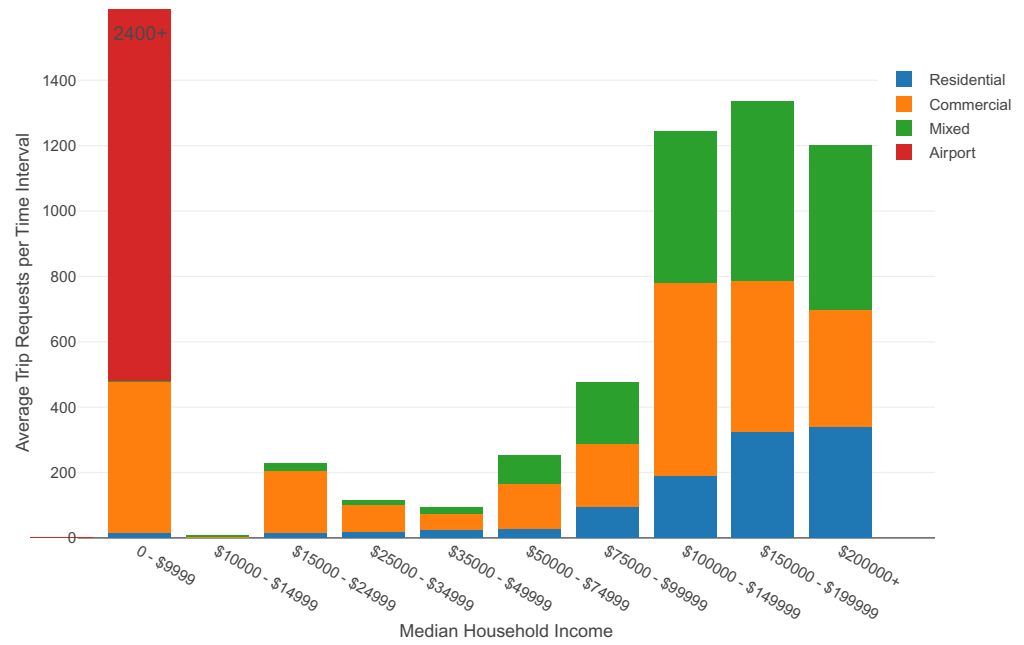

Figure 4.9: Median household income in relation to average number of trip requests per time interval, and marked by general land-use type.

Higher Education The correlation between higher education and the number of trips is evident in the data, as shown in Figure 4.10. The chart indicates that a welleducated population, $50 \%$ or higher, makes more trip requests than low educated ones, with the exception of the " $0-9^{\prime \prime}$ bin that has a large number of trips from airport regions. 


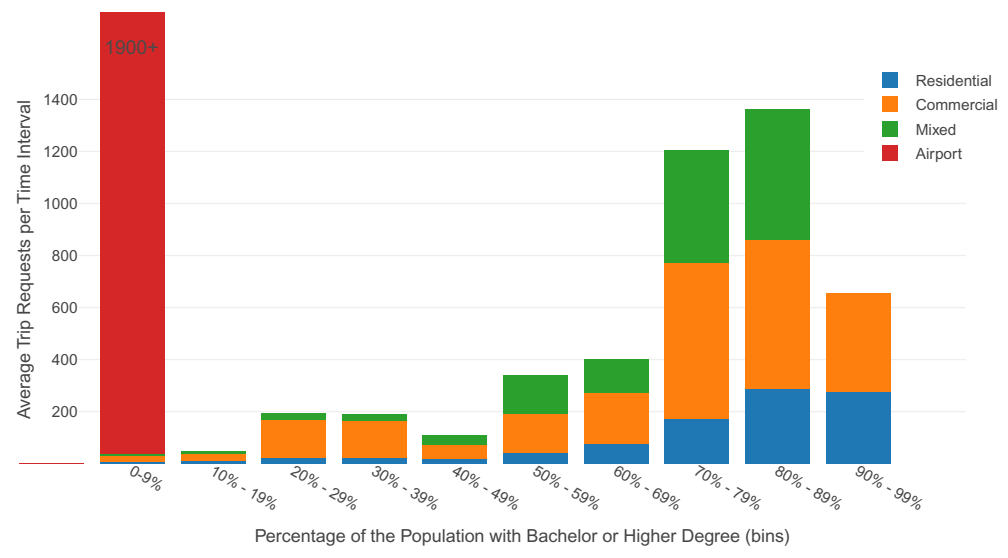

Figure 4.10: Average Trip count with respect to the $\%$ of population with bachelor or higher degree, and marked by general land-use type.

Land-use Figure 4.11 shows the number of trip requests per land-use type, which is dominated by large commercial and Airport regions. We note that the chart shows

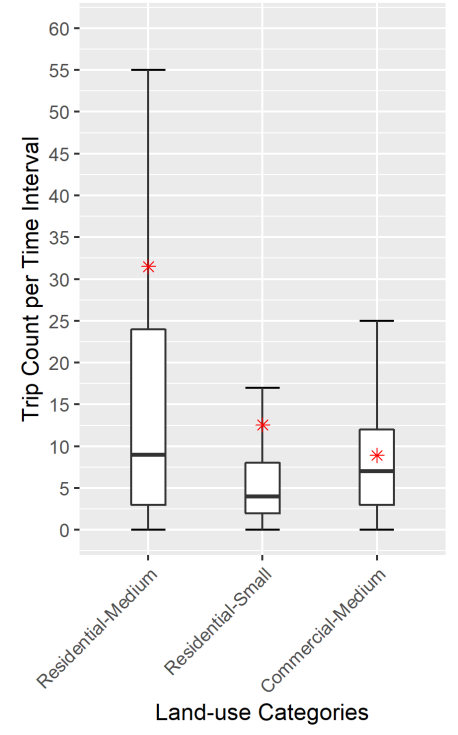

(a) Low Volume

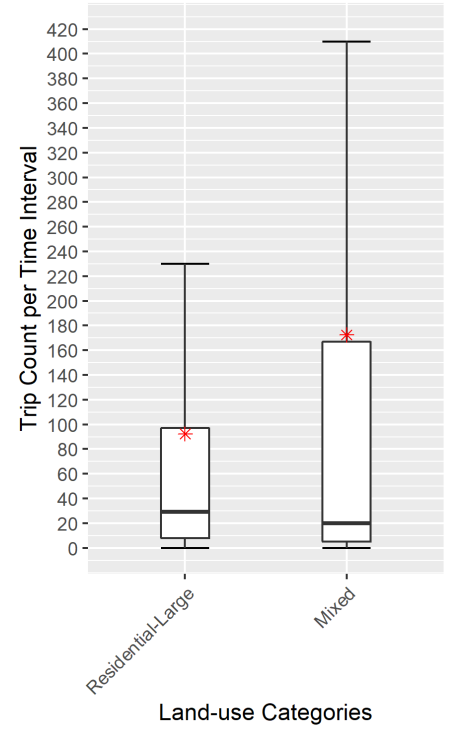

(b) Medium Volume

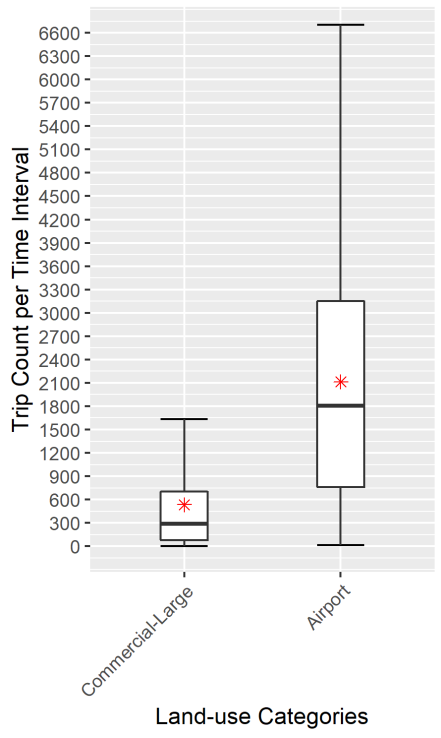

(c) High Volume

Figure 4.11: Box plot illustration of trip count by land-use type. The plots are grouped by trip volume level. The red star indicates the mean value. 
significant deviation for all region types. The low median compared to the average implies that there are many regions within each group that have low number of trip requests.

\subsection{Experiments}

For our experiments, we implemented all neural network based methods, including the ones used in our CityNet model, using the Tensorflow machine learning platform (developed by Martin Abadi et al. [69]), in conjunction with the Keras deep learning API (developed by François Chollet et al. [70]). Additionally, we used DeepMind's Graph-Net API [71] to implement the city graph structure used by our model. All experiments were executed and evaluated on a single Nvidia GeForce GTX 1070 GPU with $8 \mathrm{~GB}$ of memory.

In this section, we first describe the baseline methods, including two variants of our model, used to compare our proposed model with (Section 4.3.1). Then, we explain the data preprocessing steps undertaken before training the models (Section 4.3.2), followed by the metrics used to evaluate them (Section 4.3.3). We then detail the hyperparameters used to set up the machine learning models (Section 4.3.4). Lastly, we detail and discuss our experiment results (Section 4.3.5). 


\subsubsection{Comparison Methods}

We compare our CityNet model against four traditional statistical methods and three neural network methods found commonly used as baseline models in time-series forecasting research papers $[37,72,3,11,1,13,4]$. The four methods are the naive Historical Average (HA), Auto-Regressive Integrated Moving Average (ARIMA), Support Vector Regression (SVR), and Random Forest (RF). In addition, we compared our model with three neural network methods eXtreme Gradient Boosting [73] (XGBoost), Wide \& Deep neural network [74], and a graph neural network (GNN), which predicts edge only features (i.e, trip demand).

We further describe the settings and parameters used for the seven methods as follows:

- HA: The Historical Average predicts the trip demand for a given pair of regions based on the mean of previous trip demands that correspond the same time interval and day of the week.

- ARIMA: We used Löning et al. automated API [75] to discover the optimal parameters for the ARMIA model. We set the auto-regressive order to 12, differencing order to 1 , and the moving average order to 2 for best results.

- SVR: The objective of SVR is to minimize a coefficient to an acceptable error margin $\epsilon$. We use a Radial Basis Function kernel as the coefficient and an $\epsilon$ of 0.1 (API default).

- RF: Random forests is an ensemble estimator that uses decision trees built from sampling the training data. The output is the average prediction of the individual trees. 
- XGBoost: eXtreme Gradient Boosting is a tree boosting system that relies on decision tree ensembles to make prediction. The system implements machine learning algorithms to perform gradient boosting.

- WnD: This model relies on feature embedding, and jointly trains a linear neural network (logistic regression model) and a deep feed forward neural network. It is ideal for large-scale regression problems with numerous categorical features, such as recommender systems [74]. We construct the deep neural network with 5 hidden layers of size 256, 256, 1024, 512, and 128 neurons respectively.

- GNN: We implemented this model using DeepMind's Graph-Net [71] API. In this neural network we employed two Multi-Layered Perceptrons (MLP) to compute the updated node state and edge state respectively. The node MLP consists of two hidden layers of size 256 neurons each. The edge MLP consists of three hidden layers of size 1024, 512, and 128. The updated node states are used to compute the updated edge states, which in turn are regressed over to compute trip demand on the edges.

The number of neurons used for the WnD and GNN models coincides with the number of GRU units used for our node submodel, in addition to the number of neurons used for the FC layers of our edge submodel. We also compare with two variants of our model, as follows:

- CityNet-0: CityNet without incoming and outgoing trip prediction. More precisely, we pass the node attributes to the edge submodel without performing the node attribute update step. 
- CityNet-MLP: We replace the CNN in the edge submodel with an MLP that has three hidden layers 1024,512 , and 128 respectively (similar to the FC layers of the $\mathrm{CNN}$ ). We also flatten the edge feature matrix to a vector to comply with the allowed input-shape of the MLP. In addition, we use a 36 look-back window size.

For comparable experiments with our CityNet model, we use the same metadata for all baseline methods and variants, as well as the same loss function in equation 3.14 with the exception of RF (we used Mean Squared Error loss) due to API limitation.

\subsubsection{Preprocessing}

Authors of [76] and [77] suggest that input data to neural networks needs to be normalized to avoid biased parameter updates during the training process. To that effect, we standardize all input data to the models by removing the mean and scaling to unit variance, with the exception to XGBoost based on API recommendation. We also encode categorical data (e.g., land-use types) as a one-hot numerical array, where each category is converted to a unique binary variable.

Further, to ensure a close to uniform distribution of trip request values during the training process, we classify the edges by their respective number of trip requests and then group by time interval. In our case we have 350 cluster of edges and 3285 time intervals for a total of 1,149,750 classes. Each class consists of all the edges with the same number of trips for a unique time interval in the year. We then randomly select an $x$ number of records (or less) from each class, where $x$ is a predefined value. In our experiments we select 200 records from each class. From that, we split the data into 
training, validation, and testing sets using a $60 \%-20 \%-20 \%$ split, respectively. We use the training set to train the models, the validation set to ensure that the models are not over-fitting, and to early-stop the neural network training process. The testing set is used to evaluate the models' performance. Moreover, for the CityNet models and variants, specifically, we split the data as follows:

- Node Submodel: We split the graph nodes by land-use type into training and testing nodes, as specified in Table 4.3. This allows us to test the node submodel's ability to generalize its localized predictions to new nodes.

- Edge Submodel: We reintroduce the test nodes and their connections into the graph and carry out the same data split mentioned previously.

Table 4.3: Train and test split by land-use category

\begin{tabular}{|r|cc|}
\hline Land-Use & Training & Testing \\
\hline \hline Residential-Large & 130 & 32 \\
\hline Residential-Medium & 224 & 56 \\
\hline Residential-Small & 44 & 11 \\
\hline Commercial-Large & 66 & 17 \\
\hline Commercial-Medium & 14 & 3 \\
\hline Mixed & 146 & 37 \\
\hline Airport & 1 & 1 \\
\hline Total & $\mathbf{6 2 5}$ & $\mathbf{1 5 7}$ \\
\hline
\end{tabular}

\subsubsection{Evaluation Metrics}

We evaluate the baseline models, including our CityNet model and its variants using Mean Squared Logarithmic Error (MSLE), Root Mean Squared Error (RMSE), and Mean Magnitude of Relative Error (MMRE), defined as follows: 


$$
\begin{gathered}
M S L E=\frac{1}{n} \sum_{i=1}^{n}\left(\log _{e}\left(\hat{y}_{i}+1\right)-\log _{e}\left(y_{i}+1\right)\right)^{2}, \\
R M S E=\sqrt{\frac{1}{n} \sum_{i=1}^{n}\left(\hat{y}_{i}-y_{i}\right)^{2}} \\
M M R E=\frac{1}{n} \sum_{i=1}^{n}\left|1-\frac{\hat{y}_{i}}{y_{i}}\right|
\end{gathered}
$$

where $n$ is the number of samples, $\hat{y}_{i}$ is the inferred value of the $i^{\text {th }}$ sample and $y_{i}$ is the corresponding actual value. We chose MSLE as the main metric of comparison, since it is part of the loss functions in equations 3.14, while RMSE was chosen to measure the distance of the inferred values from the fitted line. Additionally, we found that MMRE is a good measure of model accuracy even though it favors models that underestimate.

\subsubsection{Hyperparameters}

For the machine learning models, we initialize the learning rate, $\eta$, to 0.01 in conjunction with a step decay function that decreases $\eta$ with a decay factor of $\frac{3}{4}$ every five epochs (determined empirically). Gradually reducing the learning rate helps the model generalize better by escaping local minima $[78,79]$. We also set the SGD momentum value to 0.9 , as suggested by [80]. We train the models over 100 epochs (1000 for XGBoost), and use an early-stopping function, following the recommenda-

tions of $[81,82,83]$, on the validation loss to prevent over-fitting the models. To avoid over-fitting further, we use an L2-norm for weight regularization with a value of 0.001 $\left(1 X 10^{-4}\right.$ for $\left.\mathrm{WnD}\right)$ on all trainable layers. 
Furthermore, the CityNet model has an extra hyperparameter, the look-back window. In our experiments, we compared two forms of look-back window: sequential windows defined as $n$ consecutive time intervals, denoted by $\omega$ (default), and crosssection windows defined as $n$ time intervals with a gap of delta between the intervals, denoted by $\omega c$. In our experiments we use a delta of one week for the same time interval (for example 9 past Monday-Mornings). Since there are 52 weeks in our training data, we compute the cross-section by shifting the days, where the day that rolls beyond the last position is re-introduced at the first, making the assumption that trip request values for the past year are similar to the current year. We set $\omega$ to $9,18,27$, and 36 window size (multiples of the number of time intervals in a day) and discuss the results in the following section. We note that 36 is the computational limit for our system setup given the number of data features and batch size.

\subsubsection{Experimental Results}

In table 4.4, we tabulate the performance results for the baseline methods, CityNet variants, and our proposed CityNet model with different $\omega$ sizes and forms. In addition, we use the bar charts in Figure 4.12 for a clear comparison between the evaluation results. Note, in Section 4.3.5.1 and 4.3.5.2, we only discuss the metrics for trip demand prediction, and leave incoming/outgoing trip prediction discussion for Section 4.3.5.5 as they are not computed using the baseline methods. 
Table 4.4: Experimental Results

\begin{tabular}{|l|lll|}
\hline Model & MSLE & RMSE & MMRE \\
\hline \hline Baseline & & & \\
HA & 2.8846 & 7.8769 & 0.7978 \\
ARIMA & 0.6148 & 5.3603 & 0.8855 \\
SVR & 0.2184 & 5.1890 & 0.4422 \\
RF & 0.1642 & 4.8353 & 0.3831 \\
XGBoost & 0.1612 & 4.9200 & 0.3521 \\
WnD & 0.1346 & 5.2169 & 0.3157 \\
GNN & 0.1420 & 3.3700 & 0.2667 \\
\hline Variants & & & \\
CityNet-MLP & 0.1176 & 4.5400 & 0.2877 \\
CityNet-0 & 0.1015 & 4.1713 & 0.2710 \\
\hline CityNet & & & \\
$\omega 9$ & 0.0965 & 3.7949 & 0.2618 \\
$\omega 18$ & 0.0881 & 3.5594 & 0.2496 \\
$\omega 27$ & 0.0867 & 3.5404 & 0.2442 \\
$\omega 36$ & $\mathbf{0 . 0 8 6 2}$ & 3.5387 & $\mathbf{0 . 2 4 3 2}$ \\
$\omega c 18$ & 0.0900 & 3.3183 & 0.2477 \\
$\omega c 27$ & 0.0873 & $\mathbf{3 . 1 9 7 8}$ & 0.2458 \\
$\omega c 36$ & 0.0865 & 3.2241 & 0.2440 \\
\hline
\end{tabular}

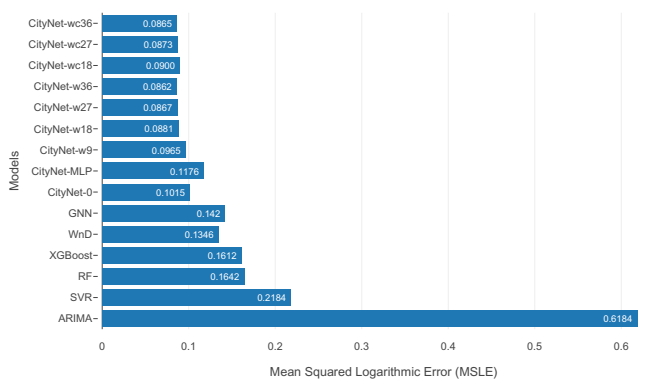

(a) MSLE Results

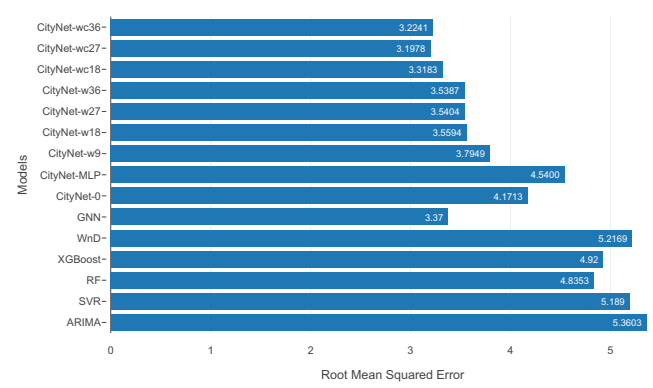

(b) RMSE Results

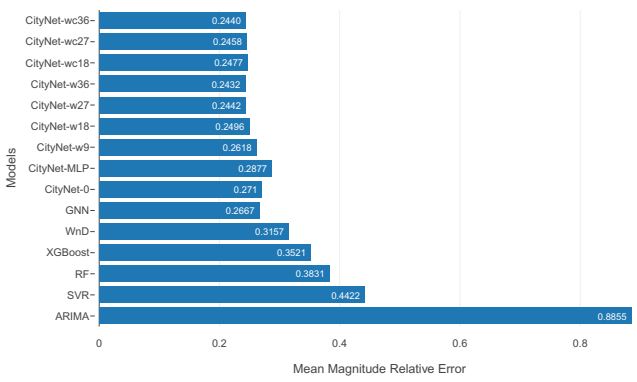

(c) MMRE Results

Figure 4.12: Bar charts illustrating the evaluation results. 


\subsubsection{Performance Comparison with Baseline Methods}

Figure 4.12a and 4.12c shows that our proposed CityNet model (we let CityNet- $\omega 36$ be the compared model) outperforms all the baseline methods and the variants on the MSLE and MMRE metrics but exhibits comparable results on the RMSE metric displayed in Figure 4.12b. More specifically, HA and ARIMA, which rely solely on historical observations to generate predictions, perform the worst across all metrics. Evaluation results gradually become better with SVR, which considers contextual data features for demand prediction, but achieves a 153\% higher MSLE value than our model. Decision tree methods, RF and XGBoost, perform slightly better with proportional MSLE values at 0.1642 and 0.1612 , respectively, which is $87 \%$ higher than our model.

Moreover, neural network methods perform significantly better since they are capable of modeling complex non-linear relationships. WnD achieves the lowest MSLE value (0.1346) amongst the baseline methods (56\% higher than CityNet- $\omega 36)$ but achieves a relatively high RMSE (5.2169). GNN, on the other hand, produces a $5 \%$ higher MSLE (0.142) than WnD but 54\% lower RMSE (3.37), which is a high margin and better than our selected model by $5 \%$. This indicates that the GNN model underestimates for large trip values while maintaining adequate estimation for low trip values. Additionally, in Figure 4.13 we illustrate the trip prediction results for four separate regions with their respective actual trip values for the XGBoost, $\mathrm{WnD}$, GNN and CityNet- $\omega 36$ models. We observer that the GNN model's forecasts are lower than the fitted line for all illustrated regions as indicated previously. The $\mathrm{WnD}$ and XGBoost models conform better for the Airport and Commercial-Large regions 
but show lower forecast results for the Mixed region, while showing higher forecasts for the Residential-Large region. The CityNet- $\omega 36$ model, on the other hand, shows similar performance to $\mathrm{WnD}$ and XGBoost for the Airport, Commercial-Large, and Residential-Large regions but a better fit on the Mixed region.

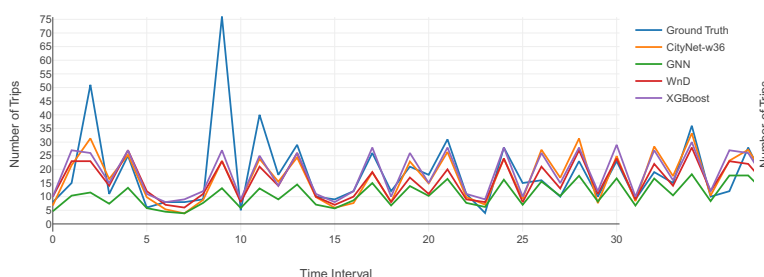

(a) Airport Region Predictions

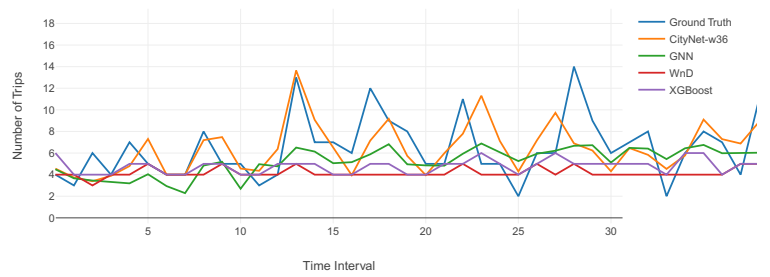

(c) Mixed Region Predictions

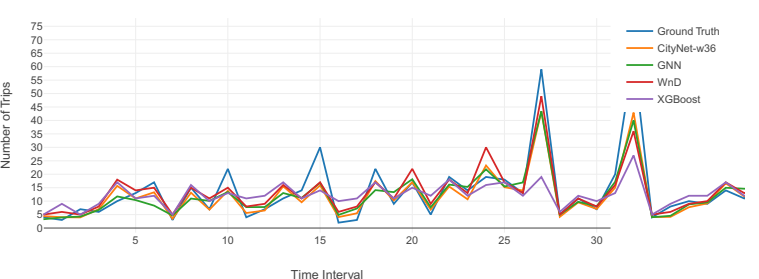

(b) Commercial-Large Region Predictions

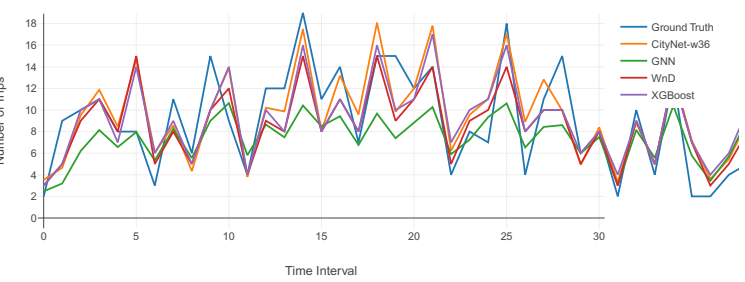

(d) Residential-Large Predictions

Figure 4.13: Comparison between the actual trip demand values for four separate regions (labeled by land-use type) and their respective predicted values for XGBoost, WnD, GNN and CityNet- $\omega 36$ models

Further, in Figure 4.14 ( $\mathrm{a}$ and b), we show the performance of XGBoost, WnD, GNN, and CityNet- $\omega 36$ models on different time intervals and days of the week, respectively. We observe that the CityNet- $\omega 36$ consistently outperforms the remaining models and can maintain its performance across all time intervals $(0.087 \pm 0.003)$ and between weekdays and weekends $(0.087 \pm 0.001)$. These results indicate that our 
model is robust for all days of the week and time intervals.

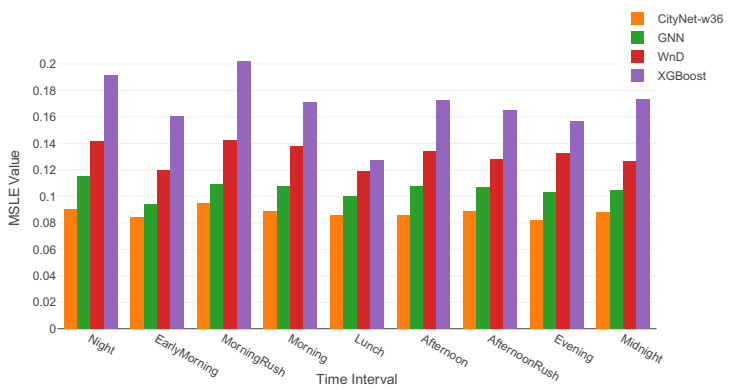

(a) MSLE Values by Time Interval

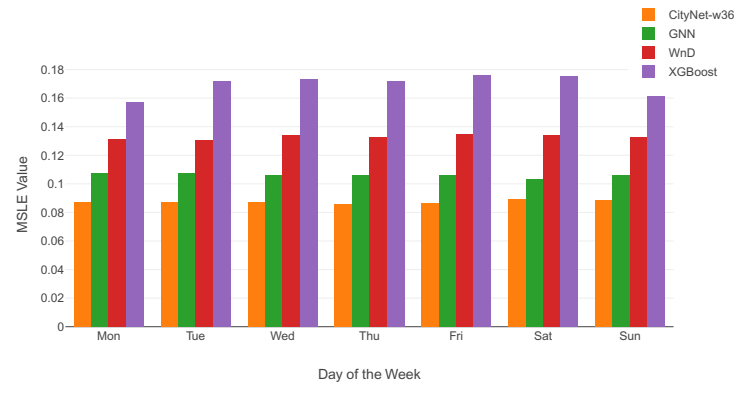

(b) MSLE Values by Day of the Week

Figure 4.14: Comparison between MSLE values for XGBoost, WnD, GNN and CityNet- $\omega 36$ models.

\subsubsection{Performance Comparison with CityNet-Variants}

The variants of our model, CityNet-MLP and CityNet-0, reached lower MSLE values (0.1176 and 0.1015 , respectively) than the baseline models. Compared to $\mathrm{WnD}$, they produce $14 \%$ and $32 \%$ lower MSLE values, respectively. When compared to GNN, they resulted in higher RMSE ( $34 \%$ and $23 \%$, respectively) and marginally higher MMRE ( $8 \%$ and 1\%, respectively). Additionally, both variants, compared to CityNetw36, obtained higher error values for all metrics. Specifically, CityNet-0 scored 17\% higher MSLE and RMSE values, which implies that trip demand prediction is affected by the incoming and outgoing trip values of the origin and destination nodes. This observation is intuitive since incoming and outgoing trip values are an aggregation of trip requests. Moreover, replacing the CNN with an MLP, as with the CityNet-MLP variant, resulted in lower performance than CityNet-0 (15\% higher MSLE and 8\% higher RMSE), which is unexpected since the incoming and outgoing trip values were 
part of the feature vector. This observation highlighted the importance of the CNN in capturing an implicit relationship between the pair-wise node attributes of an edge.

In addition, we compare the CityNet-Variants' trip demand forecasting results for the same four regions as the previous section. We observer that the prediction results for CityNet- $\omega 36$, CityNet-MLP, and CityNet-0 are close for the Airport, CommercialLarge, and Residential-Large regions, depicted in 4.15a, 4.15b, and 4.15d respectively. However, for the Mixed region (Figure 4.15c), CityNet-MLP produces low forecast results, CityNet-0 produces slightly larger trip numbers, while CityNet- $\omega 36$ achieves relatively the best fit values to the ground truth. We speculate that the difference in

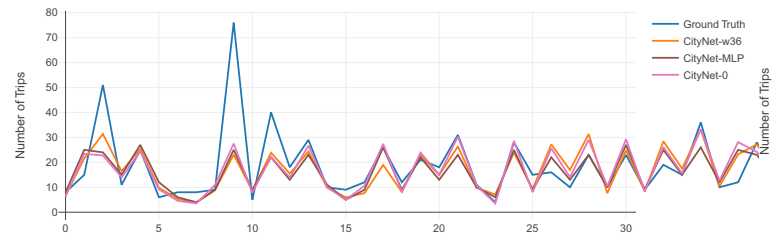

(a) Airport Region Predictions

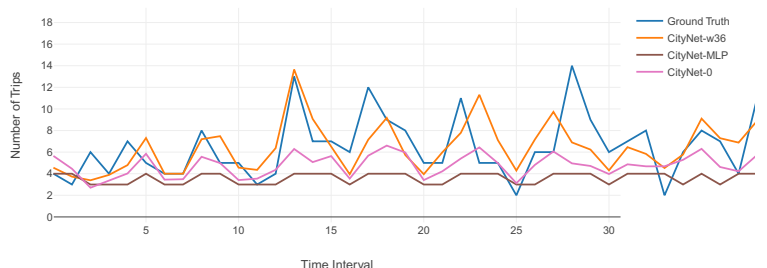

(c) Mixed Region Predictions

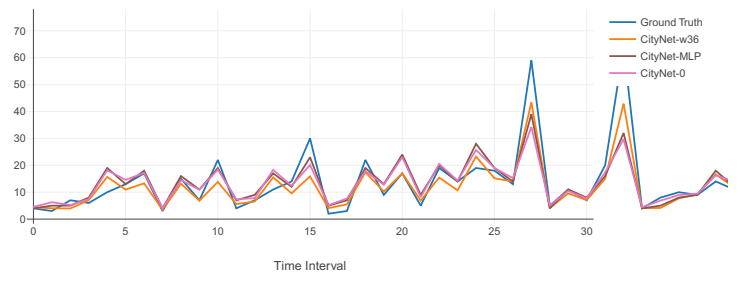

(b) Commercial-Large Region Predictions

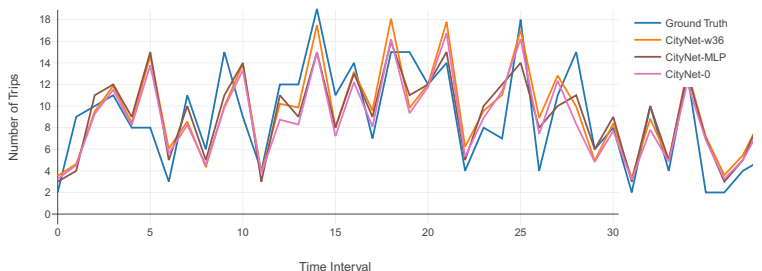

(d) Residential-Large Predictions

Figure 4.15: Comparison between the actual trip demand values for four separate regions (labeled by land-use type) and their respective predicted values for CityNet0 , CityNet-MLP and CityNet- $\omega 36$ models 
model performance between the Mixed region and the Airport, Commercial-Large, and Residential-Large regions is due to the Mixed regions' trip data exhibiting a low median (18) and a high standard deviation (325) compared to the other regions.

Furthermore, in Figure 4.16 ( $\mathrm{a}$ and $\mathrm{b}$ ), we show the performance of CityNet-0, CityNet-MLP, and CityNet- $\omega 36$ models on different time intervals and days of the week, respectively. Similar to the results in Section 4.3.5.1, we observe that the CityNet- $\omega 36$ consistently outperforms both variant methods and can maintain its performance across all time intervals and days of the week. We also observe that CityNet-0 produces stable results across all time intervals $(0.099 \pm 0.004)$ and days of the week $(0.099 \pm 0.001)$, which further confirms the importance of the CNN in producing robust results.

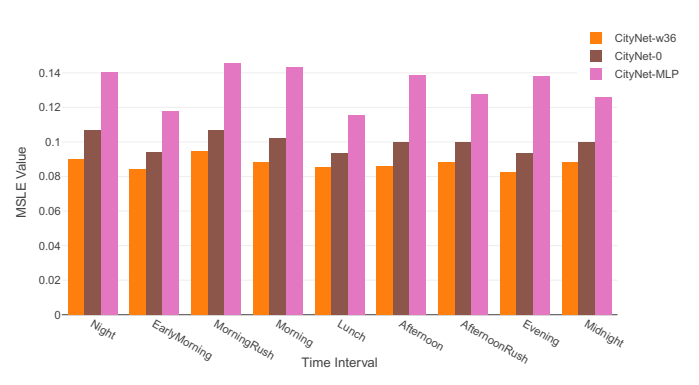

(a) MSLE Values by Time Interval

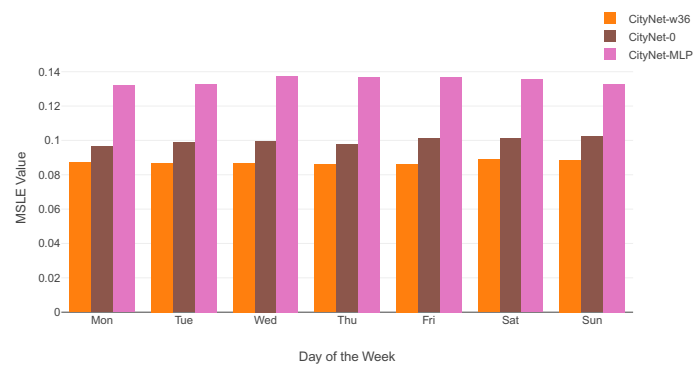

(b) MSLE Values by Day of the Week

Figure 4.16: Comparison between MSLE values for CityNet-0, CityNet-MLP, and CityNet- $\omega 36$ models.

\subsubsection{Performance Comparison with Alternative (Untested) Models}

The ST-ResNet[1], STGCN[4], DCRNN[14], DMVST-Net[3], ST-MGCN[13], and STED-RMGC[84] are all state-of-the-art models that were developed to address similar 
tasks to trip demand prediction. Due to their implementation complexity, we were unable to implement them for direct comparison with our CityNet model. However, we compare the evaluation results mentioned in their respective papers to that of our model. In Table 4.5 we tabulate the provided metric evaluation results for each model, as well as the data source used to train it. From the table, we can see Table 4.5: Evaluation results for the alternative models as found on their respective papers.

\begin{tabular}{|l|lll|}
\hline Model & RMSE & MMRE & Data Source \\
\hline \hline ST-ResNet & 16.69 & N/A & Taxi data for Beijing, China \\
STGCN & 5.20 & 0.0911 & Beijing Municipal Traffic Commission \\
DCRNN & 5.38 & 0.073 & Los Angeles County traffic data \\
DMVST-Net & 9.642 & 0.1616 & Taxi data from Didi Chuxing, China \\
ST-MGCN & 10.78 & 0.088 & Ride-hailing datasets for Beijing, China \\
ST-ED-RMGC & 4.29 & 0.380 & NYC-TLC for-hire-vehicle records \\
CityNet- $\omega 36$ & 3.538 & 0.2432 & NYC-TLC green and yellow taxi records \\
\hline
\end{tabular}

that our CityNet model achieves the lowest RMSE value (3.538) amongst all the models but a 50\%-176\% higher MMRE value than STGCN, DCRNN, DMVST-Net, and ST-MGCN models. Notably, when analyzing our own models' performance, we observed that the prediction results of a model adhere to the mean of the sampled data when they achieve a high RMSE value but distinguishably low MMRE value. This outcome is not desirable since the mean of a strongly positive skewed dataset is a relatively small value. In other words, since the MMRE metric is biased to models that underestimate (it applies a higher penalty when actual < predicted value), as with the case of STGCN, DCRNN, DMVST-Net, ST-MGCN, and the GNN baseline model. However, if both the RMSE and MMRE results were linearly correlated, as with the ST-ED-RMGC and the CityNet models, the estimations would approximate 
more closely the trend of the fitted line.

\subsubsection{Effect of Different Components on Model Performance}

At the start of our work, we experimented with various model components and hyperparameters, which resulted in the current CityNet model setup as depicted in Figure 3.4 and 3.7. Table 4.6 shows the setup for some of our initial models with the biggest improvements. We train the initial models with a look-back window size of Table 4.6: Experimental model configurations. The convolution operation (conv) takes 2 parameters, kernel size, denoted by $\mathrm{k}$, and a stride dimension, denoted by $\mathrm{s}$.

\begin{tabular}{|c|c|c|c|c|}
\hline & CityNet-01 & CityNet-02 & CityNet-03 & Current Model \\
\hline $\begin{array}{l}\text { Node } \\
\text { sub- } \\
\text { model }\end{array}$ & $\begin{array}{l}\text { GRU(64)-GRU(64)- } \\
\text { GRU(64)-GRU(64) }\end{array}$ & GRU(256)-GRU(256) & $\begin{array}{l}\text { GRU(256)-GRU(256)- } \\
\text { GRU(512) }\end{array}$ & GRU(256)-GRU(256) \\
\hline $\begin{array}{l}\text { Edge } \\
\text { sub- } \\
\text { model }\end{array}$ & $\begin{array}{l}\operatorname{conv}(\mathrm{k}=512, \mathrm{~s}=(1 \times 3)) \\
\operatorname{conv}(\mathrm{k}=512, \mathrm{~s}=(2 \times 1))\end{array}$ & $\begin{array}{l}\operatorname{conv}(\mathrm{k}=32, \mathrm{~s}=(1 \times 3)) \\
\operatorname{conv}(\mathrm{k}=64, \mathrm{~s}=(1 \times 3)) \\
\operatorname{conv}(\mathrm{k}=128, \mathrm{~s}=(2 \times 1))\end{array}$ & $\begin{array}{l}\operatorname{conv}(\mathrm{k}=32, \mathrm{~s}=(1 \times 3)) \\
\operatorname{conv}(\mathrm{k}=64, \mathrm{~s}=(1 \times 3)) \\
\operatorname{conv}(\mathrm{k}=128, \mathrm{~s}=(1 \times 3)) \\
\operatorname{AvgPooling}(\mathrm{s}=(2 \times 1)) \\
\operatorname{conv}(\mathrm{k}=256, \mathrm{~s}=(2 \times 1)) \\
\operatorname{conv}(\mathrm{k}=512, \mathrm{~s}=(1 \times 3))\end{array}$ & $\begin{array}{l}\operatorname{conv}(\mathrm{k}=32, \mathrm{~s}=(1 \times 3)) \\
+ \text { Batch_Norm } \\
\operatorname{conv}(\mathrm{k}=64, \mathrm{~s}=(1 \times 3)) \\
+ \text { Batch_Norm } \\
\operatorname{conv}(\mathrm{k}=128, \mathrm{~s}=(1 \times 3)) \\
+ \text { Batch_Norm } \\
\operatorname{conv}(\mathrm{k}=256, \mathrm{~s}=(2 \times 1)) \\
+ \text { Batch_Norm } \\
\operatorname{conv}(\mathrm{k}=512, \mathrm{~s}=(2 \times 1)) \\
+ \text { Batch_Norm } \\
\text { AvgPooling }(\mathrm{s}=(2 \times 1))\end{array}$ \\
\hline & \multicolumn{4}{|c|}{ Flatten } \\
\hline & \multicolumn{4}{|c|}{$\begin{array}{c}\mathrm{FC}(1024)+\operatorname{Dropout}(0.4) \\
\mathrm{FC}(512)+\operatorname{Dropout}(0.4) \\
\mathrm{FC}(128)+\operatorname{Dropout}(0.2)\end{array}$} \\
\hline
\end{tabular}

18, and thus compare it with CityNet- $\omega 18$. We show the evaluation results in Table 4.7. We observe that models with more GRU layers (CityNet-01 and CityNet-03) did not greatly improve the results from their predecessors. In addition, increasing the kernel size for the convolution layers also did not provide significant improvement. The greatest gain was attained from decreasing the model complexity and adding 
Table 4.7: Evaluation results for the different model configurations.

\begin{tabular}{|l|lll|}
\hline Model & MSLE & RMSE & MMRE \\
\hline \hline CityNet_01 & 0.1022 & 4.0011 & 0.3404 \\
CityNet_02 & 0.0960 & 4.1180 & 0.2780 \\
CityNet_03 & 0.0935 & 4.0972 & 0.2754 \\
CityNet (our model) & $\mathbf{0 . 0 8 8 1}$ & $\mathbf{3 . 5 5 9 4}$ & $\mathbf{0 . 2 4 3 2}$ \\
\hline
\end{tabular}

the average-pooling layer, as seen for CityNet-03 and our current model. Lastly, the highest decrease in error rate was achieved when we added batch normalization layers and moved the average-pooling to the end of the convolution network.

\subsubsection{Effect of $\omega$ Size on the Node Submodel's Performance}

The number of historical trip requests (i.e., the look-back window size, $\omega$ ) used in estimating future trip demand has an effect on the node submodel's performance, to an extent. The chart in Figure 4.17 shows the validation results of the node submodel during training, where one day, i.e., 9 consecutive time intervals, has the highest error value at 2.76 , which is $22 \%$ - $31 \%$ higher compared to larger sequential window sizes. The chart indicates that $\omega 18$ - $\omega 36$ exhibit a close error rate, with $\omega 27$ holding the lowest value at 2.10. Further, using cross-section windows, $\omega c$, gives expectedly lower error values with a higher number of intervals, indicating strong long-range temporal dependencies. The lowest attained error loss value (1.68) is achieved with an $\omega c 36$. However, we note that $\omega c 36$ exhibits large error fluctuations before it stabilizes around the $60^{\text {th }}$ epoch, which indicates that a $\omega c 27$ performs better with less epochs. 


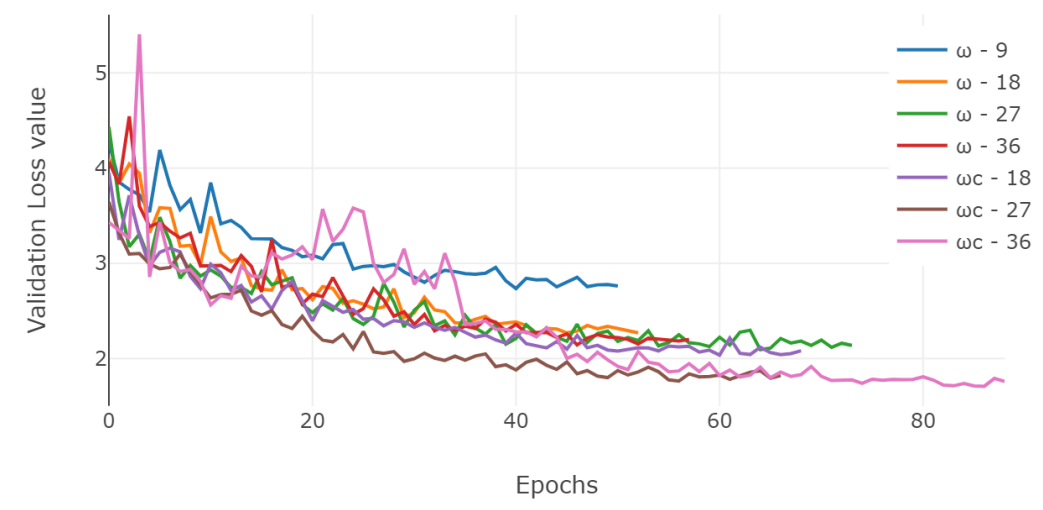

Figure 4.17: Node submodel validation results during training with respect to varying sequential and cross-section $\omega$ values.

We further demonstrate the MSLE results of the node submodel in Figure 4.18a with respect to varying, sequential, and cross-section window sizes. We see that the node submodel shows significant gains as we increase the window size, especially when using the cross-section method. An $\omega 36$ displays a $42 \%$ improvement over an $\omega 9$, while an $\omega c 36$ exhibits a $23 \%$ improvement over an $\omega c 18$. However, both forms converge around $0.16 \pm 0.003$ error value. Further, Figure $4.18 \mathrm{~b}$ shows minor gains on the RMSE metric. An $\omega 36$ displays a $7 \%$ improvement over an $\omega 9$, while an $\omega c 27$ holds the lowest error value, which is $17 \%$ lower than $\omega c 18$ and $6 \%$ lower than $\omega c 36$. Notably, the RMSE values from Figure 4.18b are substantially high. Due to RMSE being a measure of the standard deviation of the residuals, we affiliate these high numbers to the large standard deviation in trip request values per region land-use type (illustrated in Figure 4.11). We further discuss our results per region land-use type in Sections 4.3.5.7 and 4.3.5.8. 


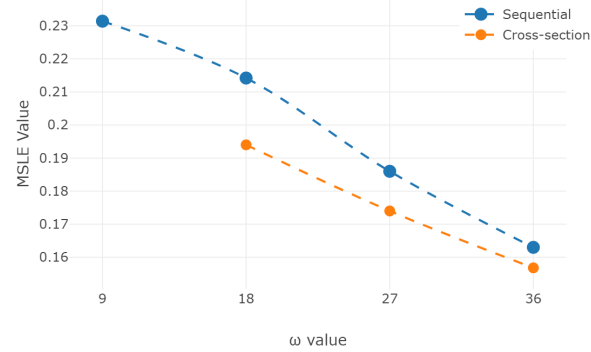

(a) MSLE Results

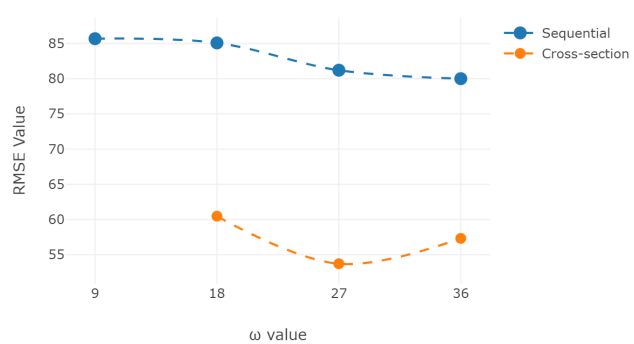

(b) RMSE Results

Figure 4.18: Evaluation metric results for varying sequential and cross-section $\omega$ values.

\subsubsection{Effect of $\omega$ Size on the Edge Submodel's Performance}

Viewing the edge submodel's validation results in Figure 4.19, we find a trend is maintained with varying sequential and cross-section window sizes, similar to that of the node submodel. The edge submodel exhibits a constant gap $(0.03 \pm 0.003)$ between both forms of the look-back window. We further observe that $\omega 18-\omega 36$ converge to the same error rate $(0.343 \pm 0.002)$ and outperform $\omega 9$ by $5 \%$.

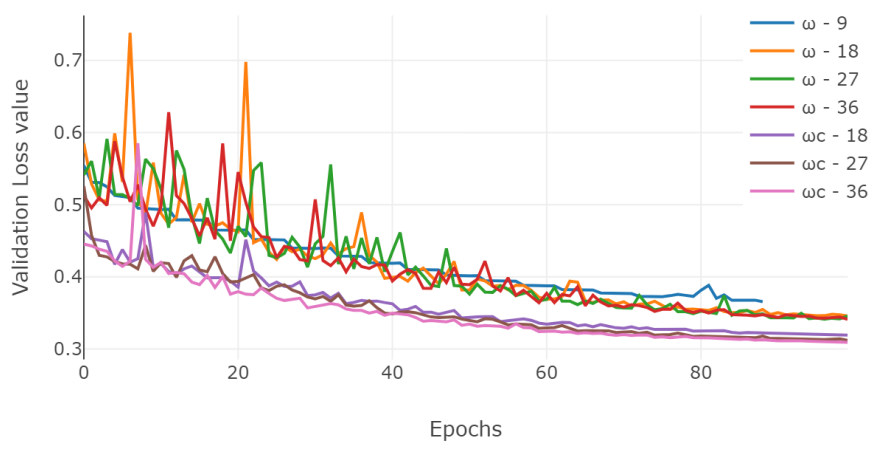

Figure 4.19: Edge submodel validation results during training with respect to varying lengthwise and cross-section $\omega$ values. 
Additionally, as expected, the loss values for all cross-section windows are lower than that of the sequential windows by a minimum of $9 \%$. It is also clear from the chart that $\omega c 18-\omega c 36$, similar to the sequential windows, converge to a close point $(0.313 \pm 0.006)$. This suggests that the edge submodel is not significantly effected by the accuracy of incoming and outgoing trip request predictions, but rather a close approximation.

This observation is further confirmed in Figure 4.20 where the MSLE and RMSE saturates beyond 18 time intervals for sequential windows, and does not improve for increased cross-section window sizes.

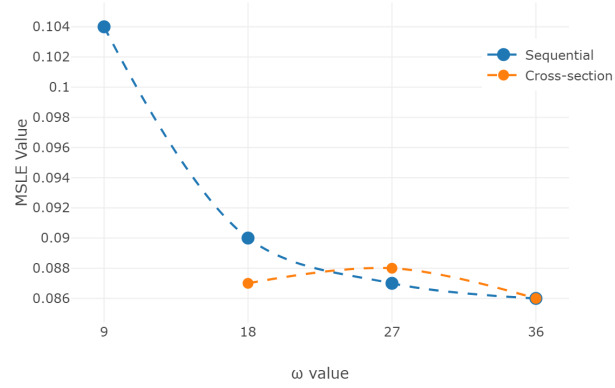

(a) MSLE Results

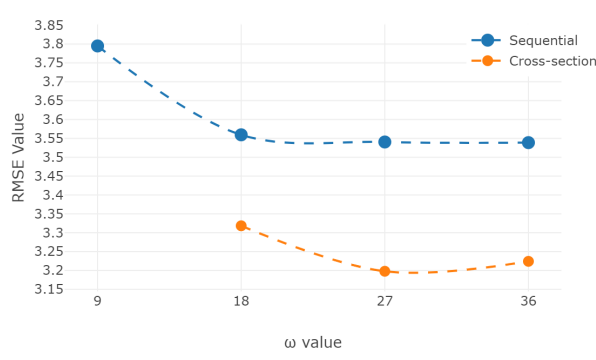

(b) RMSE Results

Figure 4.20: Evaluation results for varying sequential and cross-section $\omega$ values.

\subsubsection{Node Submodel's Performance Results per Land-Use Category}

Here we discuss the outgoing trip request prediction results by region land-use category on the CityNet- $\omega 36$ model. We note that the incoming trip requests are closely correlated to the outgoing and, therefore, will not be analyzed. In Figure 4.21 we observer that the node submodel is able to predict a close approximation to the global average and standard deviation for each category, except Airport regions. More 


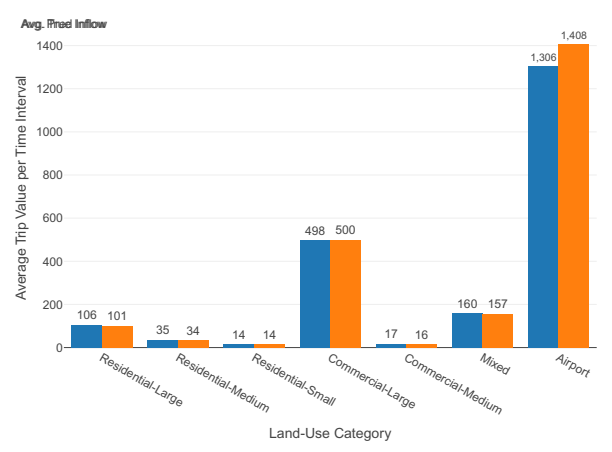

(a) Average

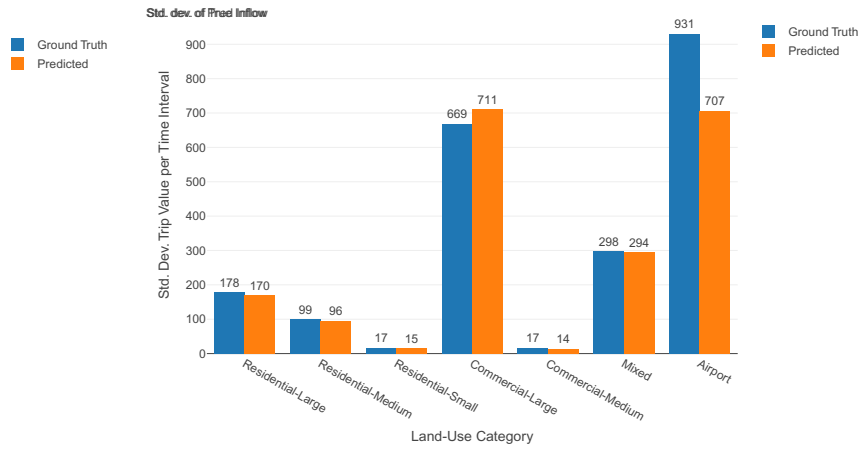

(b) Standard deviation

Figure 4.21: The average and standard deviation of outgoing trip requests by region land-use category.

specifically, in Figure 4.22 ( $\mathrm{a}$ and b) we see that the model's forecasting error rate is different for each land-use category. The Airport regions have the highest error rate for both MSLE and RMSE, 0.672 and 649.1 respectively. We see from Figure 4.23

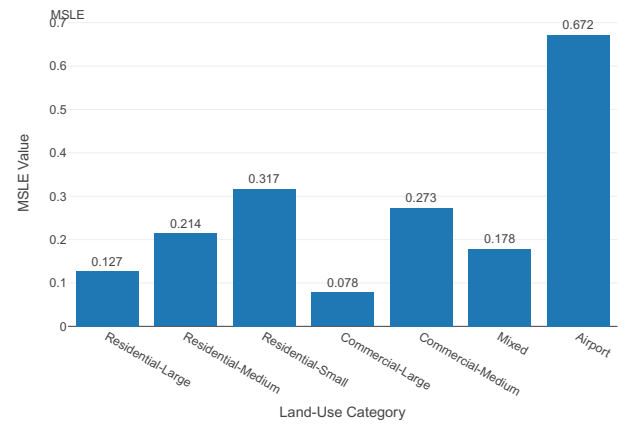

(a) MSLE results

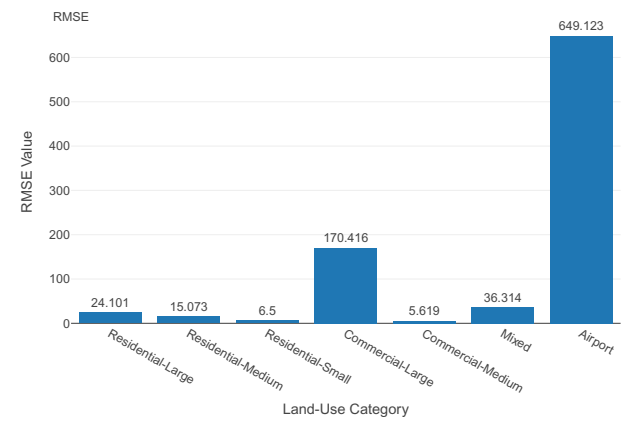

(b) RMSE results

Figure 4.22: Evaluation results per region land-use category.

that the outgoing predictions of our model provide a good fit to the ground truth. However, the model over-estimates the predictions of many relatively low trip volume intervals (in the range of $[30,170]$ outgoing trip requests), causing MSLE values > 3.3 and RMSE values > 464.3 on average. 


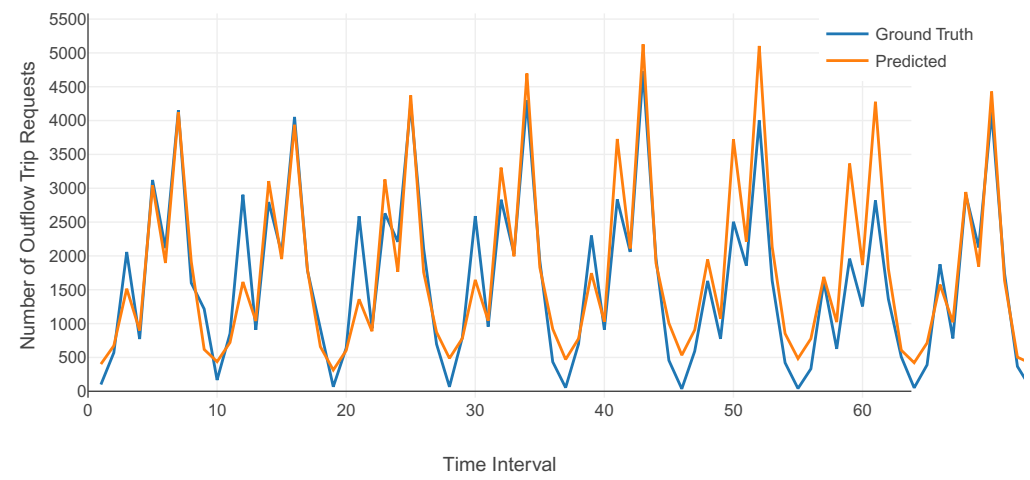

Figure 4.23: Comparison between the predicted outgoing trip requests and it respective actual values for an Airport region.

Moreover, the model achieves the lowest MSLE value (0.078) for the CommercialLarge regions but a relatively high RMSE value (170.4). This indicates that there are specific regions within the category that cause error spikes. We highlight this observation in Figure 4.24 where we observe that the region "1225076148348", which is a central train station in the city, is resulting in a relatively high RMSE value (545.3).

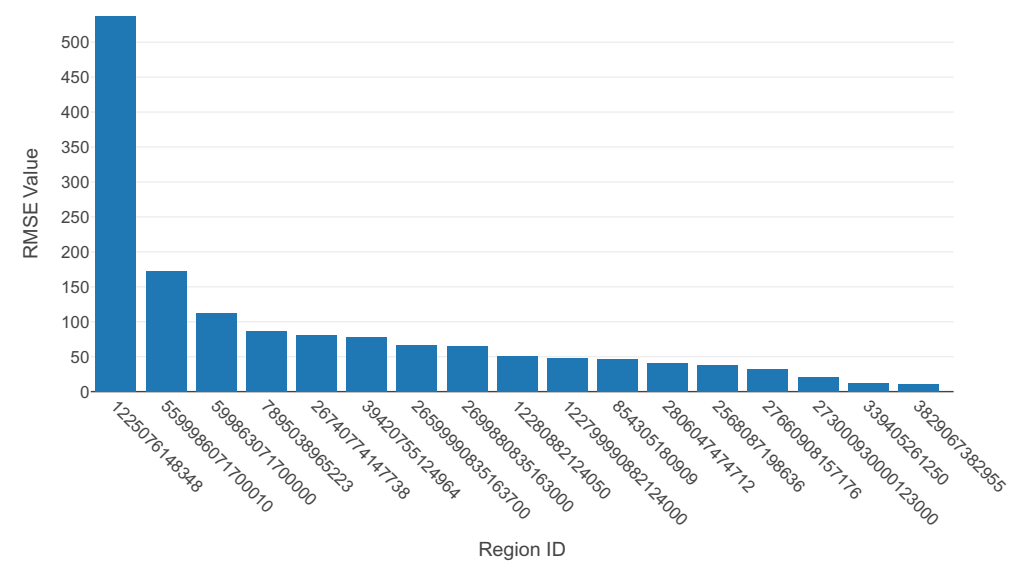

Figure 4.24: RMSE results for predicted trip demand per individual CommercialLarge region. 
Through further inspection of region "1225076148348", we see from Figure 4.25 that our model's predicted outgoing trip request values approximately conform to the fitted line. However, the model over-estimates the prediction values in many high trip volume instances, which causes RMSE values $>2110$ on average. We note that the greatest difference between predicted and actual is during the Afternoon Rush time intervals, indicated on the chart with a green dot, which we conjecture is due to

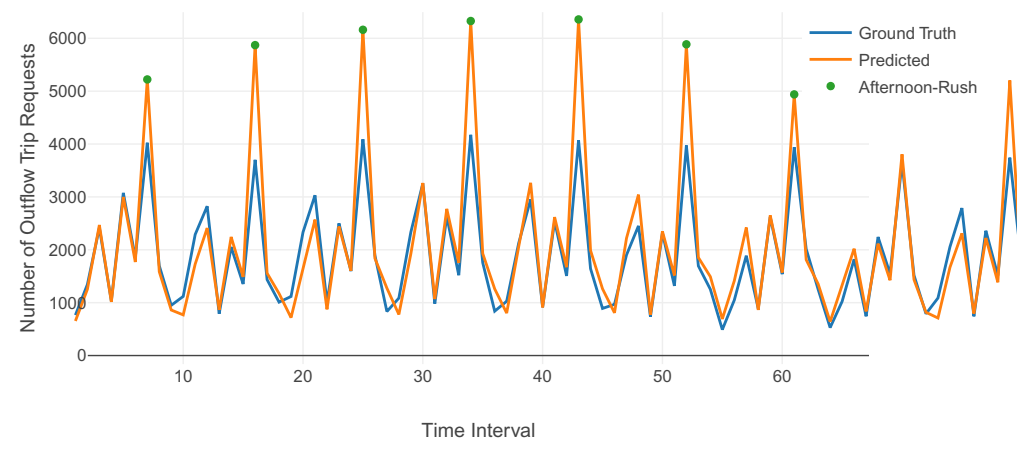

Figure 4.25: Comparison between the predicted total number of trip requests and its respective actual values for region "1225076148348".

the difference between the maximum trip values of the training and testing data (i.e., the training data has a maximum trip value of approximately 4000, while the testing data is much higher). Further, by analyzing the evaluation results by time interval (Figure 4.26), we see that the Afternoon Rush interval results in relatively the highest RMSE error rates, specifically for the Commercial-Large regions (RMSE of 318.3). On the other hand, Morning and Night intervals exhibit relatively the lowest error rates for all regions, which we speculate is due to the low trip volume per region at those interval. 


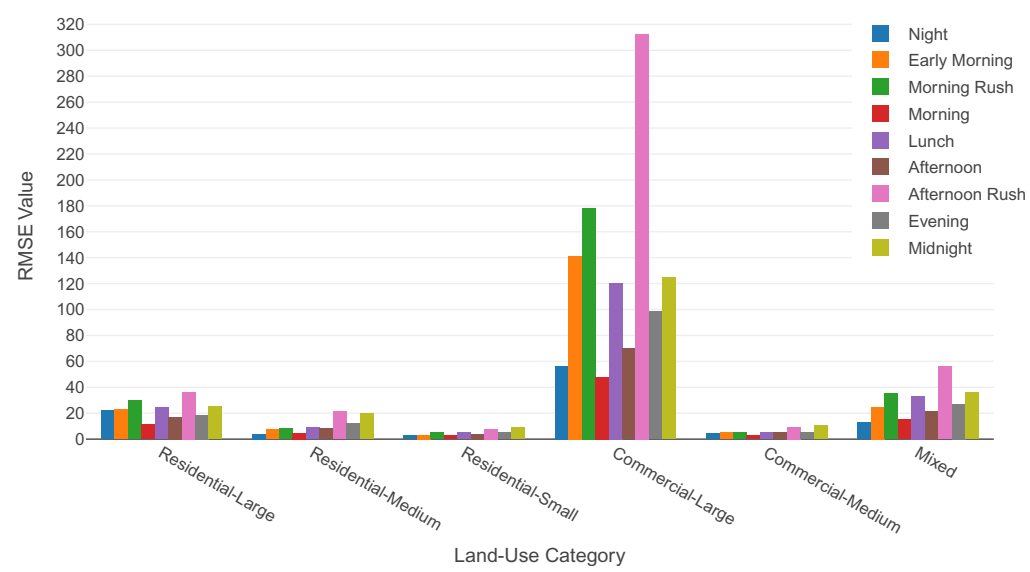

Figure 4.26: RMSE rate for outgoing trip request predictions split by land-use type and colored by time interval (Airport regions were removed due to high error values).

Conversely, we observe that the MSLE results from Figure 4.22a for ResidentialMedium, Residential-Small, and Commercial-Medium are relatively high (0.214, 0.317, and 0.273 respectively), while the RMSE from Figure $4.22 \mathrm{~b}$ are relatively low (15.073, 6.5, and 5.619 respectively). This indicates an inverse situation to that of the Commercial-Large regions. We randomly select a Residential-Medium region (Figure 4.27a) and a Commercial-Medium region (Figure 4.27b) to analyse further. We observe that our model's predictions do not conform to the fitted line and over-estimate its forecasts for most of the relatively low trip volume intervals (in the range of $[0,2]$ outgoing trip requests), resulting in MSLE values $>3.48$ and RMSE values $>5.5$ on average.

From this analysis, we conclude that our node submodel can achieve more reliable localized trip request prediction results for medium and high trip volume regions (regions with an average localized trip request values $>50$ ), albeit with high error rates due to over-estimating some forecast results. However, less reliable results are 


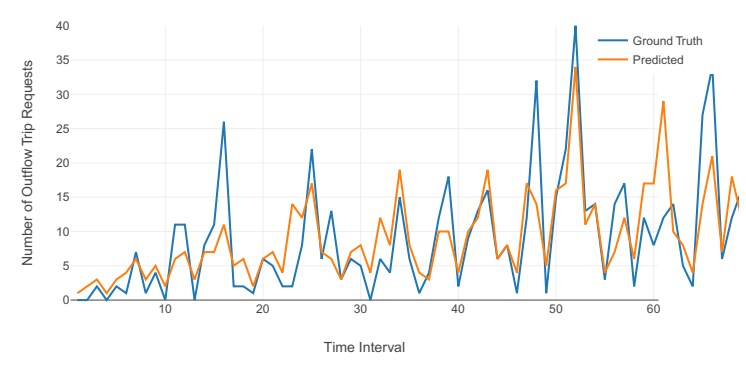

(a) Residential-Medium Region

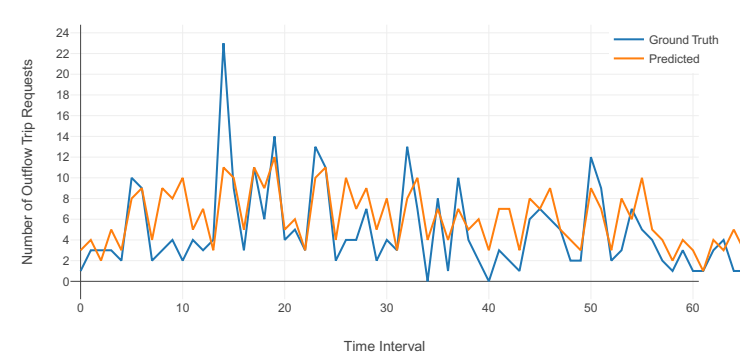

(b) Commercial-Medium Region

Figure 4.27: Comparison between the predicted outgoing trip requests and its respective actual values for two randomly selected regions.

achieved by our model for low trip volume regions (regions with an average localized trip request values $<50)$. We conjecture that medium and high volume regions exhibit periodicity, which can be observed every 9 time intervals on Figure 4.23 and 4.25, allowing our model to learn. However, low volume regions exhibit high volatility within the data preventing our model from learning an accurate representation.

\subsubsection{Edge Submodel's Performance Results per Land-Use Category}

In this section, we discuss the trip demand prediction results by region land-use category on the CityNet- $\omega 36$ model. We note that all statistics are computed per edge as opposed to the previous section, where all calculations were conducted per node. The edge submodel can forecast a close approximation to the global average (Figure 4.28a), standard deviation (Figure 4.28b), and 99 ${ }^{\text {th }}$ percentile (Figure 4.28c) of the ground truth trip demand for each land-use category.

We first analyze the performance by land-use type for origin regions. We observe from Figure 4.29a that the edge submodel produces low MSLE error rates for most 


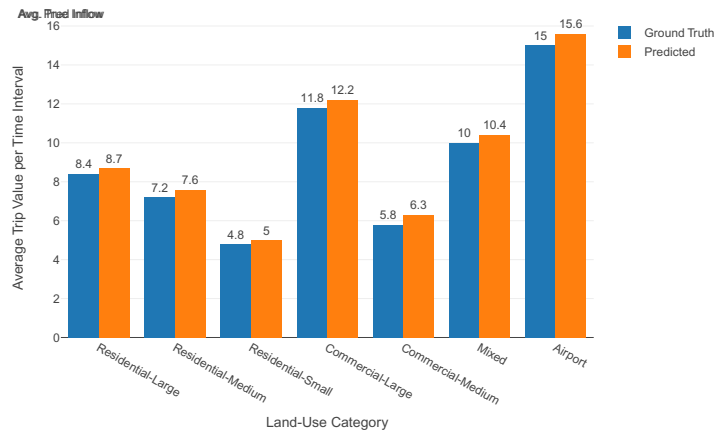

(a) Average

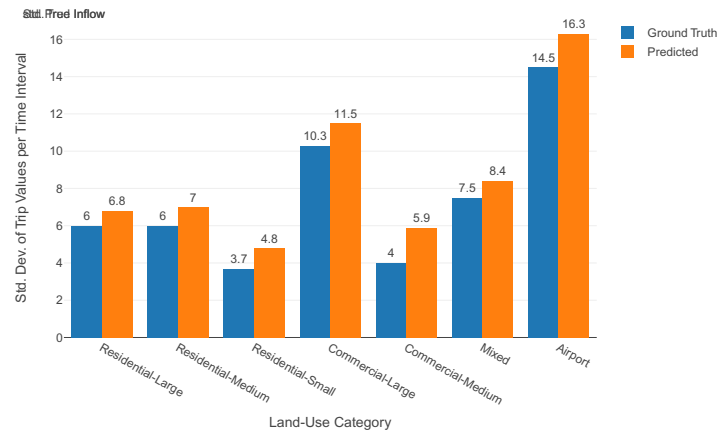

(b) Standard deviation

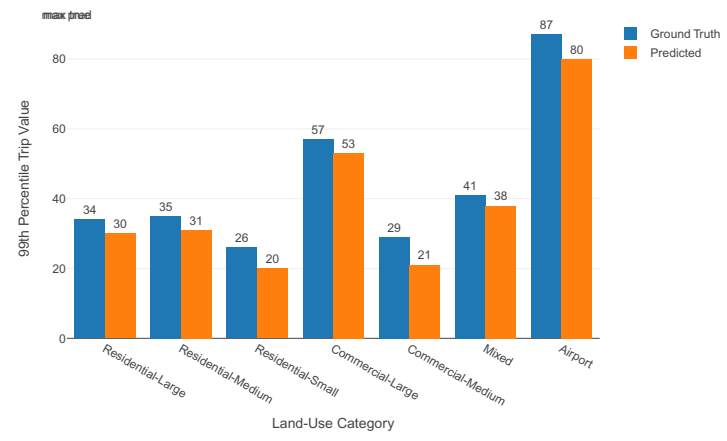

(c) $99^{\text {th }}$ Percentile

Figure 4.28: Average, standard deviation, and $99^{\text {th }}$ Percentile of trip demand by land-use category.

land-use types, except for Residential-Small and Commercial-Medium. We also observe from Figure 4.29b the relatively low RMSE error rates for most regions, except for Commercial-Large and Airport regions, which is the inverse of the MSLE rates. We conjecture that specific regions are the cause of these high error rates. However, for this analysis to be unbiased, we refrain from comparing regions (similar to Figure 4.24), since the number of trip records between all origin-destination region pairs (edges) varies greatly for each time interval, unlike the analysis of the previous section where all regions (nodes) have one record per time interval. 


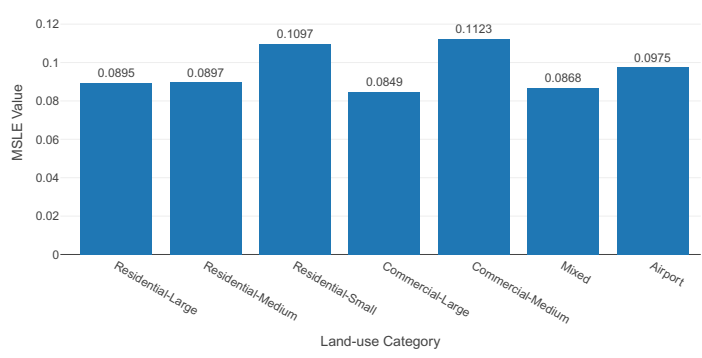

(a) MSLE results

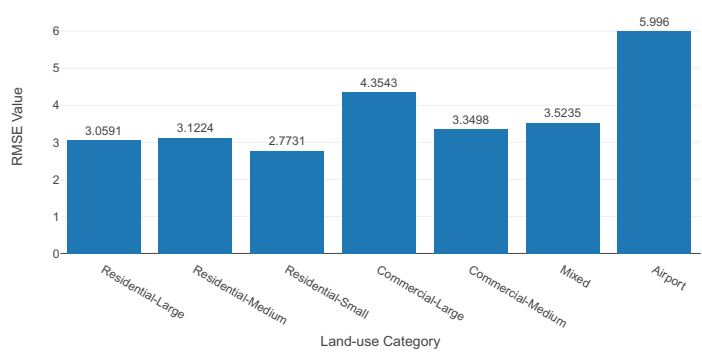

(b) RMSE results

Figure 4.29: Evaluation results by origin region land-use category.

Further, we analyze the performance by land-use type for origin-destination region pairs. This analysis will give us insight into inter-regional correlation. We observe from Figure 4.30 that our model's performance is highly accurate with low MSLE rates $(0.09 \pm 0.018)$ and $\mathrm{RMSE}$ rates $(2.84 \pm 1.68)$ for most region pairs, with larger deviations occurring for Residential-Small and Commercial-Medium regions on the MSLE results chart, and for Airport and Commercial-Large regions on the RMSE results chart. We randomly select a Residential-Large/Commercial-Large region pair

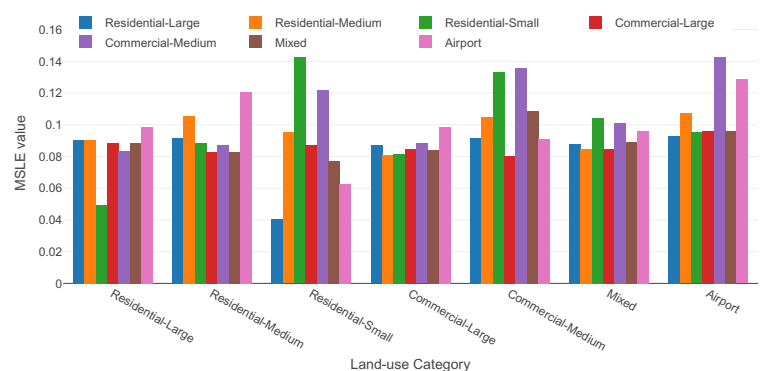

(a) MSLE results

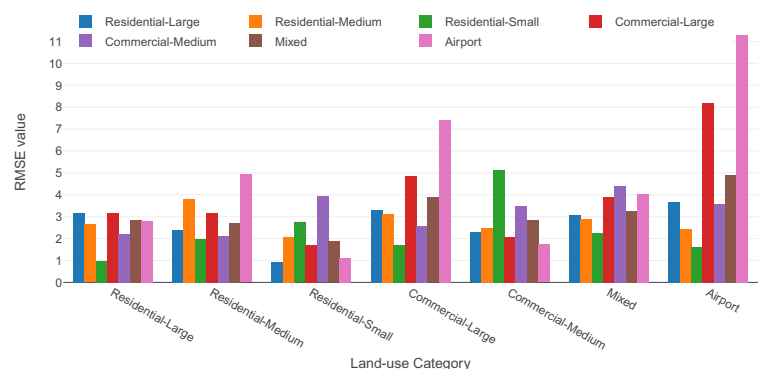

(b) RMSE results

Figure 4.30: Evaluation results by origin region land-use category and by destination region land-use category.

with average error rates for both MSLE and RMSE to illustrate our model's forecast accuracy. We see from Figure 4.31a that our model can achieve predictions close to 
the ground truth from low to high range trip demand values. That said, from Figure 4.31b and 4.31c, where we randomly select a Residential-Small/Commercial-Medium region pair and visa versa, we observe that our model can forecast the low values of trip demand but under-estimates the high values, especially during the Morning Rush interval and Evening interval, respectively. We indicate that this result is not ideal since both are high volume time intervals due to natural, recurring travel patterns, going from home to work and back. Lastly, we see from Figure 4.31d that our model
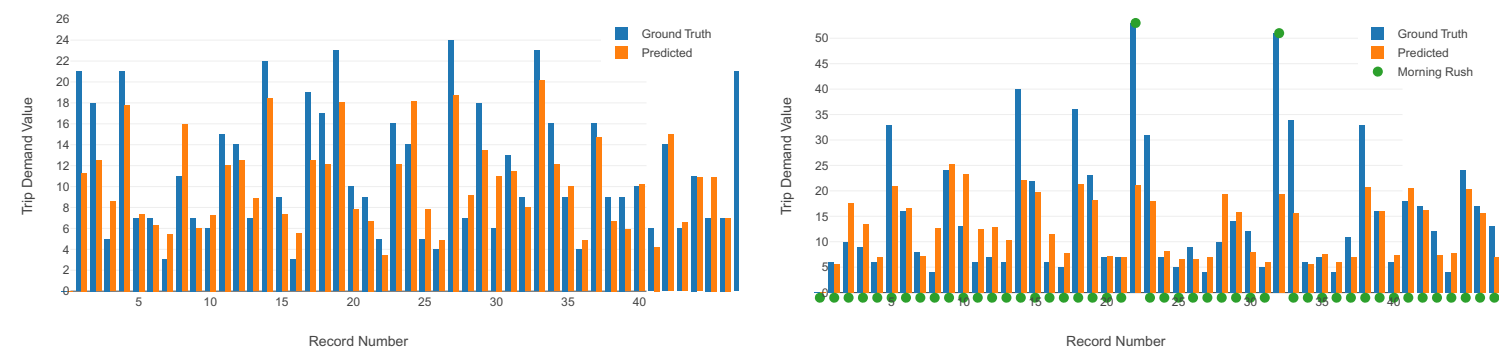

(a) Residential-Large $\rightarrow$ Commercial-Large

(b) Residential-Small $\rightarrow$ Commercial-Medium

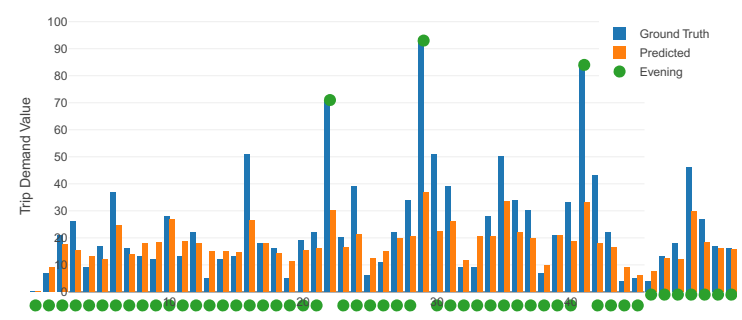

Record Number

(c) Commercial-Medium $\rightarrow$ Residential-Small

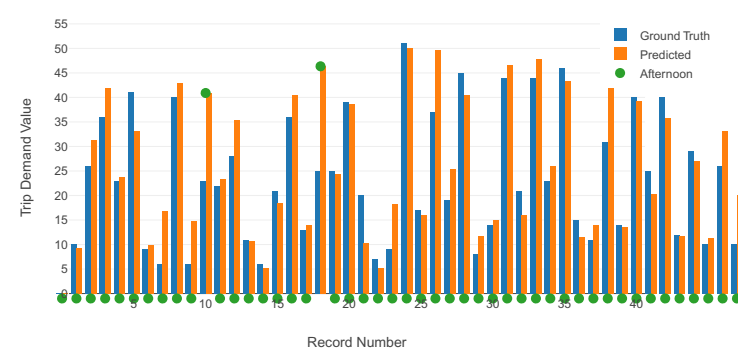

(d) Airport $\rightarrow$ Commercial-Large

Figure 4.31: Comparison between the predicted trip demand and its respective actual values for four randomly selected region pairs by land-use type. Note, we use green dots to indicate over and under estimation for specific time intervals. We also note that we use bar charts since we are depicting separate instances of travel not continues time steps. 
can maintain forecast values close to the ground truth but will over-estimate some instances, especially for the Afternoon time interval causing RMSE vales $>10.67$ on average.

From this analysis, we conclude that our edge submodel can achieve reliable trip demand forecasts for most region pairs. However, less reliable results are achieved between Commercial-Medium and Residential-Small regions, which we conjecture is due to the small number of trip records between them (Figure 4.32) used during the training of the model.

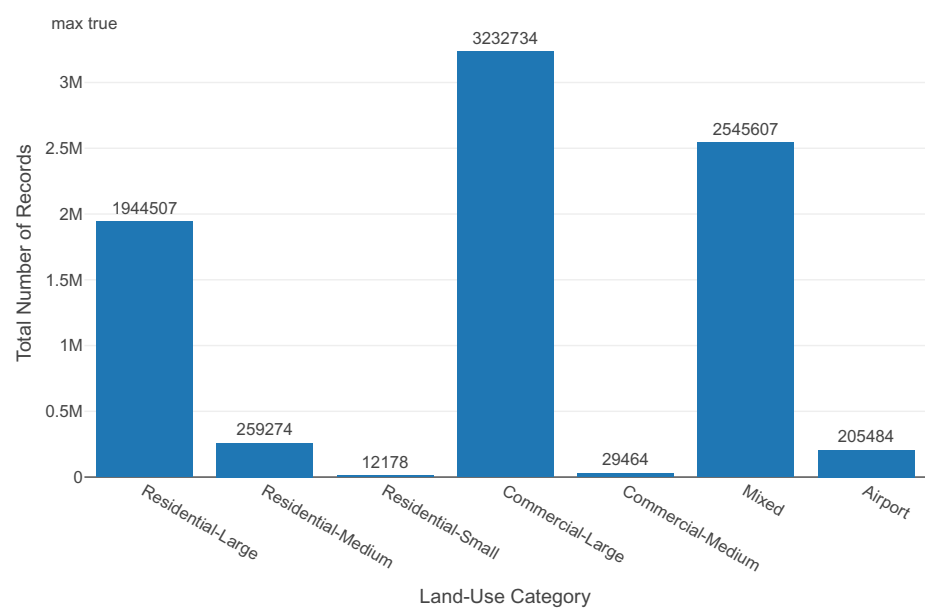

Figure 4.32: Total number of records used by land-use category during the training of the CityNet model. 


\section{Chapter 5}

\section{Conclusions}

In this thesis, we proposed a novel approach for city-wide trip demand forecasting based on past observations of trip records. Compared with existing traffic forecasting approaches, our CityNet model relies on a form of Graph Neural Networks with two distinct computation steps for trip demand prediction:

1. Using Gated Recurrent Units, we capture the temporal dependency of historical trip observations, to produce localized (incoming and outgoing) trip predictions per city region. These localized predictions are then amended to the feature set of their corresponding regions.

2. We engineer feature matrices by associating the pair-wise feature representations of the origin and destination regions, which are then processed by a Convolutional Neural Network to extract patterns from the data elements and infer trip demand predictions. 
Moreover, we evaluated the CityNet model on real-world taxi trip records from the NYC Taxi and Limousine Commission. We found that our proposed model outperforms several forecasting techniques, such as support vector regression, random forest, and gradient tree boosting (XGBoost), as well as several machine learning architectures like the vanilla graph neural network, Wide and Deep neural network, and two variants of our model's implementation. Our findings show that our model can approximate the ground truth with higher accuracy than the compared methods for regions with mid-high traffic volume, while over-estimate for regions with low traffic volume, which is the desired outcome in that case.

Furthermore, from our experiments and work on this research, we found that the socio-economic features and land-use categories, in conjunction with weather condi-

tions, are well suited for trip demand prediction. Additionally, by having a model that has learned abstract feature representations of city regions, not dependent on geographical or spatial constraints, we anticipate that the learned knowledge from one city can be transferred to another with similar socio-economic traits.

We further discuss the benefits and drawback of our approach in Section 5.1, while in Section 5.2 we discuss future work that can help improve the accuracy of our model.

\subsection{Benefits and Limitations}

Our proposed solution to the trip demand forecasting problem benefits from the following:

- Our method does not rely on long-term historical data to generate predictions, 
i.e., it does not maintain a hidden state over a large number of past observations. It, however, depends on a short-term look-back window size and thus can be used intermittently.

- The proposed CityNet model can be utilized to generate city-wide trip demand predictions. For instance, a dispatching system can anticipate future supply and demand levels by predicting the number of incoming and outgoing trip requests for city regions and allocate fleet vehicles ahead of time. Our model can also be used on-demand between a origin and destination region pair independent from other regions, where the input is merely the window of historical observations. This approach makes our model ideal for ride-sharing, and prediction as a service (PaaS) applications. For example, a ride-sharing application can find a route between pickup and drop-off locations and predict future trip demand between all intermediate regions.

- We use a dynamic graph structure to represent a target city without constraining the representation with fixed locations (i.e., raster data or adjacency matrix). This approach allows for nodes and edges to be added and removed dynamically from the graph, which enables our CityNet model to adjust to a growing city with newly added regions or focus the inference on a smaller subset.

- Our proposed method can be applied to any graph-structured data with implicit edge connections, and it can serve time-series forecasting tasks where the prediction on edges benefit from knowing future demand values on the adjacent nodes. We conjecture that the proposed method can be applied to SupplyDemand, Origin-Destination, and link prediction problems. 
Further, our proposed CityNet model and experimental setup suffer from the following limitations:

- From our experiments we found that our model requires a large number of training samples to generate trip demand predication with high fidelity.

- Our experiments are constraint to constant socio-economic features, which is not a reflection of real-world situations.

- The accuracy of the trip demand predictions from the edge submodel (Section 3.3.2) depend on an approximation of the localized trip requests (Section 3.3.1), which indicates that we are missing key features that can better capture the distribution of incoming and outgoing trip requests on the edges.

\section{$5.2 \quad$ Future Work}

For future work, we propose to improve several aspects of our method and its preprocesses:

- We plan to test our CityNet model on the year 2015 trip records and compare the results to the year 2014 .

- We plan to address the large deviations in trip volume per land-use type (displayed in figure 4.11) by investigating granular area partitioning methods that account for complex land-use categorization and don't require a heuristic for consolidation. Additionally, we would like to include detailed land-use types, 
such as transportation hubs (bus and train stations), to achieve more accurate interregional correlations.

- We propose to augment the graph node and edge attribute set with dynamic features, such as region attraction levels or regional events (e.g., ball games) that might explain the surge of trip demand in a region. We also aim to include hourly and more detailed weather information, like sunny or thunderstorm, to explain the fluctuation of trips during the course of a day.

- We would like to incorporate graph attention mechanisms to measure the impact of a region on other connected regions. The intuition is to find better distributions for the computed incoming and outgoing trip requests by measuring the impact of all origin regions on a given destination region and the impact of all destination regions on a given origin regions.

- For our current work, we were only able to reliably retrieve a comprehensive set of information for the city of New York. Thus as future work, we are interested in modeling different cities of varying demographic and human travel patterns. A prominent example, studied in many recent research papers, is the city of Beijing, China. We would also like to test our trained model on cities that share similarities with NYC, i.e., metropolitan cities. 


\section{Bibliography}

[1] J. Zhang, Y. Zheng, and D. Qi, "Deep spatio-temporal residual networks for citywide crowd flows prediction," in Proceedings of the Thirty-First AAAI Conference on Artificial Intelligence, AAAI'17, p. 1655-1661, AAAI Press, 2017.

[2] A. Oord, S. Dieleman, H. Zen, K. Simonyan, O. Vinyals, A. Graves, N. Kalchbrenner, A. Senior, and K. Kavukcuoglu, "Wavenet: A generative model for raw audio," in $S S W, 2016$.

[3] H. Yao, F. Wu, J. Ke, X. Tang, Y. Jia, S. Lu, P. Gong, J. Ye, and Z. Li, "Deep multi-view spatial-temporal network for taxi demand prediction," in AAAI, 2018.

[4] B. Yu, H. Yin, and Z. Zhu, "Spatio-temporal graph convolutional networks: A deep learning framework for traffic forecasting," in Proceedings of the 27th International Joint Conference on Artificial Intelligence, IJCAI'18, p. 3634-3640, AAAI Press, 2018.

[5] Z. Pan, Y. Liang, W. Wang, Y. Yu, Y. Zheng, and J. Zhang, "Urban traffic prediction from spatio-temporal data using deep meta learning," in Proceedings of the 25th ACM SIGKDD International Conference on Knowledge Discovery 8 
Data Mining, KDD '19, p. 1720-1730, Association for Computing Machinery, 2019.

[6] M. S. Ahmed and A. Cook, "Analysis of freeway traffic time-series data by using box-jenkins techniques," Transportation Research Record, 1979.

[7] X. Yin, G. Wu, J. Wei, Y. Shen, H. Qi, and B. Yin, "A comprehensive survey on traffic prediction," ArXiv, vol. abs/2004.08555, 2020.

[8] E. Bolshinsky and R. Freidman, "Traffic flow forecast survey," 2012. Retrieved from: http://www.cs.technion.ac.il/users/wwwb/cgi-bin/trget.cgi/2012/CS/CS-2012-06.pdf. Accessed on March 3 $3^{\text {rd }} 2021$.

[9] E. Vlahogianni, M. G. Karlaftis, and J. C. Golias, "Short-term traffic forecasting: Where we are and where we're going," Transportation Research Part C-emerging Technologies, vol. 43, pp. 3-19, 2014.

[10] S. Guo, Y. Lin, S. Li, Z. Chen, and H. Wan, "Deep spatial-temporal 3d convolutional neural networks for traffic data forecasting," IEEE Transactions on Intelligent Transportation Systems, vol. 20, pp. 3913-3926, 2019.

[11] J. Zhang, Y. Zheng, D. Qi, R. Li, and X. Yi, "Dnn-based prediction model for spatio-temporal data," in Proceedings of the 24th ACM SIGSPATIAL International Conference on Advances in Geographic Information Systems, SIGSPACIAL '16, Association for Computing Machinery, 2016.

[12] S. Fang, Q. Zhang, G. Meng, S. Xiang, and C. Pan, "Gstnet: Global spatialtemporal network for traffic flow prediction," in Proceedings of the Twenty-Eighth 
International Joint Conference on Artificial Intelligence, IJCAI-19, pp. 22862293, International Joint Conferences on Artificial Intelligence Organization, 7 2019.

[13] X. Geng, Y. Li, L. Wang, L. Zhang, Q. Yang, J. Ye, and Y. Liu, "Spatiotemporal multi-graph convolution network for ride-hailing demand forecasting," Proceedings of the AAAI Conference on Artificial Intelligence, vol. 33, pp. 3656-3663, Jul. 2019.

[14] Y. Li, R. Yu, C. Shahabi, and Y. Liu, "Diffusion convolutional recurrent neural network: Data-driven traffic forecasting," arXiv: Learning, 2018.

[15] K. Chen, F. Chen, B. Lai, Z. Jin, Y. Liu, K. Li, L. Wei, P. Wang, Y. Tang, J. Huang, and X. Hua, "Dynamic spatio-temporal graph-based cnns for traffic flow prediction," IEEE Access, vol. 8, pp. 185136-185145, 2020.

[16] Z. Diao, X. Wang, D. Zhang, Y. Liu, K. Xie, and S. He, "Dynamic spatialtemporal graph convolutional neural networks for traffic forecasting," in $A A A I$, 2019.

[17] W. Jiang and J. Luo, "Graph neural network for traffic forecasting: A survey," ArXiv, vol. abs/2101.11174, 2021.

[18] S. Shekhar and B. M. Williams, "Adaptive seasonal time series models for forecasting short-term traffic flow," Transportation Research Record, vol. 2024, pp. $116-125,2007$. 
[19] C. Chen, J. Hu, Q. Meng, and Y. Zhang, "Short-time traffic flow prediction with arima-garch model," 2011 IEEE Intelligent Vehicles Symposium (IV), pp. 607$612,2011$.

[20] A. Abadi, T. Rajabioun, and P. A. Ioannou, "Traffic flow prediction for road transportation networks with limited traffic data," IEEE Transactions on Intelligent Transportation Systems, vol. 16, pp. 653-662, 2015.

[21] Changshui Zhang, Shiliang Sun, and Guoqiang Yu, "A bayesian network approach to time series forecasting of short-term traffic flows," in Proceedings. The 7th International IEEE Conference on Intelligent Transportation Systems (IEEE Cat. No.04TH8749), pp. 216-221, 2004.

[22] S. Sun, C. Zhang, and Y. Zhang, "Traffic flow forecasting using a spatio-temporal bayesian network predictor," ArXiv, vol. abs/1712.08883, 2005.

[23] E. Castillo, J. M. Menéndez, and S. Sánchez-Cambronero, "Predicting traffic flow using bayesian networks," Transportation Research Part B-methodological, vol. 42, pp. 482-509, 2008.

[24] N. Zhang, Y. Zhang, and H. Lu, "Seasonal autoregressive integrated moving average and support vector machine models," Transportation Research Record, vol. 2215 , pp. 85 - 92, 2011.

[25] M. Lippi, M. Bertini, and P. Frasconi, "Short-term traffic flow forecasting: An experimental comparison of time-series analysis and supervised learning," IEEE Transactions on Intelligent Transportation Systems, vol. 14, pp. 871-882, 2013. 
[26] L. Moreira-Matias, J. Gama, M. Ferreira, J. Mendes-Moreira, and L. Damas, "Predicting taxi-passenger demand using streaming data," IEEE Transactions on Intelligent Transportation Systems, vol. 14, pp. 1393-1402, 2013.

[27] M. Voort, M. Dougherty, and S. M. Watson, "Combining kohonen maps with arima time series models to forecast traffic flow," Transportation Research Part C-emerging Technologies, vol. 4, pp. 307-318, 1996.

[28] B. M. Williams and L. Hoel, "Modeling and forecasting vehicular traffic flow as a seasonal arima process: Theoretical basis and empirical results," Journal of Transportation Engineering-asce, vol. 129, pp. 664-672, 2003.

[29] J. Pearl, Bayesian Networks: A Model of Self-activated Memory for Evidential Reasoning. Report, UCLA, Computer Science Department, 1985.

[30] K. H. Pearson, "Vii. note on regression and inheritance in the case of two parents," Proceedings of the Royal Society of London, vol. 58, pp. 240 - 242, 1895.

[31] B. E. Boser, I. M. Guyon, and V. N. Vapnik, "A training algorithm for optimal margin classifiers," in Proceedings of the Fifth Annual Workshop on Computational Learning Theory, COLT '92, p. 144-152, Association for Computing Machinery, 1992.

[32] H. Drucker, C. J. C. Burges, L. Kaufman, A. Smola, and V. Vapnik, "Support vector regression machines," in Proceedings of the 9th International Conference on Neural Information Processing Systems, NIPS'96, p. 155-161, MIT Press, 1996. 
[33] Y. Lv, Y. Duan, W. Kang, Z. Li, and F. Wang, "Traffic flow prediction with big data: A deep learning approach," IEEE Transactions on Intelligent Transportation Systems, vol. 16, pp. 865-873, 2015.

[34] N. Polson and V. Sokolov, "Deep learning for short-term traffic flow prediction," Transportation Research Part C-emerging Technologies, vol. 79, pp. 1-17, 2017.

[35] K. Niu, C. Wang, X. Zhou, and T. Zhou, "Predicting ride-hailing service demand via rpa-lstm," IEEE Transactions on Vehicular Technology, vol. 68, pp. 42134222,2019 .

[36] W. Jin, Y. Lin, Z. Wu, and H. Wan, "Spatio-temporal recurrent convolutional networks for citywide short-term crowd flows prediction," in Proceedings of the 2nd International Conference on Compute and Data Analysis, ICCDA 2018, p. 28-35, Association for Computing Machinery, 2018.

[37] Z. Cui, K. Henrickson, R. Ke, and Y. Wang, "Traffic graph convolutional recurrent neural network: A deep learning framework for network-scale traffic learning and forecasting," IEEE Transactions on Intelligent Transportation Systems, vol. 21, pp. 4883-4894, 2020.

[38] H. Yao, X. Tang, H. Wei, G. Zheng, Y. Yu, and Z. Li, "Modeling spatial-temporal dynamics for traffic prediction," ArXiv, vol. abs/1803.01254, 2018.

[39] Y. LeCun, B. Boser, J. S. Denker, D. Henderson, R. E. Howard, W. Hubbard, and L. D. Jackel, "Backpropagation applied to handwritten zip code recognition," Neural Computation, vol. 1, no. 4, pp. 541-551, 1989. 
[40] A. Krizhevsky, I. Sutskever, and G. E. Hinton, "Imagenet classification with deep convolutional neural networks," Communications of the ACM, vol. 60, pp. $84-$ 90, 2012.

[41] K. Simonyan and A. Zisserman, "Very deep convolutional networks for large-scale image recognition," CoRR, vol. abs/1409.1556, 2015.

[42] C. Szegedy, W. Liu, Y. Jia, P. Sermanet, S. E. Reed, D. Anguelov, D. Erhan, V. Vanhoucke, and A. Rabinovich, "Going deeper with convolutions," 2015 IEEE Conference on Computer Vision and Pattern Recognition (CVPR), pp. 1-9, 2015.

[43] R. B. Girshick, "Fast r-cnn," 2015 IEEE International Conference on Computer Vision (ICCV), pp. 1440-1448, 2015.

[44] J. Redmon, S. Divvala, R. B. Girshick, and A. Farhadi, "You only look once: Unified, real-time object detection," 2016 IEEE Conference on Computer Vision and Pattern Recognition (CVPR), pp. 779-788, 2016.

[45] P. Sermanet, D. Eigen, X. Zhang, M. Mathieu, R. Fergus, and Y. LeCun, "Overfeat: Integrated recognition, localization and detection using convolutional networks," CoRR, vol. abs/1312.6229, 2014.

[46] W. Jin, Y. Lin, Z. Wu, and H. Wan, "Spatio-temporal recurrent convolutional networks for citywide short-term crowd flows prediction," in Proceedings of the 2nd International Conference on Compute and Data Analysis, ICCDA 2018, p. 28-35, Association for Computing Machinery, 2018. 
[47] V. Pham, C. Kermorvant, and J. Louradour, "Dropout improves recurrent neural networks for handwriting recognition," 201414 th International Conference on Frontiers in Handwriting Recognition, pp. 285-290, 2014.

[48] A. Graves, "Generating sequences with recurrent neural networks," ArXiv, vol. abs/1308.0850, 2013.

[49] W. Zaremba, I. Sutskever, and O. Vinyals, "Recurrent neural network regularization," ArXiv, vol. abs/1409.2329, 2014.

[50] H. Sak, A. Senior, and F. Beaufays, "Long short-term memory based recurrent neural network architectures for large vocabulary speech recognition," ArXiv, vol. abs/1402.1128, 2014.

[51] Y. Fan, Y. Qian, F. Xie, and F. Soong, "Tts synthesis with bidirectional lstm based recurrent neural networks," in INTERSPEECH, pp. 1964-1968, 2014.

[52] K. Cho, B. V. Merrienboer, Çaglar Gülçehre, D. Bahdanau, F. Bougares, H. Schwenk, and Y. Bengio, "Learning phrase representations using rnn encoderdecoder for statistical machine translation," ArXiv, vol. abs/1406.1078, 2014.

[53] J. Donahue, L. A. Hendricks, M. Rohrbach, S. Venugopalan, S. Guadarrama, K. Saenko, and T. Darrell, "Long-term recurrent convolutional networks for visual recognition and description," 2015 IEEE Conference on Computer Vision and Pattern Recognition (CVPR), pp. 2625-2634, 2015.

[54] E. Marchi, G. Ferroni, F. Eyben, L. Gabrielli, S. Squartini, and B. Schuller, "Multi-resolution linear prediction based features for audio onset detection with 
bidirectional lstm neural networks," 2014 IEEE International Conference on Acoustics, Speech and Signal Processing (ICASSP), pp. 2164-2168, 2014.

[55] S. K. Sønderby and O. Winther, "Protein secondary structure prediction with long short term memory networks," ArXiv, vol. abs/1412.7828, 2014.

[56] B. Liao, J. Zhang, C. Wu, D. McIlwraith, T. Chen, S. Yang, Y. Guo, and F. Wu, "Deep sequence learning with auxiliary information for traffic prediction," in Proceedings of the 24th ACM SIGKDD International Conference on Knowledge Discovery \& Data Mining, KDD '18, p. 537-546, Association for Computing Machinery, 2018.

[57] D. Zhang and M. Kabuka, "Combining weather condition data to predict traffic flow: A gru based deep learning approach," 2017 IEEE 15th Intl Conf on Dependable, Autonomic and Secure Computing, 15th Intl Conf on Pervasive Intelligence and Computing, 3rd Intl Conf on Big Data Intelligence and Computing and Cyber Science and Technology Congress(DASC/PiCom/DataCom/CyberSciTech), pp. 1216-1219, 2017.

[58] S. Hochreiter and J. Schmidhuber, "Long short-term memory," Neural Computation, vol. 9, pp. 1735-1780, 1997.

[59] J. Bruna, W. Zaremba, A. Szlam, and Y. LeCun, "Spectral networks and locally connected networks on graphs," CoRR, vol. abs/1312.6203, 2014.

[60] M. Defferrard, X. Bresson, and P. Vandergheynst, "Convolutional neural networks on graphs with fast localized spectral filtering," in Proceedings of the 30th 
International Conference on Neural Information Processing Systems, NIPS'16, (Red Hook, NY, USA), p. 3844-3852, Curran Associates Inc., 2016.

[61] T. N. Kipf and M. Welling, "Semi-supervised classification with graph convolutional networks," 2017.

[62] M. Niepert, M. Ahmed, and K. Kutzkov, "Learning convolutional neural networks for graphs," in Proceedings of the 33rd International Conference on International Conference on Machine Learning - Volume 48, ICML'16, p. 2014-2023, JMLR.org, 2016.

[63] J. Atwood and D. Towsley, "Diffusion-convolutional neural networks," in NIPS, 2016.

[64] Y. Li, Z. Zhu, D. Kong, M. Xu, and Y. Zhao, "Learning heterogeneous spatialtemporal representation for bike-sharing demand prediction," Proceedings of the AAAI Conference on Artificial Intelligence, vol. 33, pp. 1004-1011, Jul. 2019.

[65] P. Velickovic, G. Cucurull, A. Casanova, A. Romero, P. Liò, and Y. Bengio, "Graph attention networks," ArXiv, vol. abs/1710.10903, 2018.

[66] J. Chung, Çaglar Gülçehre, K. Cho, and Y. Bengio, "Empirical evaluation of gated recurrent neural networks on sequence modeling," ArXiv, vol. abs/1412.3555, 2014.

[67] W. Yin, K. Kann, M. Yu, and H. Schütze, "Comparative study of cnn and rnn for natural language processing," ArXiv, vol. abs/1702.01923, 2017.

[68] X. Glorot and Y. Bengio, "Understanding the difficulty of training deep feedforward neural networks," in Proceedings of the Thirteenth International Conference 
on Artificial Intelligence and Statistics, vol. 9 of Proceedings of Machine Learning Research, pp. 249-256, JMLR Workshop and Conference Proceedings, 2010.

[69] M. Abadi, A. Agarwal, P. Barham, E. Brevdo, Z. Chen, C. Citro, G. S. Corrado, A. Davis, J. Dean, M. Devin, S. Ghemawat, I. Goodfellow, A. Harp, G. Irving, M. Isard, Y. Jia, R. Jozefowicz, L. Kaiser, M. Kudlur, J. Levenberg, D. Mané, R. Monga, S. Moore, D. Murray, C. Olah, M. Schuster, J. Shlens, B. Steiner, I. Sutskever, K. Talwar, P. Tucker, V. Vanhoucke, V. Vasudevan, F. Viégas, O. Vinyals, P. Warden, M. Wattenberg, M. Wicke, Y. Yu, and X. Zheng, "TensorFlow: Large-scale machine learning on heterogeneous systems," 2015. Software available from tensorflow.org. Accessed on March $3^{\text {rd }}, 2021$.

[70] F. Chollet et al., "Keras," 2015. Software available from keras.io. Accessed on March $3^{r d}, 2021$.

[71] P. Battaglia, J. B. Hamrick, V. Bapst, A. Sanchez-Gonzalez, V. Zambaldi, M. Malinowski, A. Tacchetti, D. Raposo, A. Santoro, R. Faulkner, Çaglar Gülçehre, H. Song, A. Ballard, J. Gilmer, G. Dahl, A. Vaswani, K. R. Allen, C. Nash, V. Langston, C. Dyer, N. Heess, D. Wierstra, P. Kohli, M. Botvinick, O. Vinyals, Y. Li, and R. Pascanu, "Relational inductive biases, deep learning, and graph networks," ArXiv, vol. abs/1806.01261, 2018.

[72] D. Wang, W. Cao, J. Li, and J. Ye, "Deepsd: Supply-demand prediction for online car-hailing services using deep neural networks," 2017 IEEE 33rd International Conference on Data Engineering (ICDE), pp. 243-254, 2017.

[73] T. Chen and C. Guestrin, "Xgboost: A scalable tree boosting system," in Proceedings of the 22nd ACM SIGKDD International Conference on Knowledge 
Discovery and Data Mining, KDD '16, p. 785-794, Association for Computing Machinery, 2016.

[74] H.-T. Cheng, L. Koc, J. Harmsen, T. Shaked, T. Chandra, H. Aradhye, G. Anderson, G. Corrado, W. Chai, M. Ispir, R. Anil, Z. Haque, L. Hong, V. Jain, X. Liu, and H. Shah, "Wide \& deep learning for recommender systems," in Proceedings of the 1st Workshop on Deep Learning for Recommender Systems, DLRS 2016, p. 7-10, Association for Computing Machinery, 2016.

[75] M. Löning, A. J. Bagnall, S. Ganesh, V. Kazakov, J. Lines, and F. Király, "sktime: A unified interface for machine learning with time series," ArXiv, vol. abs/1909.07872, 2019.

[76] I. Kanellopoulos and G. Wilkinson, "Strategies and best practice for neural network image classification," International Journal of Remote Sensing, vol. 18, pp. 711-725, 1997.

[77] Y. LeCun, L. Bottou, G. Orr, and K. Müller, Efficient BackProp, pp. 9-48. Berlin, Heidelberg: Springer Berlin Heidelberg, 2012.

[78] R. D. Kleinberg, Y. Li, and Y. Yuan, "An alternative view: When does sgd escape local minima?," ArXiv, vol. abs/1802.06175, 2018.

[79] K. You, M. Long, and M. I. Jordan, "How does learning rate decay help modern neural networks," arXiv: Learning, 2019.

[80] I. Sutskever, J. Martens, G. Dahl, and G. Hinton, "On the importance of initialization and momentum in deep learning," in Proceedings of the 30th International 
Conference on Machine Learning, vol. 28 of Proceedings of Machine Learning Research, pp. 1139-1147, PMLR, 2013.

[81] R. Caruana, S. Lawrence, and C. Giles, "Overfitting in neural nets: Backpropagation, conjugate gradient, and early stopping.," in Advances in Neural Information Processing Systems, vol. 13, pp. 402-408, 2000.

[82] I. Goodfellow, Y. Bengio, and A. Courville, Deep Learning. The MIT Press, 2016.

[83] M. Li, M. Soltanolkotabi, and S. Oymak, "Gradient descent with early stopping is provably robust to label noise for overparameterized neural networks," ArXiv, vol. abs/1903.11680, 2020.

[84] J. Ke, X. Qin, H. Yang, Z. Zheng, Z. Zhu, and J. Ye, "Predicting origindestination ride-sourcing demand with a spatio-temporal encoder-decoder residual multi-graph convolutional network," ArXiv, vol. abs/1910.09103, 2019. 


\section{Appendix A}

\section{Land-Use Labeling Heuristic}

In Section 4.1.1.2 we described the land-use classification procedure that was used to classify city regions with land-use types. One step of the classification process is a heuristic function that labels each census tract with a single land-use type based on 1-or-more Zoning and Land-Use (ZoLa) data fields. In the following sections, we define preliminaries, followed by the heuristic algorithm.

\section{Preliminaries}

The New York City (NYC) Zoning and Land-Use regulations divide how land is used into four categories, from which we extract specific markers as follows:

1. Resident Districts: they are divided into 10 resolutions based on building size and residential density, which we denote by $R 1-R 10$.

2. Commercial Districts: they are divided into 8 subcategories based on functional 
similarities and locational requirements, which we denote as $C 1-C 8$.

3. Manufacturing District: we denote them by $M A N$.

4. Special Purpose Districts: this category includes special district types that address unique conditions. For our purposes, we extract the following:

(a) Planned Community Preservation Districts: We designate these districts as Residential and denote them by $P C$.

(b) Enhanced Commercial Districts: We designate these districts as Commercial and denote them by $E C$.

(c) Scenic View Districts, Sheepshead Bay District, Natural Area Districts, Parks, and Playgrounds: We designate these districts as Park and denote them as PARK.

(d) Mixed Use Districts, as well as other districts in this category: We designate these districts as Mixed and denote them as $M X$.

We also use the NYC Neighbourhood Tabulation Area (NTA) names to specifically extract Airport and Cemetery regions. In addition, we use the total population in the area obtained from census tract datasets, denoted by pop.

\section{Heuristic Algorithm}

The heuristic is performed for each census tract in the city. It takes as input an array of ZoLa data fields (i.e., a list of the extracted markers), as well as the total population of the tract and its associated NTA name. The algorithm counts the 
number of markers, specific to each class mentioned in Section 4.1.1.2, and then it returns the class based on precedence, which is assigned to the census tract. We detail the algorithm as follows:

Algorithm A.1 Heuristic to label a census tract with a land-use type

Input: Array of ZoLa data fields, L; NTA name, nta; Total Population, pop. Output: Land-use type.

1: Let $r s, r m$, and $r l$ be a counter for Residential small, medium, and large regions, respectively.

2: Let $\mathrm{cm}$ and $\mathrm{cl}$ be a counter for Commercial medium and large regions, respectively.

3: Let mix, $m n$, and $p n$ be a counter for Mixed, Manufacturing, and Park regions, respectively.

4: for all $l \in L$ do

5: $\quad$ if $l$ in $R 1-R 5$ OR $l==P C$ then

6: $\quad r s++$

7: $\quad$ else if $l$ in $R 6-R 7$ then

8: $\quad r m++$

9: $\quad$ else if $l$ in $R 8-R 10$ then

10: $\quad r l++$

11: $\quad$ else if $l$ in $C 1-C 3$ OR $l==E C$ then

12: $\quad \operatorname{mix}++$

13: $\quad$ else if $l==C 4 \mathrm{OR} l$ in $C 7-C 8$ then

14: $\quad \mathrm{cm}++$

15: $\quad$ else if $l$ in $C 5-C 6$ then

16: $\quad c l++$

17: $\quad$ else if $l==M X$ then

18: $\quad \operatorname{mix}++$

19: $\quad$ else if $l==M A N$ then

20: $\quad m n++$

21: $\quad$ else if $l==P A R K$ then

22: $\quad$ pn ++

23: $\quad$ end if

24: end for 


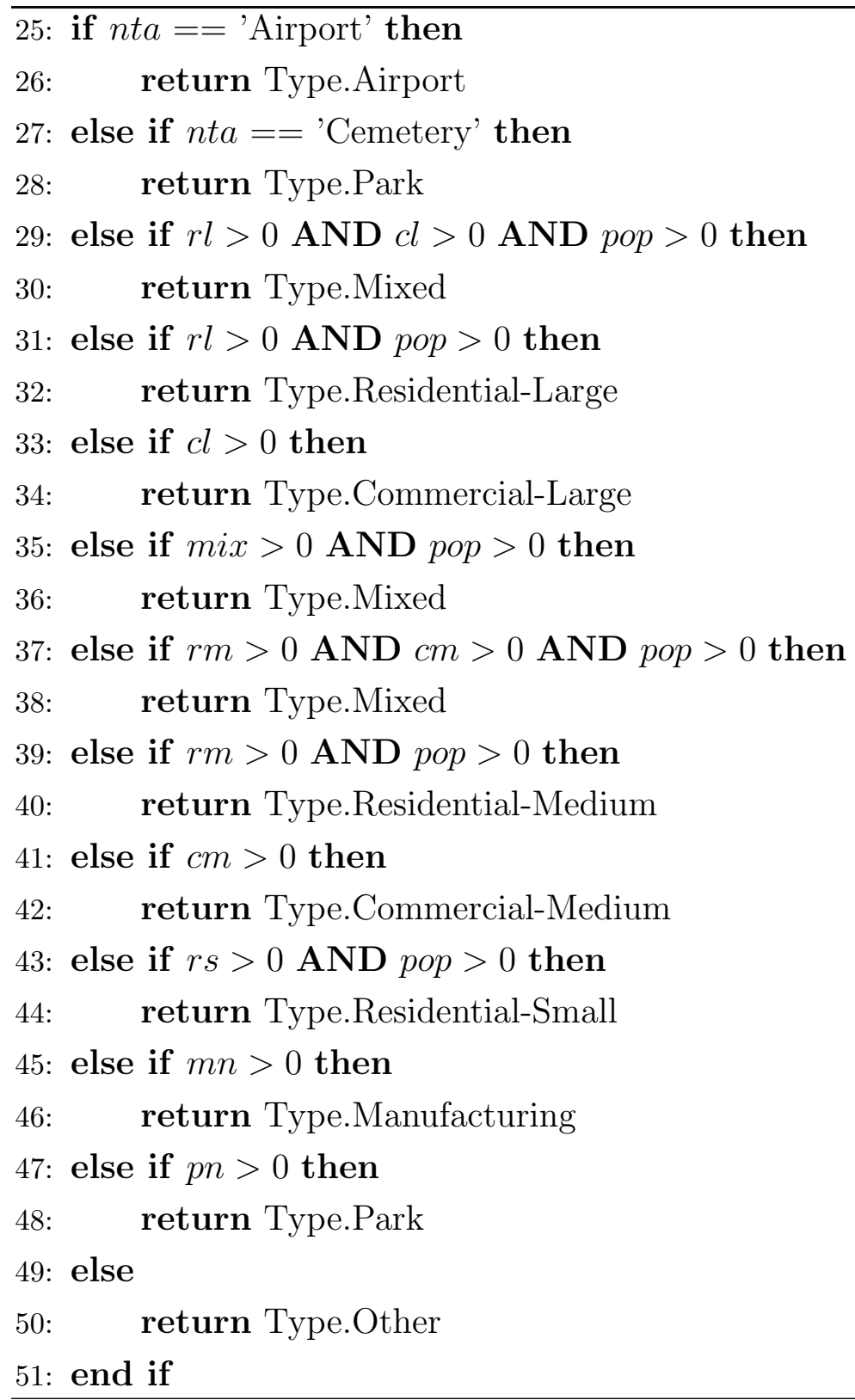

